Supporting Information

\title{
Triptycenyl Sulfide: A Practical and Active Catalyst for Electrophilic Aromatic Halogenation using $\mathbf{N}$-Halosuccinimides
}

\author{
Yuji Nishii,* Mitsuhiro Ikeda, Yoshihiro Hayashi, Susumu Kawauchi, and Masahiro Miura* \\ y_nishii@chem.eng.osaka-u.ac.jp \\ miura@chem.eng.osaka-u.ac.jp
}

\section{Contents}

1. General Information $\quad$ S2

2. Additional Data for the Reaction Condition Screening $\quad$ S3

3. Experimental Procedures and Identification Data

3-1. Preparation of Trip-SMe $\quad$ S6

3-2. Preparation of Trip-OMe $\quad$ S6

3-3. Multi-halogenation of naphthalene (Scheme 2) S6

3-4. Bromination of BINOL (Scheme 3) S8

3-5. General procedure for the Trip-SMe-catalyzed halogenation (Scheme 4, 5) $\quad$ S10

3-6. Bromination of strychnine (strychnidin-10-one) (Scheme 5) S17

3-7. Bromination of Trip-SMe (Scheme 6) S20

3-8. Preparation of [Trip-SMeBr] $\left[\mathrm{SbCl}_{5} \mathrm{Br}\right]_{2}$ (Scheme 8) $\quad \mathrm{S} 21$

$\begin{array}{ll}\text { 4. X-ray Crystallography } & \text { S23 }\end{array}$

5. Kinetic Study

$\begin{array}{ll}5-1 . \text { Time course experiments } & \text { S29 }\end{array}$

5-2. Variable time normalization analysis $\quad$ S32

$\begin{array}{ll}5-3 . & \text { Equilibrium constant measurement } \\ \mathrm{S} 34\end{array}$

6. Copy of NMR Spectra $\quad$ S35

$\begin{array}{lr}\text { 7. References } & \text { S69 }\end{array}$ 


\section{General Information}

All manipulations were performed under $\mathrm{N}_{2}$ using standard Schlenk techniques unless otherwise noted. $\mathrm{MeCN}$ and DMF were dried and deoxygenated by a Glass Counter Solvent Dispending System (Nikko Hansen \& Co., Ltd.). DCE (1,2-dicloroethane) was distillated from $\mathrm{CaH}_{2}$ and stored with molecular sieves 4A. DCM (dichloromethane) was degassed with $\mathrm{N}_{2}$ bubbling and dried over molecular sieves 4A. THF were purchased as dehydrated solvent and used as received. Thioethers ( ${ }^{n}$ Octyl-SMe, 1-Ad-SMe, $\mathrm{Ph}_{3} \mathrm{C}-$ $\mathrm{SMe}$ ) were prepared by standard alkylation of the corresponding thiols treated with iodomethane and $\mathrm{K}_{2} \mathrm{CO}_{3}$ in DMF. BDSB ([Et $\left.\left.{ }_{2} \mathrm{SBr}\right]\left[\mathrm{SbCl}_{5} \mathrm{Br}\right]\right)$ was prepared according to the literature procedure. ${ }^{1}$

Nuclear magnetic resonance spectra were measured at $400 \mathrm{MHz}\left({ }^{1} \mathrm{H} \mathrm{NMR}\right)$ and at $100 \mathrm{MHz}\left({ }^{13} \mathrm{C} \mathrm{NMR}\right)$ in $5 \mathrm{~mm}$ NMR tubes. ${ }^{1} \mathrm{H}$ NMR chemical shifts were reported in ppm relative to the resonance of TMS $(\delta 0.00)$ or the residual solvent signals at $\delta 7.26$ for $\mathrm{CDCl}_{3}$ and at $\delta 5.32$ for $\mathrm{CD}_{2} \mathrm{Cl}_{2} \cdot{ }^{13} \mathrm{C}$ NMR chemical shifts were reported in ppm relative to the residual solvent signals at $\delta 77.2$ for $\mathrm{CDCl}_{3}$ and at $\delta 53.8$ for $\mathrm{CD}_{2} \mathrm{Cl}_{2}$. Melting points were measured with Mettler Toledo MP90. High resolution mass spectra (HRMS) were recorded by EI or APCI-TOF. GC analyses were carried out with Shimadzu GC8APF equipped with a silicon OV-17 column $(2.6 \mathrm{~mm} \times 1.5 \mathrm{~m})$. GC-MS analyses were carried out with Shimadzu GCMS-QP2010 SE equipped with a CBP-1 column $(0.5 \mathrm{~mm} \times 25 \mathrm{~m})$. HPLC analyses were carried out with JASCO EXTREMA (PU4180/MD4015/CO4065) equipped with an YMC CHIRAL ART Cellulose-SB column. Shimadzu GCMSQP2010 SE. Silica gel column chromatography was performed using Wakosil ${ }^{\circledR}$ C-200 (64-210 $\left.\mu \mathrm{m}\right)$. Preparative gel permeation chromatography (GPC) was conducted with Showa Denko H-2001, H-2002 column (eluent: $\mathrm{CHCl}_{3}$ ) or YMC T2000 (eluent: EtOAc) column.

All calculations were carried out using the Gaussian 16 program. ${ }^{2}$ The DFT calculations were carried out using the long-range and dispersion-corrected $\omega$ B97X-D functional. ${ }^{3}$ The 6-311G(d,p) basis set was used for all atoms. ${ }^{4}$ The solvent effect of 1,2-dichloroethane was taken into account by the Polarizable Continuum Model using the integral equation formalism (IEFPCM) ${ }^{5}$ for DFT calculations. The optimized molecular structures were verified by vibrational analysis; equilibrium structures did not have imaginary frequencies. NPA charge was evaluated by the NBO 6.0 program. $^{6}$ 


\section{Additional Data for the Reaction Condition Screening}

Table S1. Optimization study ${ }^{a}$

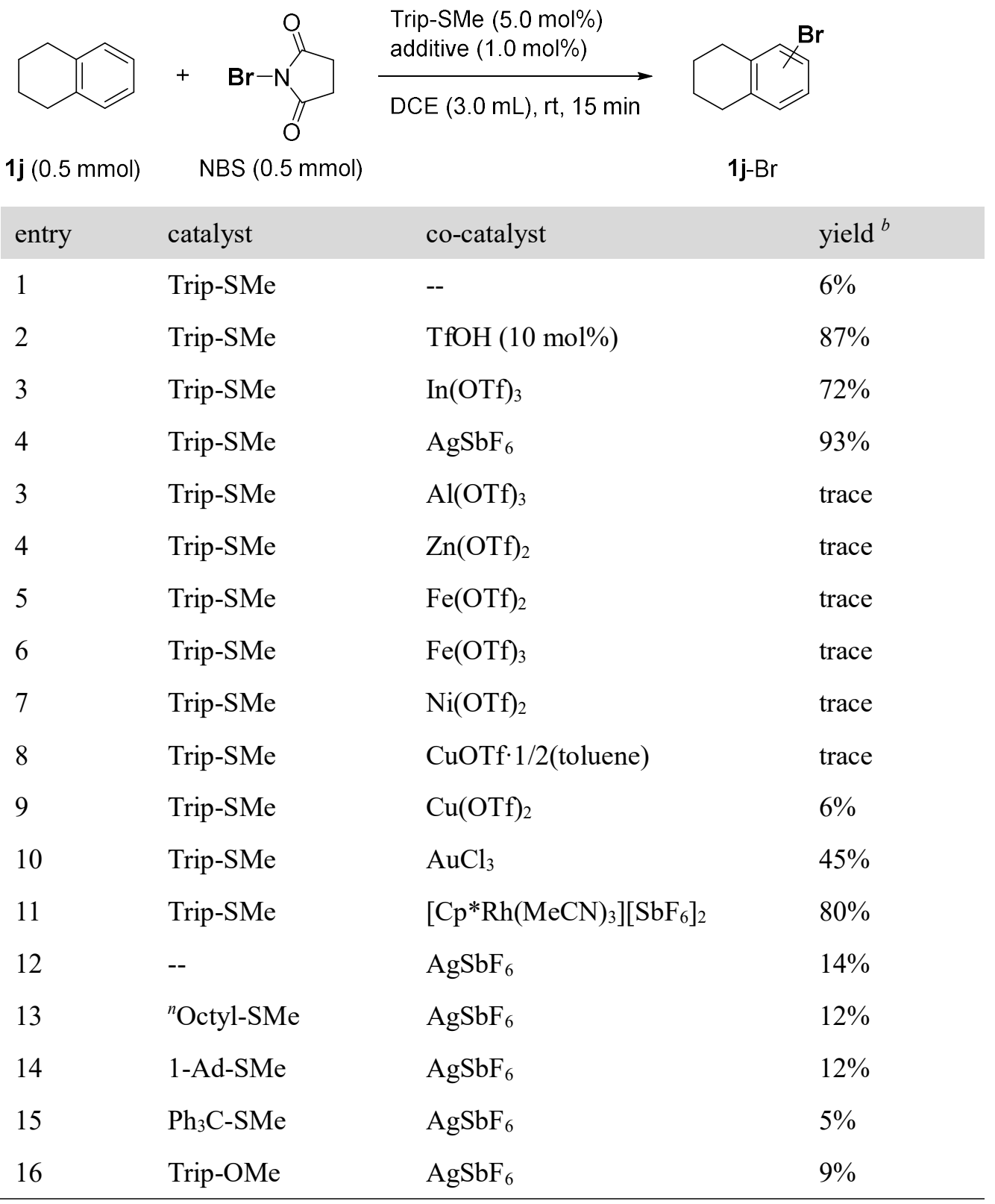

${ }^{a}$ Reaction conditions: 1a $(0.5 \mathrm{mmol}), \mathrm{NBS}(0.5 \mathrm{mmol}), \mathrm{DCE}(3.0 \mathrm{~mL})$.

${ }^{b}$ Combined yield of regioisomers determined by GC analysis. 
Table S2. Bromination and chlorination of 1a with NXS using various catalysts ${ }^{a}$

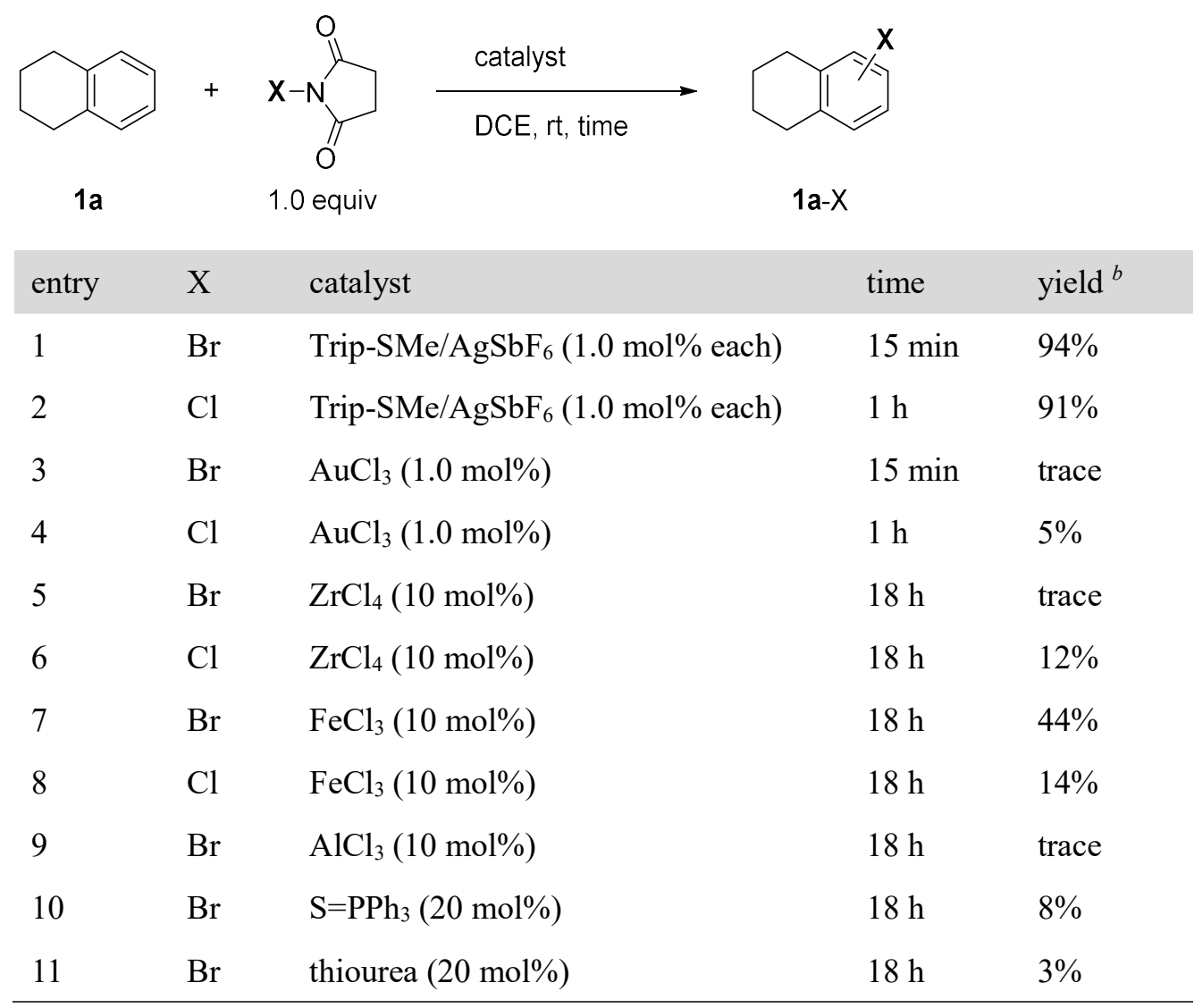

${ }^{a}$ Reaction conditions: 1a $(0.5 \mathrm{mmol})$, NXS $(0.5 \mathrm{mmol})$, DCE $(3.0 \mathrm{~mL})$, Trip-SMe (5.0 mol\%), $\mathrm{AgSbF}_{6}(1.0 \mathrm{~mol} \%)$.

${ }^{b}$ Combined yield of regioisomers determined by GC analysis. 
Scheme S1. Multi-halogenation of napthalene.

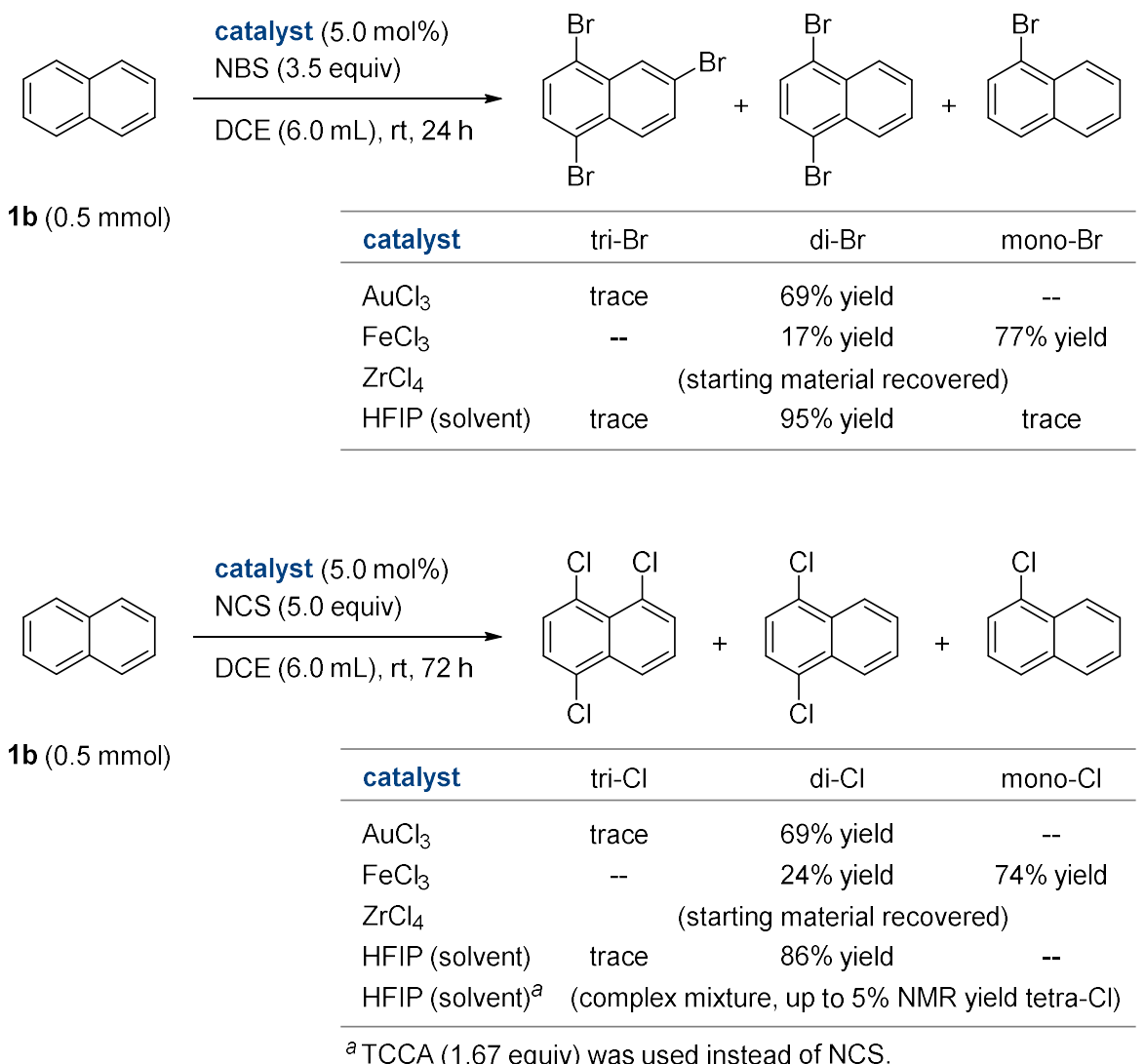

Scheme S2. Bromination of BINOL.<smiles>Oc1ccc2ccccc2c1-c1c(O)ccc2ccccc12</smiles>

1c, $>99 \%$ ee<smiles>O=C1CCC(=O)N1Br</smiles>

catalyst

DCE $(4.0 \mathrm{~mL}), \mathrm{rt}, 24 \mathrm{~h}$

NBS (2.6 equiv)<smiles>Oc1ccc2ccccc2c1-c1c(O)ccc2cc(Br)ccc12</smiles>

1c- $-\mathrm{Br}_{2}$<smiles>Oc1ccc2cc(Br)ccc2c1-c1c(O)ccc2cc(Br)ccc12</smiles>

1c- $-\mathrm{Br}_{2}$

\begin{tabular}{lccc}
\hline catalyst & 1c & $1 \mathrm{c}-\mathrm{Br}$ & $1 \mathrm{c}-\mathrm{Br}_{2}$ \\
\hline $\mathrm{AuCl}_{3}(1.0 \mathrm{~mol} \%)$ & $7 \%$ yield & $23 \%$ yield & $11 \%$ yield \\
$\mathrm{FeCl}_{3}(10 \mathrm{~mol} \%)$ & trace & $10 \%$ yield & $53 \%$ yield \\
$\mathrm{S}=\mathrm{PPh}_{3}(20 \mathrm{~mol} \%)$ & $29 \%$ yield & $28 \%$ yield & $7 \%$ yield \\
thiourea $(20 \mathrm{~mol} \%)$ & $14 \%$ yield & $23 \%$ yield & $6 \%$ yield \\
$\mathrm{BDSB}(5.0 \mathrm{~mol} \%)$ & $8 \%$ yield & $20 \%$ yield & $7 \%$ yield \\
$\mathrm{HFIP}$ (solvent) & $43 \%$ yeld & trace & $10 \%$ yield \\
\hline
\end{tabular}




\section{Experimental Procedures and Identification Data}

\section{3-1. Preparation of Trip-SMe}

A two-neck round-bottom flask equipped with an $\mathrm{N}_{2}$ balloon and a rubber cap was charged with 9bromotriptycene $(1.39 \mathrm{~g}, 4.17 \mathrm{mmol})$. THF $(30 \mathrm{~mL})$ was added via syringe and the mixture was stirred at room temperature to dissolve the solid. ${ }^{n} \mathrm{BuLi}(1.55 \mathrm{M}$ in hexane, $5.0 \mathrm{mmol})$ was added dropwise at $-78{ }^{\circ} \mathrm{C}$, and the resulting suspension was stirred at this temperature for $1 \mathrm{~h}$. Dimethyldisulfide (470 mg in $5.0 \mathrm{~mL}$ THF, $5.0 \mathrm{mmol}$ ) was added via syringe. The mixture was allowed to warm to room temperature over 30 minutes and stirred for another 30 minutes. The resulting yellow solution was poured into $\mathrm{NH}_{4} \mathrm{Claq}$ and extracted with EtOAc three times. The combined organic layers were dried over $\mathrm{Na}_{2} \mathrm{SO}_{4}$, concentrated in vacuo, and the residue was subjected to silica gel chromatography (eluent: hexane/EtOAc $=10 / 1)$. The obtained white solid (contains $\sim 5 \%$ triptycene) was further purified by recrystallization (pentane vapor diffusion into THF solution) to give Trip-SMe as colorless blocks in 84\% yield (1.06 g, $3.51 \mathrm{mmol})$. m.p. $230-231{ }^{\circ} \mathrm{C} ;{ }^{1} \mathrm{H}$ NMR $\left(400 \mathrm{MHz}, \mathrm{CDCl}_{3}\right)$ 7.44-7.47 (m, 6H), 7.93-7.97 (m, 4H); ${ }^{13} \mathrm{C} \mathrm{NMR}(100 \mathrm{MHz}$, $\left.\mathrm{CDCl}_{3}\right)$ 109.0, 126.6, 129.2, 130.8, 133.1, 147.5, 167.9; HRMS (APCI) $m / z\left([\mathrm{M}+\mathrm{H}]^{+}\right)$calcd for $\mathrm{C}_{21} \mathrm{H}_{17} \mathrm{~S}$ : 301.1045, found: 301.1045 .

\section{3-2. Preparation of Trip-OMe}

A two-neck round-bottom flask equipped with an $\mathrm{N}_{2}$ balloon and a rubber cap was charged with 9methoxyanthracene (42 mg, $0.2 \mathrm{mmol}), \mathrm{CsF}(61 \mathrm{mg}, 0.4 \mathrm{mmol})$, and $\mathrm{MeCN}$ (2.0 mL). 2(Trimethylsilyl)phenyl trifluoromethanesulfonate $(90 \mathrm{mg}, 0.3 \mathrm{mmol})$ was added via syringe and the mixture was stirred at $80^{\circ} \mathrm{C}$ for $16 \mathrm{~h}$. The resulting suspension was filtered through a pad of Celite, and the filtrate was concentrated under reduced pressure. The residue was subjected to silica gel chromatography (eluent: hexane/EtOAc $=20 / 1)$ to give the title compound as white solid (56 mg, 99\% yield).

NMR data was identical to the reported value. ${ }^{7}$

\section{3-3. Multi-halogenation of naphthalene (Scheme 2)}

\section{Bromination (1b- $\left.\mathrm{Br}_{3}\right)$}

To an oven-dried Schlenk tube was added naphthalene $(\mathbf{1 b}, 64 \mathrm{mg}, 0.5 \mathrm{mmol})$, Trip-SMe $(7.5 \mathrm{mg}, 5.0$ mol\%), $\operatorname{AgSbF}_{6}(1.7 \mathrm{mg}, 1.0 \mathrm{~mol} \%)$, and DCE $(6.0 \mathrm{~mL})$. NBS $(312 \mathrm{mg}, 1.75 \mathrm{mmol})$ was added in one portion, and the mixture was stirred at room temperature for $24 \mathrm{~h}$. The resulting suspension was poured into $\mathrm{NaHCO}_{3}$ aq and extracted with $\mathrm{CHCl}_{3}$ three times. The combined organic layer was dried over $\mathrm{Na}_{2} \mathrm{SO}_{4}$ and 
concentrated in vacuo. The crude material $\left(\mathbf{1 b}-\mathrm{Br}_{3}: 78 \%\right.$ NMR yield) was subjected to silica gel chromatography (eluent: hexane) and $\mathrm{GPC}\left(\mathrm{CHCl}_{3}\right)$ to give the title compound as white solid $(128 \mathrm{mg}, 70 \%$ yield).

\section{1,4,6-Tribromonaphthalene $\left(1 \mathrm{~b}-\mathrm{Br}_{3}\right)^{8}$}<smiles>Brc1ccc2c(Br)ccc(Br)c2c1</smiles>

m.p. 84-85 ${ }^{\circ} \mathrm{C} ;{ }^{1} \mathrm{H}$ NMR (400 MHz, $\left.\mathrm{CDCl}_{3}\right) \delta 7.58-7.65$ (m, 2H), $7.68(\mathrm{dd}, J=9.0,1.9 \mathrm{~Hz}, 1 \mathrm{H}), 8.09$ (dd, $J=9.0,0.3 \mathrm{~Hz}, 1 \mathrm{H}), 8.40(\mathrm{dd}, J=1.9,0.3 \mathrm{~Hz}, 1 \mathrm{H}) ;{ }^{13} \mathrm{C} \mathrm{NMR}\left(100 \mathrm{MHz}, \mathrm{CDCl}_{3}\right) \delta 121.34,122.62,123.13$, 129.71, 130.07, 130.59, 131.23, 131.64, 131.73, 134.02; HRMS (EI) $m / z\left([\mathrm{M}]^{+}\right)$calcd for $\mathrm{C}_{10} \mathrm{H}_{5} \mathrm{Br}_{3}$ : 361.7941, found: 361.7937 .

\section{Chlorination $\left(\mathbf{1 b}-\mathrm{Cl}_{4}\right)$}

To an oven-dried Schlenk tube was added naphthalene $(\mathbf{1 b}, 64 \mathrm{mg}, 0.5 \mathrm{mmol})$, Trip-SMe $(7.5 \mathrm{mg}, 5.0$ mol\%), $\operatorname{AgSbF}_{6}(1.7 \mathrm{mg}, 1.0 \mathrm{~mol} \%)$, and DCE (6.0 mL). NCS (334 mg, $2.5 \mathrm{mmol}$ ) was added in one portion, and the mixture was stirred at room temperature for $72 \mathrm{~h}$. The resulting suspension was poured into $\mathrm{NaHCO}_{3}$ aq and extracted with $\mathrm{CHCl}_{3}$ three times. The combined organic layer was dried over $\mathrm{Na}_{2} \mathrm{SO}_{4}$ and concentrated in vacuo. The crude material (1b-Cl 4 : $78 \%$ NMR yield) was subjected to silica gel chromatography (eluent: hexane) and GPC $\left(\mathrm{CHCl}_{3}\right)$ to give the title compound as white solid $(97 \mathrm{mg}, 73 \%$ yield). Single crystals suitable for X-ray analysis were obtained from $\mathrm{CHCl}_{3}$ solution.

\section{1,4,5,8-Tetrachloronaphthalene $\left(1 \mathrm{~b}-\mathrm{Cl}_{4}\right)$}<smiles>Clc1ccc(Cl)c2c(Cl)ccc(Cl)c12</smiles>

m.p. $176-177{ }^{\circ} \mathrm{C} ;{ }^{1} \mathrm{H} \mathrm{NMR}\left(400 \mathrm{MHz}, \mathrm{CDCl}_{3}\right) \delta 7.54(\mathrm{~s}, 4 \mathrm{H}) ;{ }^{13} \mathrm{C} \mathrm{NMR}\left(100 \mathrm{MHz}, \mathrm{CDCl}_{3}\right) \delta 130.60,131.05$, 131.17; HRMS (EI) $m / z\left([M]^{+}\right)$calcd for $\mathrm{C}_{10} \mathrm{H}_{4} \mathrm{Cl}_{4}$ : 263.9067, found: 263.9063 . 


\section{3-4. Bromination of BINOL (Scheme 3)}

To an oven-dried Schlenk tube was added $(R)$-BINOL (1c, $143 \mathrm{mg}, 0.5 \mathrm{mmol})$, Trip-SMe $(7.5 \mathrm{mg}, 5.0$ mol\%), $\operatorname{AgSbF}_{6}(1.7 \mathrm{mg}, 1.0 \mathrm{~mol} \%)$, and DCE (4.0 mL). NBS (231 mg, $\left.1.3 \mathrm{mmol}\right)$ was added in one portion, and the mixture was stirred at room temperature for $4 \mathrm{~h}$. The resulting suspension was poured into $\mathrm{NaHCO}_{3} \mathrm{aq}$ and extracted with $\mathrm{CHCl}_{3}$ three times. The combined organic layer was dried over $\mathrm{Na}_{2} \mathrm{SO}_{4}$ and concentrated in vacuo. The crude material was subjected to silica gel chromatography (eluent: hexane/EtOAc $=5 / 1$ to $2 / 1)$ and GPC $\left(\mathrm{CHCl}_{3}\right)$ to give $1 \mathbf{c}-\mathrm{Br}_{2}$ as white solid (204 mg, 92\% yield). The enantiomeric purity was confirmed by HPLC analysis in comparison with authentic 6,6'-Dibromo-(S)BINOL sample (CHIRAL ART Cellulose-SB column, hexane $/$ EtOAc $=80 / 20,1.0 \mathrm{~mL} / \mathrm{min}, R$ isomer: $\mathrm{t}_{\mathrm{R}}=$ $18.2 \mathrm{~min}, S$ isomer: $\mathrm{t}_{\mathrm{R}}=18.9 \mathrm{~min}, \mathrm{UV}$ detection at $350 \mathrm{~nm}$ ).

6,6'-Dibromo-(R)-BINOL $\left(1 \mathrm{c}-\mathrm{Br}_{2}\right)^{9}$<smiles>Oc1ccc2cc(Br)ccc2c1-c1c(O)ccc2cc(Br)ccc12</smiles>

${ }^{1} \mathrm{H}$ NMR $\left(400 \mathrm{MHz}, \mathrm{CDCl}_{3}\right) \delta 5.03(\mathrm{~s}, 2 \mathrm{H}), 6.96(\mathrm{~d}, J=8.9 \mathrm{~Hz}, 2 \mathrm{H}), 7.33-7.46(\mathrm{~m}, 4 \mathrm{H}), 7.90(\mathrm{~d}, J=8.9$ $\mathrm{Hz}, 2 \mathrm{H}), 8.06(\mathrm{~d}, J=1.9 \mathrm{~Hz}, 2 \mathrm{H}) ;{ }^{13} \mathrm{C} \mathrm{NMR}\left(100 \mathrm{MHz}, \mathrm{CDCl}_{3}\right) \delta 110.75,118.17,119.13,126.02,130.61$, $130.74,130.87,131.02,132.02,153.13$.

[HPLC chart for the product]

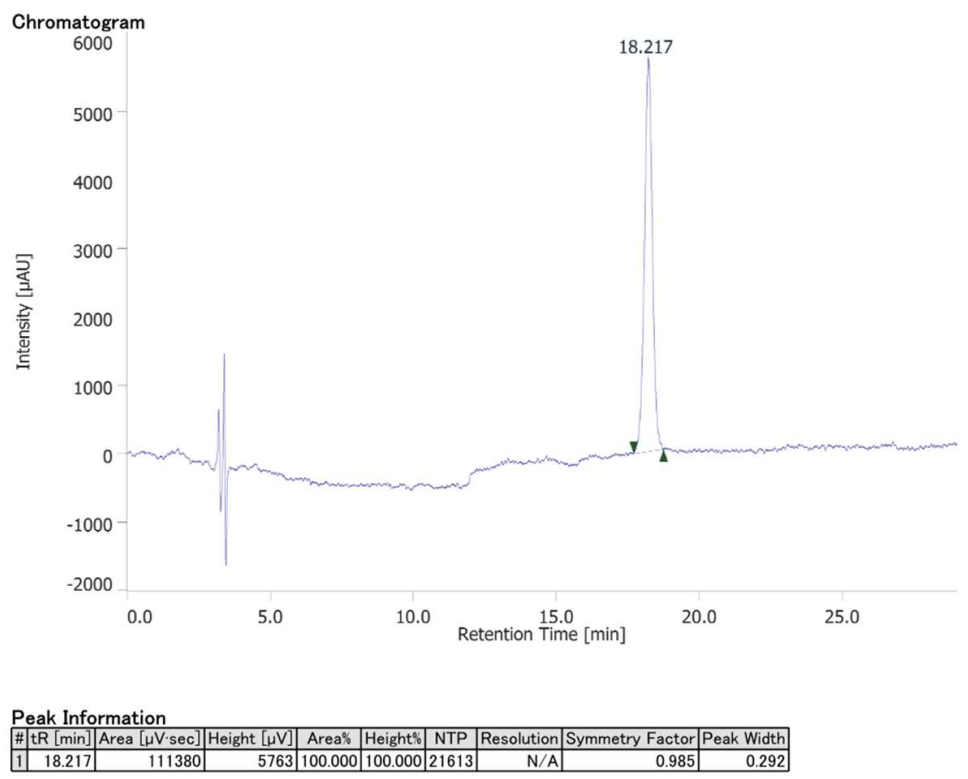


[HPLC chart for the authentic $S$ isomer]

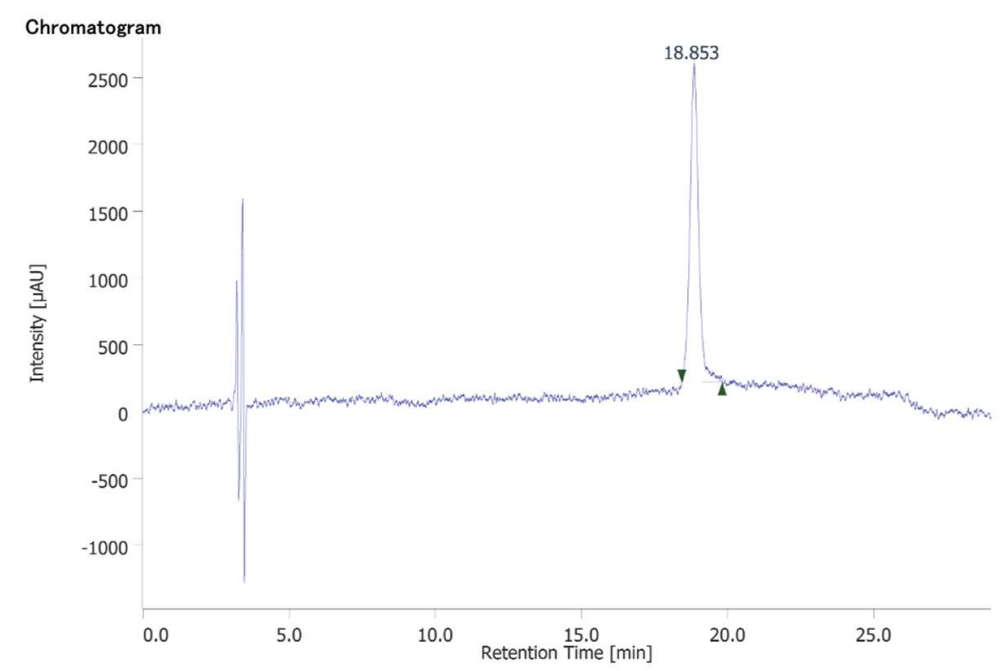

Peak Information

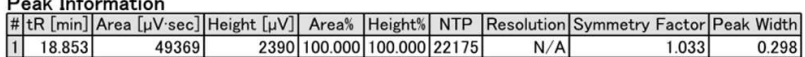




\section{3-5. General procedure for the Trip-SMe-catalyzed halogenation (Scheme 4 and 5 )}

To an oven-dried Schlenk tube was added the aromatic compound $1(0.5 \mathrm{mmol})$, Trip-SMe $(7.5 \mathrm{mg}, 5.0$ mol\%), $\operatorname{AgSbF}_{6}(1.7 \mathrm{mg}, 1.0 \mathrm{~mol} \%$ ), and DCE (3 mL otherwise noted). NXS was added in one portion, and the mixture was stirred at room temperature for the specified period. The resulting suspension was poured into $\mathrm{NaHCO}_{3}$ aq and extracted with $\mathrm{CHCl}_{3}$ three times. The combined organic layer was dried over $\mathrm{Na}_{2} \mathrm{SO}_{4}$ and concentrated in vacuo. The crude material was subjected to silica gel chromatography.

\section{4,4'-dibromo-1,1'-biphenyl $\left(1 \mathrm{~d}-\mathrm{Br}_{2}\right)^{10}$}

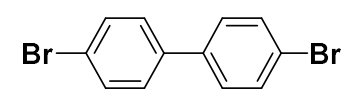

Isolated by silica gel chromatography (hexane/EtOAc $=40 / 1)\left(152 \mathrm{mg}, 98 \%\right.$ yield), white solid; ${ }^{1} \mathrm{H}$ NMR $\left(400 \mathrm{MHz}, \mathrm{CDCl}_{3}\right) \delta$ 7.38-7.43 (m, 4H), 7.53-7.58 (m, 4H); ${ }^{13} \mathrm{C} \mathrm{NMR}\left(100 \mathrm{MHz}, \mathrm{CDCl}_{3}\right) \delta 122.09,128.65$, $132.16,139.04$.

\section{4,4"-dibromo-p-terphenyl $\left(1 \mathrm{e}-\mathrm{Br}_{2}\right)^{11}$}

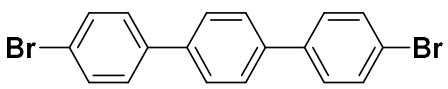

Analytically pure compound was obtained after washing the crude material with $\mathrm{CHCl}_{3}(166 \mathrm{mg}, 86 \%)$, white solid; ${ }^{1} \mathrm{H}$ NMR $\left(400 \mathrm{MHz}, \mathrm{CDCl}_{3} / \mathrm{CS}_{2}=1 / 1\right) \delta$ 7.44-7.48 (m, 4H), 7.52-7.56 (m, 4H), 7.59 (s, 4H); ${ }^{13} \mathrm{C} \mathrm{NMR}\left(100 \mathrm{MHz}, \mathrm{CDCl}_{3} / \mathrm{CS}_{2}=1 / 1\right) \delta 122.03,127.45,128.57,132.02,139.25,139.37$.

\section{2,8- Dibromodibenzo $[\boldsymbol{b}, \boldsymbol{d}]$ furan $\left(1 \mathrm{f}-\mathrm{Br}_{2}\right)^{12}$}<smiles>Brc1ccc2oc3ccc(Br)cc3c2c1</smiles>

Isolated by silica gel chromatography (hexane) and $\mathrm{GPC}\left(\mathrm{CHCl}_{3}\right)\left(110 \mathrm{mg}, 67 \%\right.$ yield), white solid; ${ }^{1} \mathrm{H}$ $\operatorname{NMR}\left(400 \mathrm{MHz}, \mathrm{CDCl}_{3}\right) \delta 7.45(\mathrm{~d}, J=8.7 \mathrm{~Hz}, 2 \mathrm{H}), 7.58(\mathrm{dd}, J=8.7,2.0 \mathrm{~Hz}, 2 \mathrm{H}), 8.03(\mathrm{~d}, J=1.9 \mathrm{~Hz}$, $2 \mathrm{H}) ;{ }^{13} \mathrm{C}$ NMR $\left(100 \mathrm{MHz}, \mathrm{CDCl}_{3}\right) \delta 113.55,116.08,123.85,125.30,130.89,155.48$. 
2,8-Dibromodibenzo $[\boldsymbol{b}, \boldsymbol{d}]$ thiophene $\left(1 \mathrm{~g}-\mathrm{Br}_{2}\right)^{13}$<smiles>Brc1ccc2sc3ccc(Br)cc3c2c1</smiles>

Isolated by silica gel chromatography (hexane) and $\mathrm{GPC}\left(\mathrm{CHCl}_{3}\right)(138 \mathrm{mg}, 81 \%)$, white solid; ${ }^{1} \mathrm{H} \mathrm{NMR}$ $\left(400 \mathrm{MHz}, \mathrm{CDCl}_{3}\right) \delta 7.58(\mathrm{dd}, J=8.5,1.9 \mathrm{~Hz}, 2 \mathrm{H}), 7.71(\mathrm{~d}, J=8.5 \mathrm{~Hz}, 2 \mathrm{H}), 8.23(\mathrm{~d}, J=1.9 \mathrm{~Hz}, 2 \mathrm{H}) ;{ }^{13} \mathrm{C}$ NMR $\left(100 \mathrm{MHz}, \mathrm{CDCl}_{3}\right) \delta 118.76,124.32,124.85,130.43,136.29,138.75$.

\section{2,2',7,7'-tetrabromo-9,9'-spirobi[fluorene] $\left(1 \mathrm{~h}-\mathrm{Br}_{4}\right)^{14}$}<smiles></smiles>

$12.0 \mathrm{~mL}$ of DCE solvent was used. Isolated by silica gel chromatography (hexane/EtOAc $=40 / 1)$ and $\mathrm{GPC}\left(\mathrm{CHCl}_{3}\right)(306 \mathrm{mg}, 97 \%)$, single crystals suitable for X-ray analysis were obtained by slow evaporation of $\mathrm{CHCl}_{3}$ solution, white solid; ${ }^{1} \mathrm{H} \mathrm{NMR}\left(400 \mathrm{MHz}, \mathrm{CDCl}_{3}\right) \delta 6.83(\mathrm{~d}, J=1.5 \mathrm{~Hz}, 4 \mathrm{H}), 7.54$ (dd, $J=8.2$, $1.8 \mathrm{~Hz}, 4 \mathrm{H}), 7.68(\mathrm{~d}, J=8.2 \mathrm{~Hz}, 4 \mathrm{H}) ;{ }^{13} \mathrm{C} \mathrm{NMR}\left(100 \mathrm{MHz}, \mathrm{CDCl}_{3}\right) \delta 65.27,121.84,122.32,127.48,131.91$, 139.70, 148.93 .

4,7,12,15-Tetrabromo[2,2]paracyclophane $\left(1 \mathrm{i}-\text { para }-\mathrm{Br}_{4}\right)^{15}$ and 4,5,12,13-Tetrabromo[2,2]paracyclophane $\left(1 \mathrm{i} \text {-ortho- } \mathrm{Br}_{4}\right)^{15}$

$6.0 \mathrm{~mL}$ of DCE solvent was used. The products were isolated by silica gel chromatography (hexane) and GPC (EtOAc)

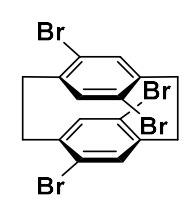

(85 mg, 33\% yield), white solid; ${ }^{1} \mathrm{H}$ NMR (400 MHz, $\left.\mathrm{CDCl}_{3}\right) \delta 2.92-3.06(\mathrm{~m}, 4 \mathrm{H}), 3.17-3.30(\mathrm{~m}, 4 \mathrm{H}), 7.20$ (s, 4H); ${ }^{13} \mathrm{C} \mathrm{NMR}\left(100 \mathrm{MHz}, \mathrm{CDCl}_{3}\right) \delta 32.84,125.44,134.57,140.47$.

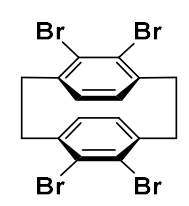


(80 mg, 30\% yield), white solid; ${ }^{1} \mathrm{H}$ NMR (400 MHz, $\left.\mathrm{CDCl}_{3}\right) \delta$ 3.04-3.16 (m, 4H), 3.29-3.42 (m, 4H), 6.99 $(\mathrm{s}, 4 \mathrm{H}) ;{ }^{13} \mathrm{C} \mathrm{NMR}\left(100 \mathrm{MHz}, \mathrm{CDCl}_{3}\right) \delta 34.70,128.79,129.43,140.84$.

\section{5-Chloro-1,2,3,4-tetrahydronaphthalene $(\mathbf{1 j}-\mathrm{Cl}(\mathrm{C} 1))^{16}$}<smiles>Clc1cccc2c1CCCC2</smiles>

Isolated by silica gel chromatography (hexane) and GPC (EtOAc) $\left(30 \mathrm{mg}, 36 \%\right.$ yield), colorless oil; ${ }^{1} \mathrm{H}$ NMR (400 MHz, $\left.\mathrm{CDCl}_{3}\right) \delta 1.71-1.88(\mathrm{~m}, 4 \mathrm{H}), 2.69-2.83(\mathrm{~m}, 4 \mathrm{H}), 6.94-7.06(\mathrm{~m}, 2 \mathrm{H}), 7.14-7.20(\mathrm{~m}, 1 \mathrm{H})$; ${ }^{13} \mathrm{C}$ NMR $\left(100 \mathrm{MHz}, \mathrm{CDCl}_{3}\right) \delta 22.76,23.13,27.29,30.07,126.25,126.45,127.68,134.76,135.06,139.68$.

\section{6-Chloro-1,2,3,4-tetrahydrolnaphthalene $(\mathbf{1 j}-\mathrm{Cl}(\mathrm{C} 2))^{17}$}<smiles>Clc1ccc2c(c1)CCCC2</smiles>

Isolated by silica gel chromatography (hexane) and GPC (EtOAc) (19 mg, 23\% yield), colorless oil; ${ }^{1} \mathrm{H}$ NMR (400 MHz, $\left.\mathrm{CDCl}_{3}\right) \delta$ 1.72-1.84 (m, 4H), 2.65-2.80 (m, 4H), 6.94-7.00 (m, 1H), 7.01-7.08 (m, 2H); ${ }^{13} \mathrm{C}$ NMR $\left(100 \mathrm{MHz}, \mathrm{CDCl}_{3}\right) \delta 22.96,23.13,28.95,29.39,125.63,128.92,130.49,130.89,135.63,139.07$.

\section{A mixture of 5-bromo-1,2,3,4-tetrahydronaphthalene ${ }^{18}$}

and 6-bromo-1,2,3,4-tetrahydronaphthalene $(\mathbf{1} \mathbf{j}-\mathrm{Br})^{19}$<smiles>Brc1ccc2c(c1)CCCCC2</smiles>

Isolated by silica gel chromatography (hexane) (101 mg, 96\% yield), colorless oil; ${ }^{1} \mathrm{H}$ NMR (400 MHz, $\left.\mathrm{CDCl}_{3}\right) \delta 1.68-1.87(\mathrm{~m}, 4 \mathrm{H}), 2.63-2.81(\mathrm{~m}, 4 \mathrm{H}), 6.87-6.98(\mathrm{~m}, 0.22 \times 1 \mathrm{H}+0.78 \times 1 \mathrm{H}), 7.01(\mathrm{~d}, J=7.6 \mathrm{~Hz}$, $0.22 \times 1 \mathrm{H}), 7.15-7.23(\mathrm{~m}, 0.78 \times 2 \mathrm{H}), 7.36(\mathrm{~d}, J=7.6 \mathrm{~Hz}, 0.22 \times 1 \mathrm{H}) ;{ }^{13} \mathrm{C} \mathrm{NMR}\left(100 \mathrm{MHz}, \mathrm{CDCl}_{3}\right) \delta 22.82$, $22.93,23.05,23.48,29.01,29.33,30.28,30.32,118.96,126.02,126.69,128.40,128.52,129.82,130.85$, $131.87,136.16,136.59,139.53,139.96$. 


\section{A mixture of 5-iodo-1,2,3,4-tetrahydronaphthalene}

and 6-iodo-1,2,3,4-tetrahydronaphthalene $(1 \mathrm{j}-\mathrm{I})$<smiles>CC[C@@H]1CCCc2cc(I)ccc21</smiles>

Isolated by silica gel chromatography (hexane) $\left(118 \mathrm{mg}, 92 \%\right.$ yield), colorless oil; ${ }^{1} \mathrm{H}$ NMR (400 MHz, $\left.\mathrm{CDCl}_{3}\right) \delta 1.68-1.87(\mathrm{~m}, 4 \mathrm{H}), 2.60-2.80(\mathrm{~m}, 4 \mathrm{H}), 6.75-6.84(\mathrm{~m}, 0.30 \times 1 \mathrm{H}+0.70 \times 1 \mathrm{H}), 7.04(\mathrm{~d}, J=7.6 \mathrm{~Hz}$, $0.30 \times 1 \mathrm{H}), 7.35-7.43(\mathrm{~m}, 0.70 \times 2 \mathrm{H}), 7.66(\mathrm{~d}, J=7.7 \mathrm{~Hz}, 0.30 \times 1 \mathrm{H}) ;{ }^{13} \mathrm{C} \mathrm{NMR}\left(100 \mathrm{MHz}, \mathrm{CDCl}_{3}\right) \delta 22.91$, 22.98, 23.01, 24.20, 29.08, 29.15, 30.58, 36.05, 90.43, 103.56, 127.21, 129.45, 131.14, 134.46, 136.66, 136.92, 137.94, 139.50, 139.65, 139.91; HRMS (EI) $\mathrm{m} / z\left([\mathrm{M}]^{+}\right)$calcd for $\mathrm{C}_{10} \mathrm{H}_{11} \mathrm{I}: 257.9906$, found: 257.9904.

\section{2-Chloro-1,3,5-trimethylbenzene $(1 \mathrm{~m}-\mathrm{Cl})^{20}$}<smiles>Cc1cc(C)c(Cl)c(C)c1</smiles>

Isolated by silica gel chromatography (hexane) $\left(47 \mathrm{mg}, 60 \%\right.$ yield), colorless oil; ${ }^{1} \mathrm{H}$ NMR $(400 \mathrm{MHz}$, $\left.\mathrm{CDCl}_{3}\right) \delta 2.24(\mathrm{~s}, 3 \mathrm{H}), 2.33(\mathrm{~s}, 6 \mathrm{H}), 6.88(\mathrm{~d}, J=0.6 \mathrm{~Hz}, 2 \mathrm{H}) ;{ }^{13} \mathrm{C} \mathrm{NMR}\left(100 \mathrm{MHz}, \mathrm{CDCl}_{3}\right): \delta 20.72,20.81$, $129.29,131.67,135.67,135.96$.

\section{2-Bromo-1,3,5-trimethylbenzene $(1 \mathrm{~m}-\mathrm{Br})^{20}$}<smiles>Cc1cc(C)c(Br)c(C)c1</smiles>

Isolated by silica gel chromatography (hexane/EtOAc $=20 / 1)(97 \mathrm{mg}, 97 \%$ yield $)$, colorless oil; ${ }^{1} \mathrm{H}$ NMR $\left(400 \mathrm{MHz}, \mathrm{CDCl}_{3}\right) \delta 2.23(\mathrm{~s}, 3 \mathrm{H}), 2.36(\mathrm{~s}, 6 \mathrm{H}), 6.88(\mathrm{~d}, J=0.4 \mathrm{~Hz}, 2 \mathrm{H}) ;{ }^{13} \mathrm{C} \mathrm{NMR}\left(100 \mathrm{MHz}, \mathrm{CDCl}_{3}\right) \delta$ $20.80,23.85,124.32,129.15,136.40,138.02$. 
9,10-Dichloroanthracene $\left(1 \mathrm{n}-\mathrm{Cl}_{2}\right)^{21}$<smiles>Clc1c2ccccc2c(Cl)c2ccccc12</smiles>

$6.0 \mathrm{~mL}$ of DCE solvent was used. Isolated by silica gel chromatography (hexane) (117 mg, 95\% yield), yellow solid; ${ }^{1} \mathrm{H}$ NMR $\left(400 \mathrm{MHz}, \mathrm{CDCl}_{3}\right) \delta$ 7.62-7.69 (m, 4H), 8.53-8.60 (m, 4H); ${ }^{13} \mathrm{C} \mathrm{NMR}(100 \mathrm{MHz}$, $\left.\mathrm{CDCl}_{3}\right) \delta 125.37,127.26,128.41,129.32$.

9,10-Dibromoanthracene $\left(1 \mathrm{n}-\mathrm{Br}_{2}\right)^{22}$<smiles>Brc1c2ccccc2c(Br)c2ccccc12</smiles>

$6.0 \mathrm{~mL}$ of DCE solvent was used. Isolated by silica gel chromatography (hexane) (154 mg, 92\% yield), yellow solid; ${ }^{1} \mathrm{H}$ NMR $\left(400 \mathrm{MHz}, \mathrm{CDCl}_{3}\right) \delta$ 7.61-7.68 (m, 4H), 8.56-8.64 (m, 4H); ${ }^{13} \mathrm{C} \mathrm{NMR}(100 \mathrm{MHz}$, $\left.\mathrm{CDCl}_{3}\right) \delta 123.69,127.62,128.44,131.20$.

Ethyl 3-chloro-1 $H$-indole-2-carboxylate $(10-\mathrm{Cl})^{23}$<smiles>CCOC(=O)c1[nH]c2ccccc2c1Cl</smiles>

Isolated by silica gel chromatography (hexane/EtOAc $=5 / 1)(109 \mathrm{mg}, 98 \%)$, white solid; ${ }^{1} \mathrm{H}$ NMR (400 $\left.\mathrm{MHz}, \mathrm{CDCl}_{3}\right) \delta 1.46(\mathrm{t}, J=7.1 \mathrm{~Hz}, 3 \mathrm{H}), 4.47(\mathrm{q}, J=7.1 \mathrm{~Hz}, 3 \mathrm{H}), 7.19-7.25(\mathrm{~m}, 1 \mathrm{H}), 7.34-7.42(\mathrm{~m}, 2 \mathrm{H})$, 7.69-7.75 (m, 1H), $9.01(\mathrm{~s}, 1 \mathrm{H}) ;{ }^{13} \mathrm{C}$ NMR (100 MHz, $\left.\mathrm{CDCl}_{3}\right) \delta 14.49,61.59,112.21,112.57,120.35$, $121.40,122.52,126.34,126.68,134.90,161.25$.

\section{Butyl 5-bromofuran-2-carboxylate (1p-Br)}<smiles>CCCCOC(=O)c1ccc(Br)o1</smiles>

Isolated by silica gel chromatography (hexane/EtOAc $=40 / 1)$ and GPC (EtOAc) $(83 \mathrm{mg}, 67 \%)$, colorless oil; ${ }^{1} \mathrm{H}$ NMR (400 MHz, $\left.\mathrm{CDCl}_{3}\right) \delta 0.97(\mathrm{t}, J=7.4 \mathrm{~Hz}, 3 \mathrm{H}), 1.38-1.50(\mathrm{~m}, 2 \mathrm{H}), 1.68-1.77(\mathrm{~m}, 2 \mathrm{H}), 4.30(\mathrm{t}$, $J=6.7 \mathrm{~Hz}, 2 \mathrm{H}), 6.45(\mathrm{~d}, J=3.5 \mathrm{~Hz}, 1 \mathrm{H}), 7.11(\mathrm{~d}, J=3.5 \mathrm{~Hz}, 1 \mathrm{H}) ;{ }^{13} \mathrm{C} \mathrm{NMR}\left(100 \mathrm{MHz}, \mathrm{CDCl}_{3}\right) \delta 13.83$, 
$19.23,30.80,65.20,113.98,119.96,127.48,146.63,157.91$; HRMS (APCI) $m / z\left([\mathrm{M}+\mathrm{H}]^{+}\right)$calcd for $\mathrm{C}_{9} \mathrm{H}_{12} \mathrm{BrO}_{3}$ : 246.9964, found: 246.9942.

Butyl 5-chlorothiophene-2-carboxylate (1q-Cl)<smiles>CCCCOC(=O)c1ccc(Cl)s1</smiles>

Isolated by silica gel chromatography (hexane/EtOAc $=40 / 1)$ and GPC $(\mathrm{EtOAc})(70 \mathrm{mg}, 64 \%)$, pale yellow oil; ${ }^{1} \mathrm{H}$ NMR (400 MHz, $\left.\mathrm{CDCl}_{3}\right) \delta 0.97$ (t, $\left.J=7.4 \mathrm{~Hz}, 3 \mathrm{H}\right), 1.38-1.50(\mathrm{~m}, 2 \mathrm{H}), 1.66-1.77$ (m, 2H), 4.28 (t, $J=6.6 \mathrm{~Hz}, 2 \mathrm{H}), 6.93(\mathrm{~d}, J=4.0 \mathrm{~Hz}, 1 \mathrm{H}), 7.58(\mathrm{~d}, J=4.0 \mathrm{~Hz}, 1 \mathrm{H}) ;{ }^{13} \mathrm{C} \mathrm{NMR}\left(100 \mathrm{MHz}, \mathrm{CDCl}_{3}\right) \delta 13.83$, 19.28, 30.78, 65.36, 127.31, 132.30, 132.89, 137.23, 161.43; HRMS (APCI) $\mathrm{m} / z\left([\mathrm{M}+\mathrm{H}]^{+}\right)$calcd for $\mathrm{C}_{9} \mathrm{H}_{12} \mathrm{ClO}_{2} \mathrm{~S}: 219.0241$, found: 219.0212 .

\section{4-Bromophenol (1r-Br) $)^{24}$}<smiles>Oc1ccc(Br)cc1</smiles>

Isolated by silica gel chromatography (hexane/EtOAc $=5 / 1)\left(73 \mathrm{mg}, 84 \%\right.$ yield), brown oil; ${ }^{1} \mathrm{H}$ NMR (400 $\left.\mathrm{MHz}, \mathrm{CDCl}_{3}\right) \delta 5.51(\mathrm{~s}, 1 \mathrm{H}), 6.67-6.77(\mathrm{~m}, 2 \mathrm{H}), 7.28-7.37(\mathrm{~m}, 2 \mathrm{H}) ;{ }^{13} \mathrm{C} \mathrm{NMR}\left(100 \mathrm{MHz}, \mathrm{CDCl}_{3}\right) \delta 113.00$, $117.34,132.61,154.74$.

\section{4-Bromoaniline $(1 \mathrm{~s}-\mathrm{Br})^{25}$}<smiles>Nc1ccc(Br)cc1</smiles>

Isolated by silica gel chromatography (hexane/EtOAc $=10 / 1$ to $5 / 1)\left(66 \mathrm{mg}, 76 \%\right.$ yield), brown solid; ${ }^{1} \mathrm{H}$ $\operatorname{NMR}\left(400 \mathrm{MHz}, \mathrm{CDCl}_{3}\right) \delta 3.66(\mathrm{~s}, 2 \mathrm{H}), 6.52-6.61(\mathrm{~m}, 2 \mathrm{H}), 7.20-7.25(\mathrm{~m}, 2 \mathrm{H}) ;{ }^{13} \mathrm{C} \mathrm{NMR}\left(100 \mathrm{MHz}, \mathrm{CDCl}_{3}\right)$ $\delta 110.34,116.83,132.15,145.54$.

\section{Methyl 5-chloro-2-methoxybenzoate $(1 \mathrm{t}-\mathrm{Cl})^{26}$}<smiles>COC(=O)c1cc(Cl)ccc1OC</smiles>

Isolated by silica gel chromatography (hexane/EtOAc $=10 / 1)(91 \mathrm{mg}, 91 \%$ yield $)$, colorless oil; ${ }^{1} \mathrm{H}$ NMR $\left(400 \mathrm{MHz}, \mathrm{CDCl}_{3}\right) \delta 3.89$ (s, 3H), $3.90(\mathrm{~s}, 3 \mathrm{H}), 6.92(\mathrm{~d}, J=8.9 \mathrm{~Hz}, 1 \mathrm{H}), 7.42(\mathrm{dd}, J=8.9,2.7 \mathrm{~Hz}, 1 \mathrm{H})$, 
$7.77(\mathrm{~d}, J=2.7 \mathrm{~Hz}, 1 \mathrm{H}) ;{ }^{13} \mathrm{C} \mathrm{NMR}\left(100 \mathrm{MHz}, \mathrm{CDCl}_{3}\right) \delta 52.40,56.48,113.57,121.35,125.33,131.51$, $133.28,157.90,165.55$.

\section{Methyl 5-bromo-2-methoxybenzoate (1t-Br) $)^{27}$}<smiles>COC(=O)c1cc(Br)ccc1OC</smiles>

Isolated by silica gel chromatography (hexane/EtOAc $=9 / 1)\left(121 \mathrm{mg}, 99 \%\right.$ yield), pale yellow oil; ${ }^{1} \mathrm{H}$ NMR $\left(400 \mathrm{MHz}, \mathrm{CDCl}_{3}\right) \delta 3.89$ (s, 3H), 3.89 (s, 3H), 6.87 (d, $\left.J=8.9 \mathrm{~Hz}, 1 \mathrm{H}\right), 7.55$ (dd, $\left.J=8.9,2.6 \mathrm{~Hz}, 1 \mathrm{H}\right)$, $7.91(\mathrm{~d}, J=2.6 \mathrm{~Hz}, 1 \mathrm{H}) ;{ }^{13} \mathrm{C} \mathrm{NMR}\left(100 \mathrm{MHz}, \mathrm{CDCl}_{3}\right) \delta 52.37,56.36,112.29,113.95,121.72,134.31$, $136.18,158.34,165.37$.

\section{1-Bromo-3,5-dichloro-2-methoxybenzene (1u-Br)}<smiles>COc1c(Cl)cc(Cl)cc1Br</smiles>

Isolated by silica gel chromatography (hexane/EtOAc $=20 / 1)(127 \mathrm{mg}, 99 \%$ yield), pale yellow solid, m.p. 59-60 ${ }^{\circ} \mathrm{C} ;{ }^{1} \mathrm{H}$ NMR $\left(400 \mathrm{MHz}, \mathrm{CDCl}_{3}\right) \delta 3.87(\mathrm{~s}, 3 \mathrm{H}), 7.34(\mathrm{~d}, J=2.4 \mathrm{~Hz}, 1 \mathrm{H}), 7.46(\mathrm{~d}, J=2.4 \mathrm{~Hz}, 1 \mathrm{H})$; ${ }^{13} \mathrm{C}$ NMR $\left(100 \mathrm{MHz}, \mathrm{CDCl}_{3}\right) \delta 60.91,118.94,129.62,129.86,130.14,131.69,152.49$; HRMS (EI) $\mathrm{m} / \mathrm{z}$ $\left([\mathrm{M}+\mathrm{H}]^{+}\right)$calcd for $\mathrm{C}_{7} \mathrm{H}_{5} \mathrm{BrCl}_{2} \mathrm{O}$ : 253.8901, found: 253.8902 .

\section{$\mathrm{N}$-(4-bromo-2-(trifluoromethyl)phenyl)acetamide $(1 \mathrm{v}-\mathrm{Br})^{28}$<smiles>CNc1ccc(Br)cc1C(F)(F)F</smiles>

Isolated by silica gel chromatography (hexane/EtOAc $=5 / 1$ to $2 / 1)\left(136 \mathrm{mg}, 96 \%\right.$ yield), white solid; ${ }^{1} \mathrm{H}$ $\operatorname{NMR}\left(400 \mathrm{MHz}, \mathrm{CDCl}_{3}\right) \delta 2.22(\mathrm{~s}, 3 \mathrm{H}), 7.36(\mathrm{~s}, 1 \mathrm{H}), 7.66(\mathrm{dd}, J=8.8,1.8 \mathrm{~Hz}, 1 \mathrm{H}), 7.73(\mathrm{~d}, J=1.8 \mathrm{~Hz}$, $1 \mathrm{H}), 8.13(\mathrm{~d}, J=8.5 \mathrm{~Hz}, 1 \mathrm{H}) ;{ }^{13} \mathrm{C} \mathrm{NMR}\left(100 \mathrm{MHz}, \mathrm{CDCl}_{3}\right) \delta 24.59,117.45,122.03$ (q, $\left.J=30.0 \mathrm{~Hz}\right), 123.11$ $(\mathrm{q}, J=273.4 \mathrm{~Hz}), 126.51,129.12(\mathrm{q}, J=5.3 \mathrm{~Hz}), 134.41,135.86,168.59 ;{ }^{19} \mathrm{~F}$ NMR $\left(376 \mathrm{MHz}, \mathrm{CDCl}_{3}\right) \delta-$ 60.97. 


\section{Methyl 5-bromo-2-pivalamidobenzoate (1w-Br)}<smiles>COC(=O)c1cc(Br)ccc1NP</smiles>

Isolated by silica gel chromatography (hexane/EtOAc $=20 / 1)$ and $\mathrm{GPC}\left(\mathrm{CHCl}_{3}\right)(139 \mathrm{mg}, 89 \%$ yield), white solid, m.p. $77-78{ }^{\circ} \mathrm{C} ;{ }^{1} \mathrm{H}$ NMR (400 MHz, $\left.\mathrm{CDCl}_{3}\right) \delta 1.34$ (s, 9H), 3.95 (s, 3H), 7.62 (dd, $J=9.1,2.5 \mathrm{~Hz}$, $1 \mathrm{H}), 8.16(\mathrm{~d}, J=2.5 \mathrm{~Hz}, 1 \mathrm{H}), 8.72(\mathrm{~d}, J=9.1 \mathrm{~Hz}, 1 \mathrm{H}), 11.25(\mathrm{~s}, 1 \mathrm{H}) ;{ }^{13} \mathrm{C} \mathrm{NMR}\left(100 \mathrm{MHz}, \mathrm{CDCl}_{3}\right) \delta 27.66$, $40.58,52.77,114.63,116.63,122.17,133.47,137.46,141.20,167.83,178.10 ;$ HRMS (APCI) m/z $\left([\mathrm{M}+\mathrm{H}]^{+}\right)$calcd for $\mathrm{C}_{13} \mathrm{H}_{17} \mathrm{BrNO}_{3}: 314.0386$, found: 314.0385 .

\section{2-((4-Bromophenoxy)methyl)oxirane $(\mathbf{1 x}-\mathrm{Br})^{29}$}<smiles>Brc1ccc(OCC2CO2)cc1</smiles>

Isolated by silica gel chromatography (hexane/EtOAc $=5 / 1$ to 2/1) and $\mathrm{GPC}\left(\mathrm{CHCl}_{3}\right)(95 \mathrm{mg}, 83 \%$ yield), pale yellow oil; ${ }^{1} \mathrm{H}$ NMR (400 MHz, $\left.\mathrm{CDCl}_{3}\right) \delta 2.75(\mathrm{dd}, J=4.9,2.6 \mathrm{~Hz}, 1 \mathrm{H}), 2.91(\mathrm{dd}, J=4.9,4.2 \mathrm{~Hz}, 1 \mathrm{H})$, 3.30-3.38 (m, 1H), $3.90(\mathrm{dd}, J=11.0,5.8 \mathrm{~Hz}, 1 \mathrm{H}), 4.21(\mathrm{dd}, J=11.0,3.0 \mathrm{~Hz}, 1 \mathrm{H}), 6.77-6.84(\mathrm{~m}, 2 \mathrm{H}), 7.34-$ $7.42(\mathrm{~m}, 2 \mathrm{H}) ;{ }^{13} \mathrm{C} \mathrm{NMR}\left(100 \mathrm{MHz}, \mathrm{CDCl}_{3}\right) \delta 44.74,50.14,69.12,113.59,116.59,132.46,157.72$.

\section{3-6. Bromination of strychnine (strychnidin-10-one) (Scheme 5)}

To an oven-dried Schlenk tube was added strychnidin-10-one $1 \mathbf{y}(0.5 \mathrm{mmol})$, Trip-SMe (7.5 mg, $5.0 \mathrm{~mol} \%)$, and DCE $(2.0 \mathrm{~mL})$. To the suspension was added TfOH (90 mg, 1.2 equiv in $3.0 \mathrm{~mL}$ DCE) dropwise. NBS ( 1.0 or 2.0 equiv) was added in one portion, and the mixture was stirred at room temperature for $24 \mathrm{~h}$. The resulting suspension was poured into $\mathrm{NaHCO}_{3}$ aq and extracted with $\mathrm{CHCl}_{3}$. The combined organic layer was dried over $\mathrm{Na}_{2} \mathrm{SO}_{4}$ and concentrated in vacuo. The crude material was subjected to silica gel chromatography (eluent: $\mathrm{CHCl}_{3} / \mathrm{MeOH}=20 / 1$ ).

\section{2-Bromostrychnidin-10-one (1y-Br)}<smiles>O=C1CC2C[C@@]34CCN5CC(=CCO2)C5C3N1c1ccc(Br)cc14</smiles> 
(199 mg, 96\% yield), white solid, m.p. $200{ }^{\circ} \mathrm{C}$ (decompose); ${ }^{1} \mathrm{H}$ NMR (400 MHz, $\left.\mathrm{CDCl}_{3}\right) \delta 1.25$ (dt, $J=$ 10.5, $3.2 \mathrm{~Hz}, 1 \mathrm{H}), 1.45$ (d, $J=14.4 \mathrm{~Hz}, 1 \mathrm{H}), 1.85-1.91$ (m, 2H), 2.36 (dt, $J=14.4,4.4 \mathrm{~Hz}, 1 \mathrm{H}), 2.65$ (dd, $J$ $=17.4,3.2 \mathrm{~Hz}, 1 \mathrm{H}), 2.71(\mathrm{~d}, J=14.9 \mathrm{~Hz}, 1 \mathrm{H}), 2.83-2.88(\mathrm{~m}, 1 \mathrm{H}), 3.09-3.22(\mathrm{~m}, 3 \mathrm{H}), 3.69(\mathrm{dd}, J=14.8$, $1.4 \mathrm{~Hz}, 1 \mathrm{H}), 3.85-3.88(\mathrm{~m}, 2 \mathrm{H}), 4.02-4.08(\mathrm{~m}, 1 \mathrm{H}), 4.12-4.17(\mathrm{~m}, 1 \mathrm{H}), 4.28(\mathrm{dt}, J=8.4,3.3 \mathrm{~Hz}, 1 \mathrm{H}), 5.90$ $(\mathrm{t}, J=4.4 \mathrm{~Hz}, 1 \mathrm{H}), 7.24(\mathrm{~d}, J=2.0 \mathrm{~Hz}, 1 \mathrm{H}), 7.35(\mathrm{dd}, J=8.6,2.0 \mathrm{~Hz}, 1 \mathrm{H}), 7.98(\mathrm{~d}, J=8.6 \mathrm{~Hz}, 1 \mathrm{H}) ;{ }^{13} \mathrm{C}$ $\operatorname{NMR}\left(100 \mathrm{MHz}, \mathrm{CDCl}_{3}\right) \delta 26.82,31.47,42.40,42.75,48.10,50.30,51.86,52.60,60.27,60.37,64.61$, $77.45,116.58,117.67,125.53,127.68,131.50,135.08,140.00,141.33,169.35$; HRMS (APCI) $\mathrm{m} / \mathrm{z}$ $\left([\mathrm{M}+\mathrm{H}]^{+}\right)$calcd for $\mathrm{C}_{21} \mathrm{H}_{22} \mathrm{BrN}_{2} \mathrm{O}_{2}: 413.0859$, found: 413.0845 .

$\left[{ }^{1} \mathrm{H}\right.$ NMR spectrum of the crude mixture]

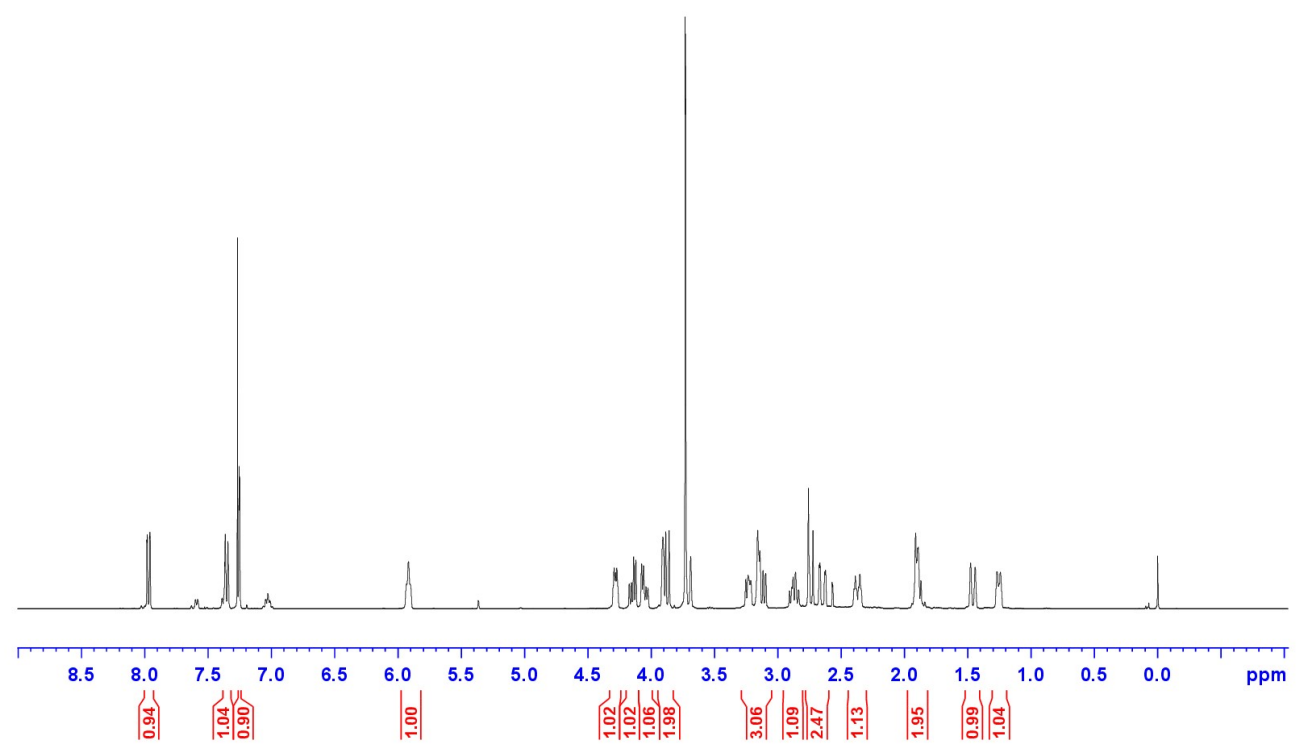

\section{2,4-Dibromostrychnidin-10-one $\left(1 \mathrm{y}-\mathrm{Br}_{2}\right)$}<smiles></smiles>

(240 mg, 98\% yield), white solid, single crystals suitable for X-ray analysis were obtained by pentane vapor diffusion into THF solution, m.p. $180{ }^{\circ} \mathrm{C}$ (decompose); ${ }^{1} \mathrm{H}$ NMR $\left(400 \mathrm{MHz}, \mathrm{CDCl}_{3}\right) \delta 1.20$ (dt, $J=10.6$, $2.4 \mathrm{~Hz}, 1 \mathrm{H}), 1.48(\mathrm{~d}, J=14.4 \mathrm{~Hz}, 1 \mathrm{H}), 1.67-1.74(\mathrm{~m}, 1 \mathrm{H}), 1.81-1.86(\mathrm{~m}, 1 \mathrm{H}), 2.38(\mathrm{dt}, J=14.4,4.2 \mathrm{~Hz}$, 1H), 2.64-2.72 (m, 2H), 2.80-2.88 (m, 1H), 3.08-3.18 (m, 3H), 3.67 (dd, $J=14.9,1.3 \mathrm{~Hz}, 1 \mathrm{H}), 3.92(\mathrm{~s}, 1 \mathrm{H})$, 4.03-4.08 (m, 1H), 4.13-4.19 (m, 1H), 4.28-4.33 (m, 1H), $5.90(\mathrm{t}, J=6.0 \mathrm{~Hz}, 1 \mathrm{H}), 7.18(\mathrm{~d}, J=1.8 \mathrm{~Hz}, 1 \mathrm{H})$, 
$7.63(\mathrm{~d}, J=1.8 \mathrm{~Hz}, 1 \mathrm{H}) ;{ }^{13} \mathrm{C} \mathrm{NMR}\left(100 \mathrm{MHz}, \mathrm{CDCl}_{3}\right) \delta 26.82,31.97,43.37,44.48,48.73,49.76,52.53$, $52.56,59.67,61.71,64.72,77.88,111.55,117.50,124.03,127.22,136.11,138.88,140.31,140.71,168.85$; HRMS (APCI) $m / z\left([\mathrm{M}+\mathrm{H}]^{+}\right)$calcd for $\mathrm{C}_{21} \mathrm{H}_{21} \mathrm{Br}_{2} \mathrm{~N}_{2} \mathrm{O}_{2}: 490.9964$, found: 490.9974.

$\left[{ }^{1} \mathrm{H}\right.$ NMR spectrum of the crude mixture $]$

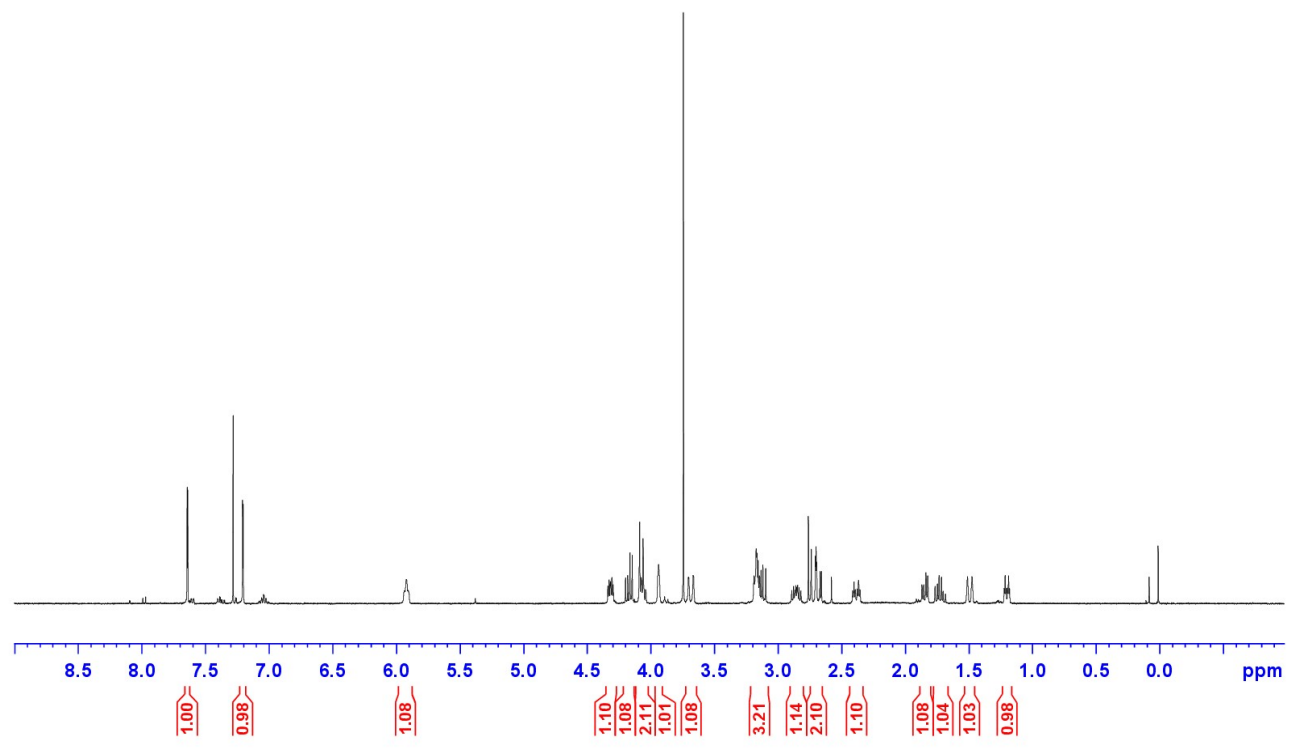




\section{3-7. Bromination of Trip-SMe (Scheme 6)}

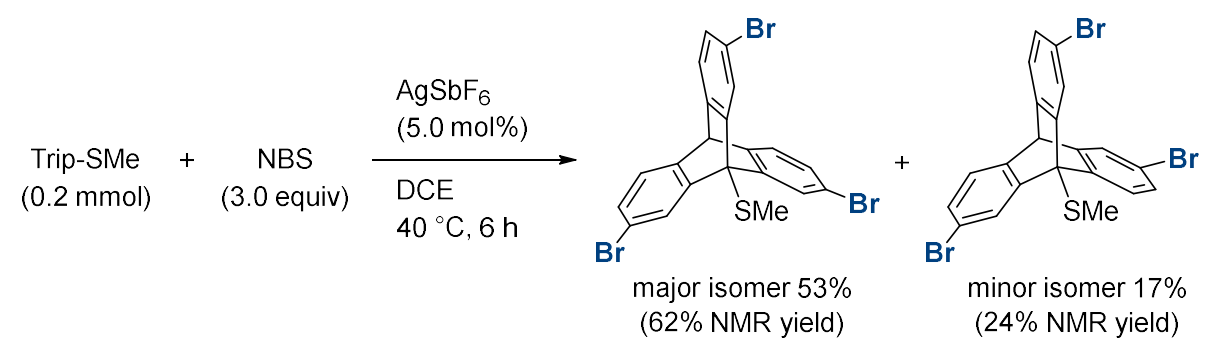

To an oven-dried Schlenk tube was added Trip-SMe (60 mg, $0.2 \mathrm{mmol}$ ), $\mathrm{AgSbF}_{6}(3.4 \mathrm{mg}, 5.0 \mathrm{~mol} \%$ ), and DCE $(2.0 \mathrm{~mL})$. NBS $(107 \mathrm{mg}, 0.6 \mathrm{mmol})$ was added in one portion, and the mixture was stirred at room temperature for $6 \mathrm{~h}$. The resulting suspension was poured into $\mathrm{NaHCO}_{3} \mathrm{aq}$ and extracted with $\mathrm{CHCl}_{3}$ three times. The combined organic layer was dried over $\mathrm{Na}_{2} \mathrm{SO}_{4}$ and concentrated in vacuo. The crude material was subjected to silica gel chromatography (eluent: hexane/EtOAc $=20 / 1$ ) to give the major isomer in 53\% $(57 \mathrm{mg})$. The minor isomer was isolated in 17\% yield $(18 \mathrm{mg})$ by GPC (EtOAc). Single crystals of the major isomer suitable for X-ray analysis were obtained from benzene solution layered with hexane.

\section{2,7,15-Tribromo-Trip-SMe (major isomer)}

white solid, m.p. $220-221{ }^{\circ} \mathrm{C} ;{ }^{1} \mathrm{H}$ NMR (400 MHz, $\left.\mathrm{CDCl}_{3}\right) \delta 2.56$ (s, 3H), 5.28 (s, 1H), 7.19 (dd, $J=7.8$, $1.8 \mathrm{~Hz}, 3 \mathrm{H}), 7.24(\mathrm{~d}, J=7.8 \mathrm{~Hz}, 3 \mathrm{H}), 7.71(\mathrm{~d}, J=1.8 \mathrm{~Hz}, 3 \mathrm{H}) ;{ }^{13} \mathrm{C} \mathrm{NMR}\left(100 \mathrm{MHz}, \mathrm{CDCl}_{3}\right) \delta 14.20,52.47$, 61.11, 119.42, 125.13, 126.98, 129.00, 144.36, 145.58; HRMS (APCI) $m / z\left([\mathrm{M}+\mathrm{H}]^{+}\right)$calcd for $\mathrm{C}_{21} \mathrm{H}_{14} \mathrm{Br}_{3} \mathrm{~S}$ : 533.8361, found: 534.8367 .

\section{2,6,15-Tribromo-Trip-SMe (minor isomer)}

white solid; m.p. $147-148{ }^{\circ} \mathrm{C}$; ${ }^{1} \mathrm{H}$ NMR $\left(400 \mathrm{MHz}, \mathrm{CDCl}_{3}\right) \delta 2.54(\mathrm{~s}, 3 \mathrm{H}), 5.25(\mathrm{~s}, 1 \mathrm{H}), 7.17-7.25(\mathrm{~m}, 5 \mathrm{H})$, $7.44(\mathrm{~d}, J=8.1 \mathrm{~Hz}, 1 \mathrm{H}), 7.52(\mathrm{~d}, J=1.8 \mathrm{~Hz}, 1 \mathrm{H}), 7.70(\mathrm{~d}, J=1.6 \mathrm{~Hz}, 2 \mathrm{H}) ;{ }^{13} \mathrm{C} \mathrm{NMR}\left(100 \mathrm{MHz}, \mathrm{CDCl}_{3}\right) \delta$ $14.19,52.56,61.12$, 119.48, 119.81, 125.08, 125.22, 126.89, 126.95, 128.49, 128.97, 142.53, 144.09, 145.78, 147.41; HRMS (EI) $m / z\left([M]^{+}\right)$calcd for $\mathrm{C}_{21} \mathrm{H}_{13} \mathrm{Br}_{3} \mathrm{~S}$ : 533.8288, found: 533.8284 . 


\section{3-8. Preparation of [Trip-SMeBr][SbCl 5 Br] 2 (Scheme 8)}

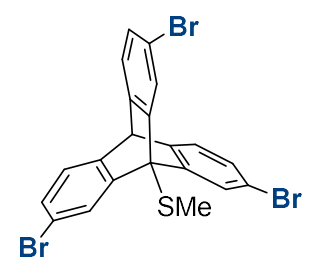

Trip-SMe- $\mathrm{Br}_{3}$

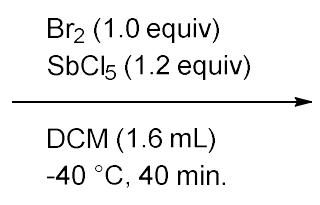

$-40{ }^{\circ} \mathrm{C}, 40 \mathrm{~min}$

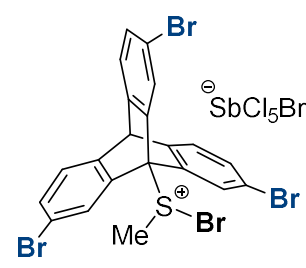

2

To an oven-dried Schlenk tube was added $\mathrm{Br}_{3}$-Trip-SMe $(54 \mathrm{mg}, 0.1 \mathrm{mmol})$ and DCM (1.0 mL). Bromine (16 mg in $0.1 \mathrm{~mL}$ DCM, $0.1 \mathrm{mmol}$ ) was added dropwise at $-40{ }^{\circ} \mathrm{C}$, and the resulting orange solution was stirred at this temperature for 10 minutes. $\mathrm{SbCl}_{5}(36 \mathrm{mg}$ in $0.5 \mathrm{~mL} \mathrm{DCM}, 0.12 \mathrm{mmol})$ was then added, and the mixture was stirred for another 30 minutes to give a yellow suspension. Pentane $(3.0 \mathrm{~mL})$ was added to the tube keeping the temperature below $-40{ }^{\circ} \mathrm{C}$. The pale yellow precipitate was collected, washed with cold pentane $\left(-40^{\circ} \mathrm{C}\right)$ three times, and dried in high vacuum. The product was transferred to a vial in glove box and stored in fridge ( $74 \mathrm{mg}, 75 \%$ yield). Single crystals suitable for X-ray diffraction analysis were obtained from DCM solution layered with pentane below $-30{ }^{\circ} \mathrm{C}$. NMR spectra were measured at $-50{ }^{\circ} \mathrm{C}$ because of the low stability in solution. This compound was not much soluble at the temperature and gave broad signals, so that two peaks (SMe and one quaternary benzene carbon) in the ${ }^{13} \mathrm{C}$ NMR spectrum were not observed.

${ }^{1} \mathrm{H}$ NMR $\left(400 \mathrm{MHz}, \mathrm{CD}_{2} \mathrm{Cl}_{2},-50{ }^{\circ} \mathrm{C}\right) \delta 3.58(\mathrm{~s}, 3 \mathrm{H}), 5.48(\mathrm{~s}, 1 \mathrm{H}), 7.08-7.36(\mathrm{~m}, 9 \mathrm{H}) ;{ }^{13} \mathrm{C} \mathrm{NMR}(400 \mathrm{MHz}$, $\left.\mathrm{CD}_{2} \mathrm{Cl}_{2},-50{ }^{\circ} \mathrm{C}\right) \delta 51.16,58.19,118.56,125.26,126.75,130.01,143.11$. 
${ }^{1} \mathrm{H}$ NMR (2)

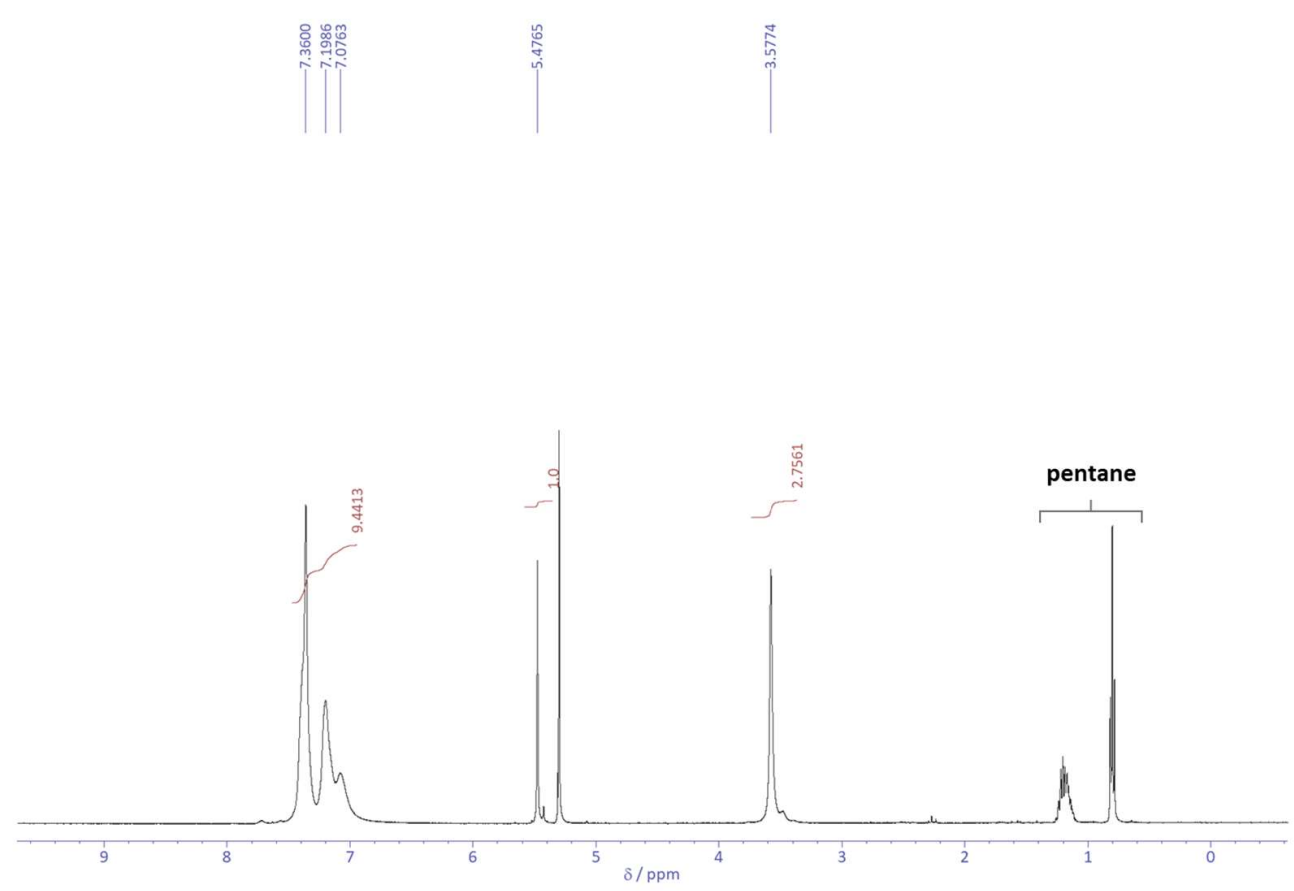

${ }^{13} \mathrm{C}$ NMR (2)

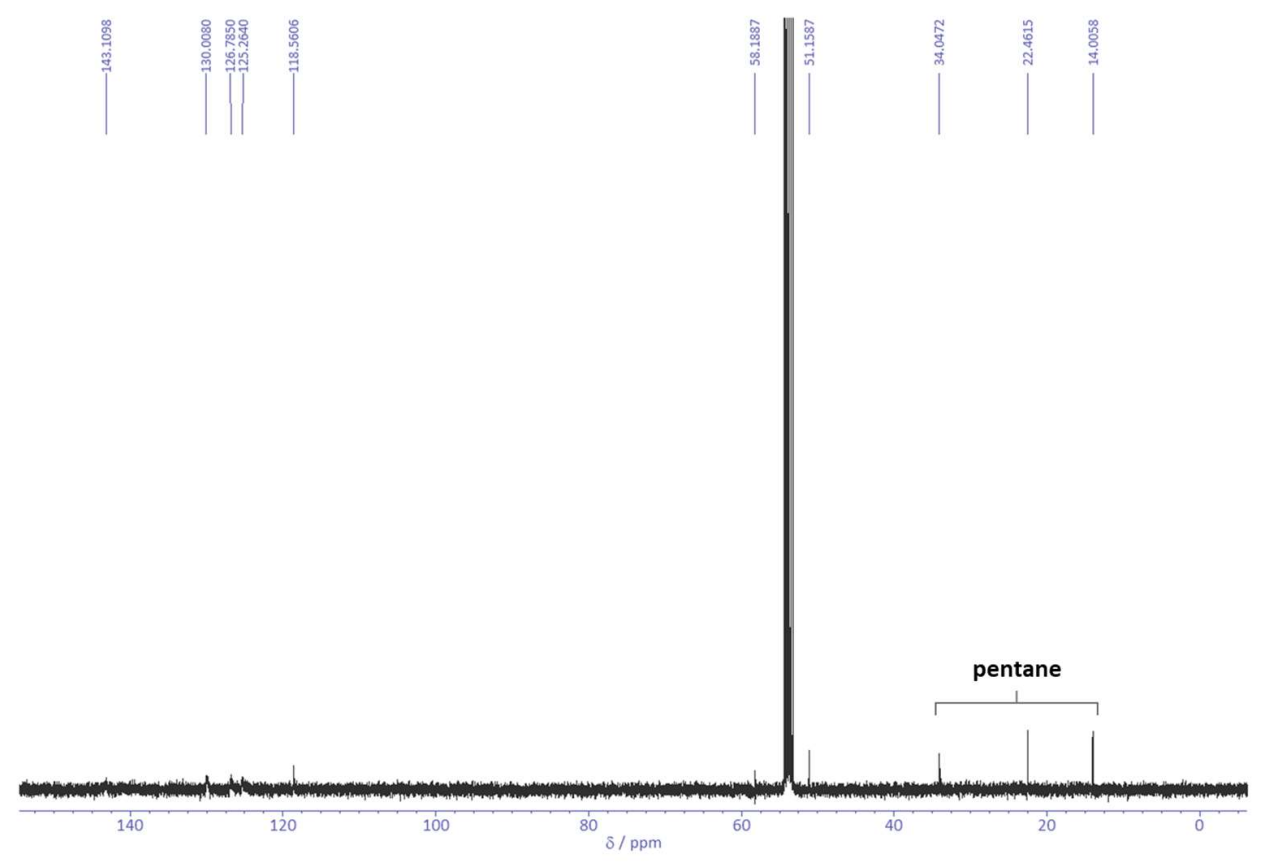




\section{X-ray Crystallography}

The structures were refined on $\mathrm{F}^{\wedge} 2$ by full-matrix least-squares method, using SHELXL-2016/6. ${ }^{30}$ Hydrogen atoms were included in the refinement on calculated positions riding on their carrier atoms. ORTEP- $3^{31}$ programs were used to draw the molecules. The CCDC numbers are 1939787 (Trip-SMe) $1939788\left(\mathbf{1 b}-\mathrm{Cl}_{4}\right), 1967150$ (1h-Br4), 1939789 (1y-Br $), 1939790$ (Trip-SMe-Br $)$ ), and 1939791 (2). These data can be obtained free of charge from the Cambridge Crystallographic Data Centre via www.ccdc.cam.ac.uk/data_request/cif.

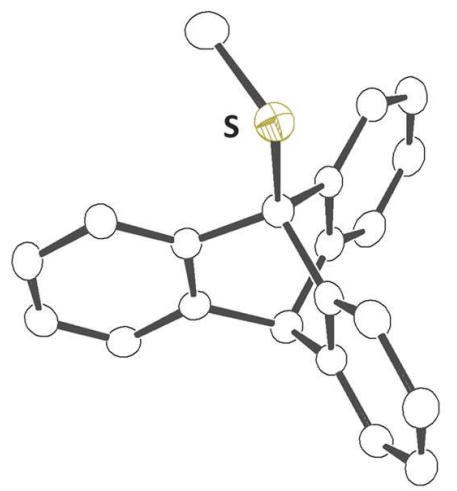

Figure S1. ORTEP drawing of 1 with 50\% thermal probability. Hydrogen atoms are omitted for clarity.

Table S3. Crystal data and collection parameters for Trip-SMe

Empirical formula

Crystal system

Space group

Unit cell parameter $[\AA]$

Cell volume $\left[\AA^{3}\right]$

Z

Crystal size $[\mathrm{mm}]$

$R$ factor $(I>2.0 \sigma(I))$

$R$ factor (all data)

Goodness of fit on $F^{2}$

The number of unique reflections
C21 H16 S

orthorhombic

Pbca (No. 61)

$\mathrm{a}=15.147(15)$

$\mathrm{b}=13.556(13)$

$\mathrm{c}=15.130(15)$

$3107(5)$

8

$0.230 \times 0.130 \times 0.120$

$R 1=0.0760, w R 2=0.1612$

$R 1=0.1247, w R 2=0.1870$

1.092

$3558\left(R_{\text {int }}=0.1410\right)$ 


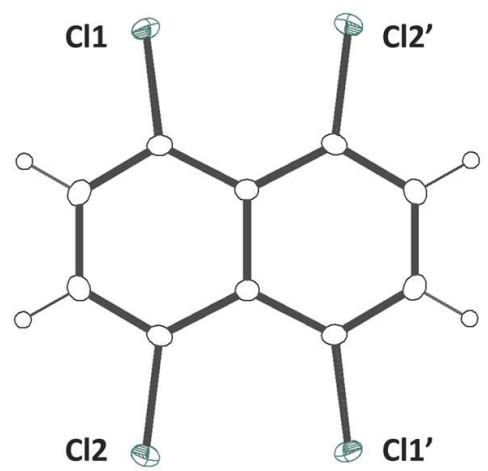

Figure S2. ORTEP drawing of $\mathbf{1 b}-\mathrm{Cl}_{4}$ with $50 \%$ thermal probability.

Table S4. Crystal data and collection parameters for $\mathbf{1 b}-\mathrm{Cl}_{4}$

Empirical formula

Crystal system

Space group

Unit cell parameter $[\AA]$

Cell volume $\left[\AA^{3}\right]$

$\mathrm{Z}$

$R$ factor $(I>2.0 \sigma(I))$

$R$ factor (all data)

Goodness of fit on $F^{2}$

The number of unique reflections
$\mathrm{C} 5 \mathrm{H} 2 \mathrm{Cl} 2$

orthorhombic

Pccn (No. 56)

$\mathrm{a}=14.9360(12)$

$\mathrm{b}=9.3777(9)$

$\mathrm{c}=6.9535(7)$

973.94(16)

8

$R 1=0.0313, w R 2=0.0828$

$R 1=0.0364, w R 2=0.0860$

1.080

$1234\left(R_{\text {int }}=0.0466\right)$ 


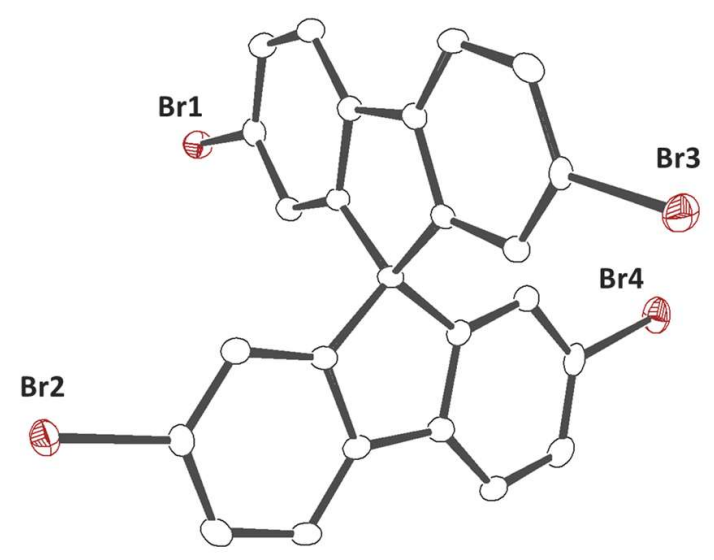

Figure S3. ORTEP drawing of $\mathbf{1 h}-\mathrm{Br}_{4}$ with $50 \%$ thermal probability. Hydrogen atoms are omitted for clarity.

Table S5. Crystal data and collection parameters for $\mathbf{1 h}-\mathrm{Br}_{4}$

Empirical formula

Crystal system

Space group

Unit cell parameter $[\AA, \operatorname{deg}]$

Cell volume $\left[\AA^{3}\right]$

$\mathrm{Z}$

$R$ factor $(I>2.0 \sigma(I))$

$R$ factor (all data)

Goodness of fit on $F^{2}$

The number of unique reflections
$\mathrm{C} 25 \mathrm{H} 12 \mathrm{Br} 4,2(\mathrm{C} \mathrm{H} \mathrm{Cl} 3)$

triclinic

$P$-1 (No. 2)

$\mathrm{a}=10.1187(2), \alpha=83.594(2)$

$\mathrm{b}=10.8745(3), \beta=89.665(2)$

$\mathrm{c}=14.7135(3), \gamma=62.659(2)$

$1427.28(6)$

2

$R 1=0.0279, w R 2=0.0598$

$R 1=0.0372, w R 2=0.0620$

1.035

$7081\left(R_{\text {int }}=0.0291\right)$ 


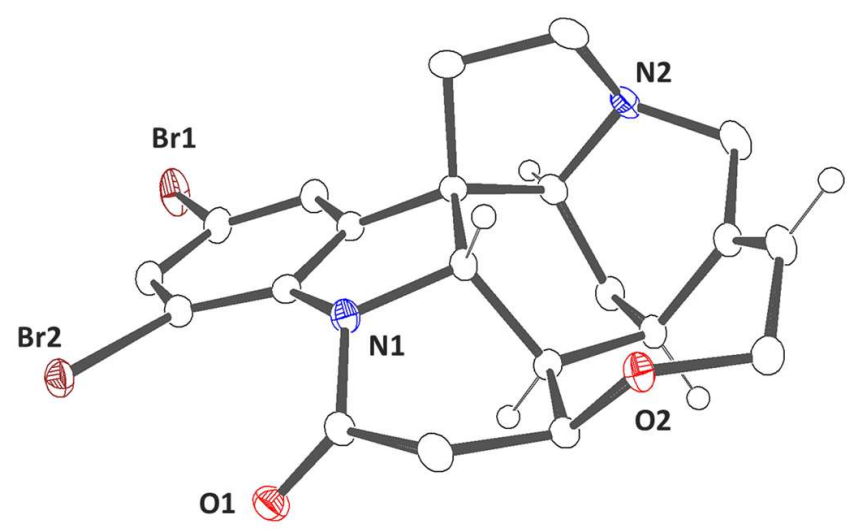

Figure S4. ORTEP drawing of $\mathbf{1 y}-\mathrm{Br}_{2}$ with $40 \%$ thermal probability. Hydrogen atoms except on vinylic and tertiary carbons as well as solvent molecules were omitted for clarity.

Table S6. Crystal data and collection parameters for $\mathbf{1 y - \mathrm { Br } _ { 2 }}$

Empirical formula

Crystal system

Space group

Unit cell parameter $[\AA$, deg $]$

Cell volume $\left[\AA^{3}\right]$

$\mathrm{Z}$

$R$ factor $(I>2.0 \sigma(I))$

$R$ factor (all data)

Goodness of fit on $F^{2}$

The number of unique reflections
$\mathrm{C} 21 \mathrm{H} 20 \mathrm{Br} 2 \mathrm{~N} 2 \mathrm{O} 2$

hexagonal

$P 6_{1}$ (No. 169)

$\mathrm{a}=17.12740(10)$

$\mathrm{b}=17.12740(10)$

$\mathrm{c}=11.72910(10), \gamma=120$

2979.74(4)

6

$R 1=0.0485, w R 2=0.1195$

$R 1=0.0486, w R 2=0.1198$

1.038

$3985\left(R_{\text {int }}=0.0480\right)$ 


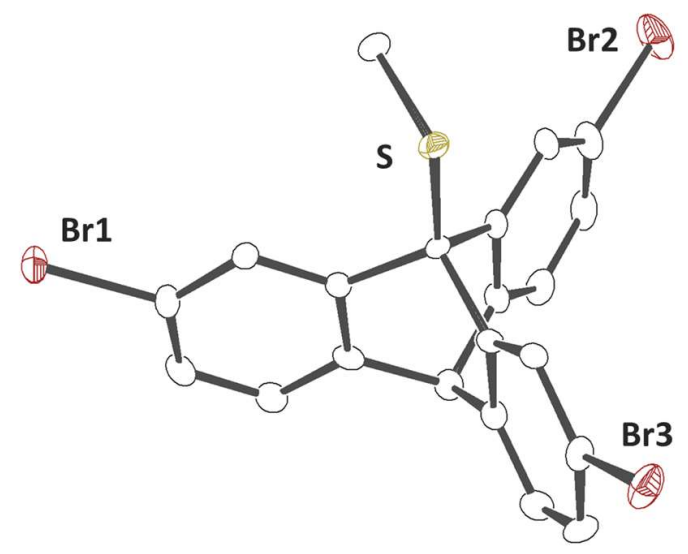

Figure S5. ORTEP drawing of Trip-SMe- $\mathrm{Br}_{3}$ with $50 \%$ thermal probability. Hydrogen atoms are omitted for clarity.

Table S7. Crystal data and collection parameters for Trip-SMe-Br 3

Empirical formula

Crystal system

Space group

Unit cell parameter $[\AA$, deg $]$

Cell volume $\left[\AA^{3}\right]$

$\mathrm{Z}$

$R$ factor $(I>2.0 \sigma(I))$

$R$ factor (all data)

Goodness of fit on $F^{2}$

The number of unique reflections
2 (C21 H13 Br3 S)

monoclinic

$P 2_{1} / n$ (No. 14)

$\mathrm{a}=12.6655(5)$

$\mathrm{b}=19.4145(8), \beta=94.624(3)$

$\mathrm{c}=15.7065(5)$

$3849.6(3)$

4

$R 1=0.0460, w R 2=0.1180$

$R 1=0.0654, w R 2=0.1114$

1.053

$9323\left(R_{\text {int }}=0.0589\right)$ 


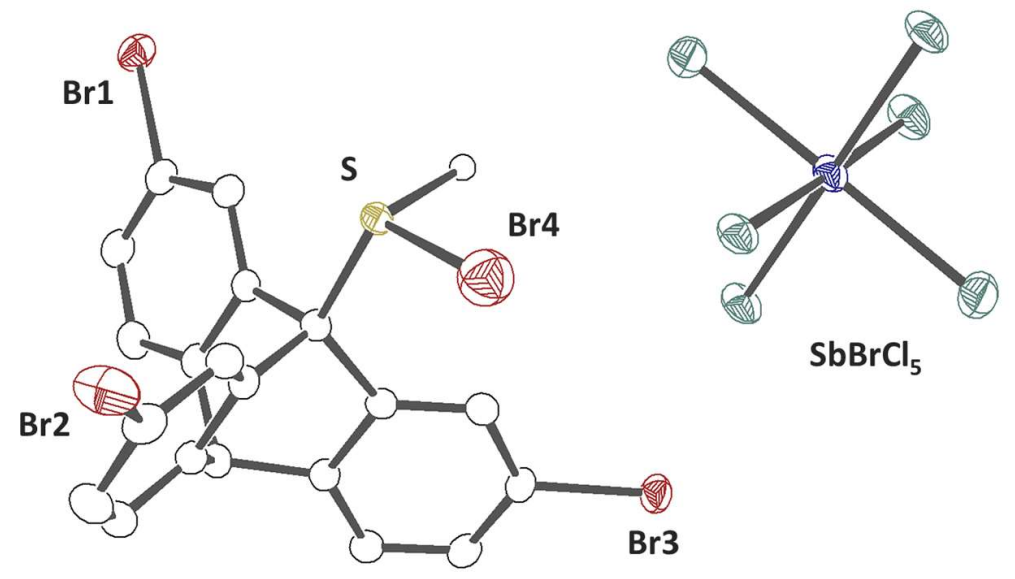

Figure S6. ORTEP drawing of 2 with $40 \%$ thermal probability. Only major part of the disordered structures is shown.

Table S8. Crystal data and collection parameters for complex 2

Empirical formula

Formula weight

Crystal system

Space group

Unit cell parameter $[\AA, \operatorname{deg}]$

Cell volume $\left[\AA^{3}\right]$

$\mathrm{Z}$

$R$ factor $(I>2.0 \sigma(I))$

$R$ factor (all data)

Goodness of fit on $F^{2}$

The number of unique reflections
C21 H10 Br4 C16 S Sb

948.44

monoclinic

$P 2{ }_{1} / m$ (No. 11)

$\mathrm{a}=12.1698(2)$

$\mathrm{b}=22.4948(2), \beta=104.8150(10)$

$\mathrm{c}=15.2237(2)$

4029.05(9)

4

$R 1=0.0541, w R 2=0.1496$

$R 1=0.0594, w R 2=0.1559$

1.038

$8340\left(R_{\text {int }}=0.0666\right)$ 


\section{Kinetic Study}<smiles>Cc1ccc([N+](=O)[O-])cc1</smiles>

[xylene]

[NBS]

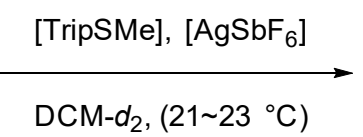

$\mathrm{DCM}-\mathrm{d}_{2},\left(21 \sim 23{ }^{\circ} \mathrm{C}\right)$<smiles>Cc1ccc(C)c(Br)c1</smiles>

[product]<smiles>O=C1CCC(=O)N1</smiles>

[imide]

$\mathrm{AgSbF}_{6}$, Trip-SMe, dibromomethane (internal standard), and DCM- $d_{2}$ were added to a $5 \mathrm{~mm}$ NMR tube in glovebox. The tube was taken out from glovebox, and xylene was added via syringe. Immediately after the addition of NBS, the tube was shaken vigorously to make the mixture into a homogenous solution (here set the time as "zero" in time course) and injected into the NMR magnet. Temperature inside the magnet was kept at $21 \sim 23{ }^{\circ} \mathrm{C}$ during the experiments. Concentrations were calculated from the integration ratio in ${ }^{1} \mathrm{H}$ NMR analyses. For the variable time normalization analysis (VTNA), the concentration of product was plotted versus the time integral of the concentration of each component (xylene, NBS, Trip-SMe, and $\mathrm{AgSbF}_{6}$ ) with an exponent $x$, represented as follows: ${ }^{32}$

$$
\int_{t=0}^{t=n}[\mathrm{~A}]^{x} d t=\sum_{t=1}^{n}\left(\frac{[\mathrm{A}]_{i}+[\mathrm{A}]_{i-1}}{2}\right)^{x}\left(t_{i}-t_{i-1}\right), \quad \text { if }[\mathrm{A}]=\mathrm{constant}, \int_{t=0}^{t=n}[\mathrm{~A}]^{x} d t=t[\mathrm{~A}]^{x}
$$

\section{5-1. Time course experiments}

The concentration of Trip-SMe was calculated from the integration of its benzylic hydrogen in ${ }^{1} \mathrm{H}$ NMR to minimize the effect of equilibria within sulfonium salts.

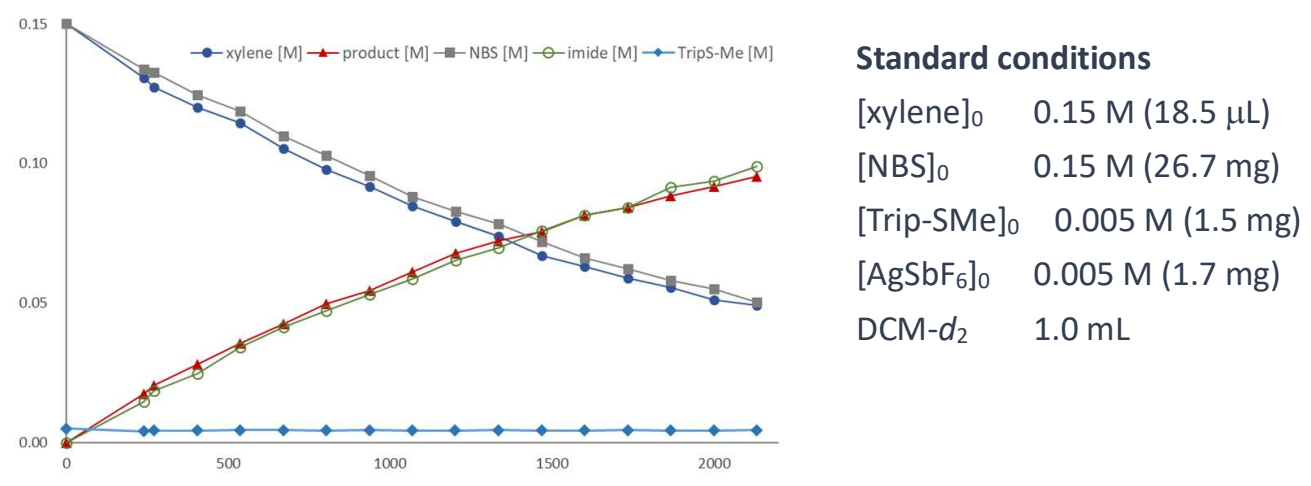



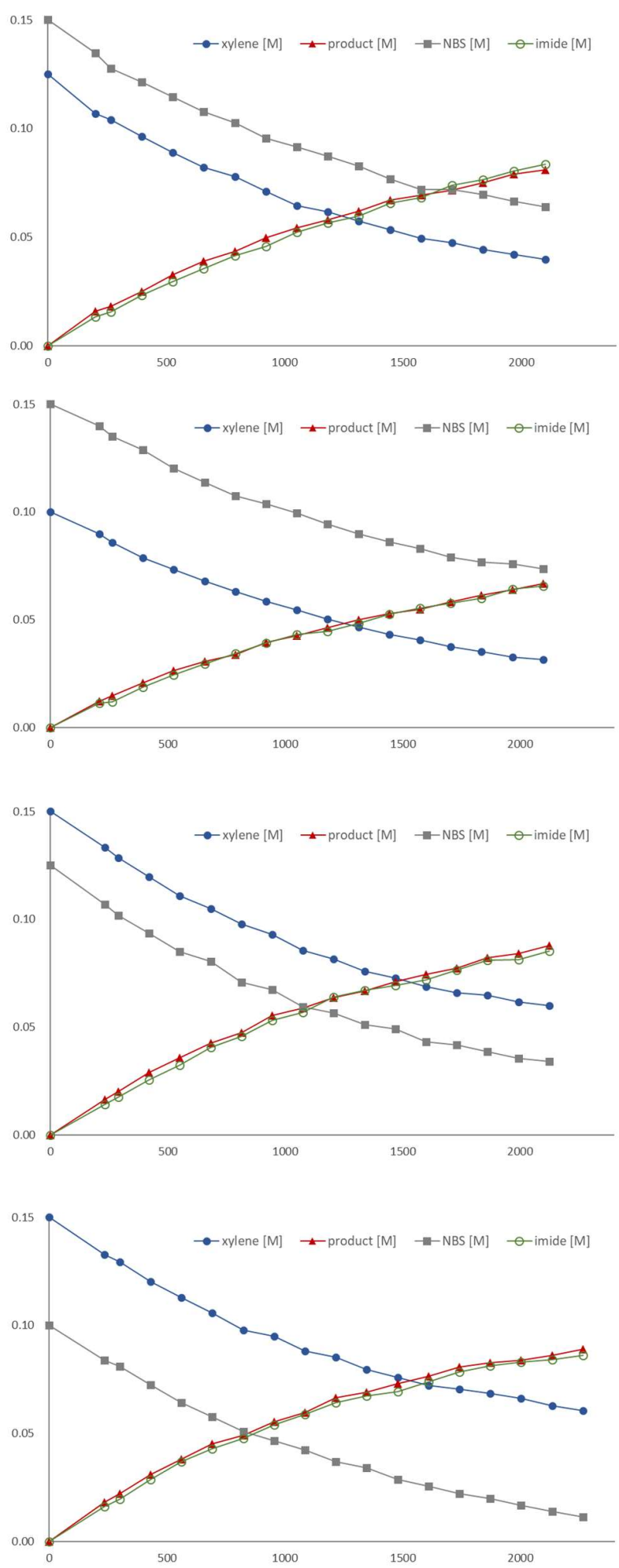

[xylene] $0.125 \mathrm{M}(15.4 \mu \mathrm{L})$

[NBS] $\quad 0.15 \mathrm{M}(26.7 \mathrm{mg})$

[Trip-SMe] $\quad 0.005 \mathrm{M}(1.5 \mathrm{mg})$ $\left[\mathrm{AgSbF}_{6}\right]_{0} \quad 0.005 \mathrm{M}(1.7 \mathrm{mg})$ DCM- $d_{2} \quad 1.0 \mathrm{~mL}$

[xylene $]_{0} \quad 0.10 \mathrm{M}(12.3 \mu \mathrm{L})$

[NBS] $\quad 0.15 \mathrm{M}(26.7 \mathrm{mg})$

[Trip-SMe] $0.005 \mathrm{M}(1.5 \mathrm{mg})$ $\left[\mathrm{AgSbF}_{6}\right]_{0} \quad 0.005 \mathrm{M}(1.7 \mathrm{mg})$ DCM- $d_{2} \quad 1.0 \mathrm{~mL}$

[xylene]o $\quad 0.15 \mathrm{M}(18.5 \mu \mathrm{L})$

$[\mathrm{NBS}]_{0} \quad 0.125 \mathrm{M}(22.3 \mathrm{mg})$ [Trip-SMe] $0.005 \mathrm{M}(1.5 \mathrm{mg})$ $\left[\mathrm{AgSbF}_{6}\right]_{0} \quad 0.005 \mathrm{M}(1.7 \mathrm{mg})$ DCM- $d_{2} \quad 1.0 \mathrm{~mL}$

[xylene] $\quad 0.15 \mathrm{M}(18.5 \mu \mathrm{L})$ $[\mathrm{NBS}]_{0} \quad 0.10 \mathrm{M}(17.8 \mathrm{mg})$ [Trip-SMe] $0.005 \mathrm{M}(1.5 \mathrm{mg})$

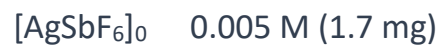
DCM- $d_{2} \quad 1.0 \mathrm{~mL}$ 


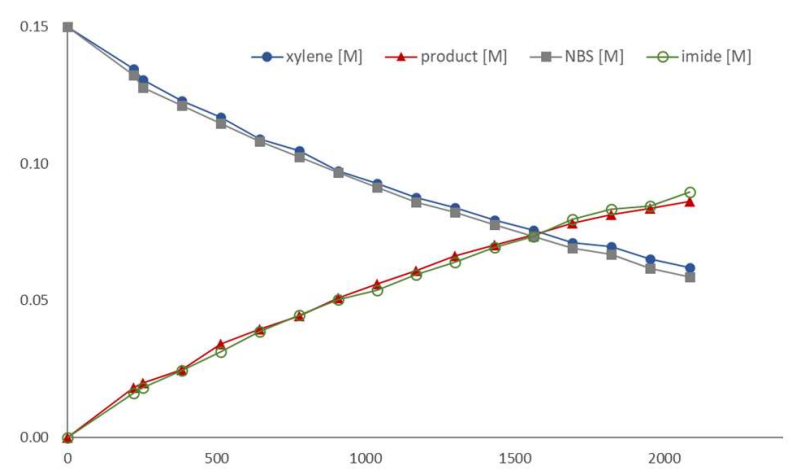

[xylene]o $\quad 0.15 \mathrm{M}(18.5 \mu \mathrm{L})$

$[\mathrm{NBS}]_{0} \quad 0.15 \mathrm{M}(26.7 \mathrm{mg})$

[Trip-SMe] $\quad 0.0075 \mathrm{M}(2.3 \mathrm{mg})$

$\left[\mathrm{AgSbF}_{6}\right]_{0} \quad 0.005 \mathrm{M}(1.7 \mathrm{mg})$

DCM- $d_{2} \quad 1.0 \mathrm{~mL}$

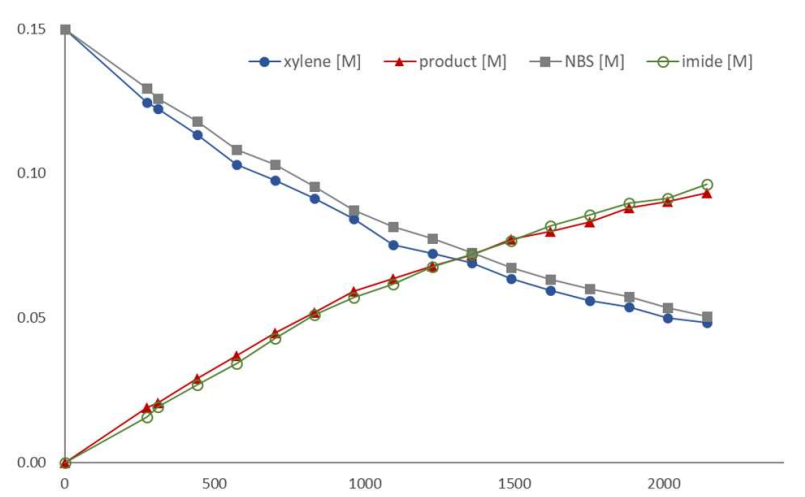

[xylene] $\quad 0.15 \mathrm{M}(18.5 \mu \mathrm{L})$

[NBS] $\quad 0.15 \mathrm{M}(26.7 \mathrm{mg})$

[Trip-SMe] $\quad 0.0025 \mathrm{M}(0.8 \mathrm{mg})$

$\left[\mathrm{AgSbF}_{6}\right]_{0} \quad 0.005 \mathrm{M}(1.7 \mathrm{mg})$

DCM- $d_{2} \quad 1.0 \mathrm{~mL}$

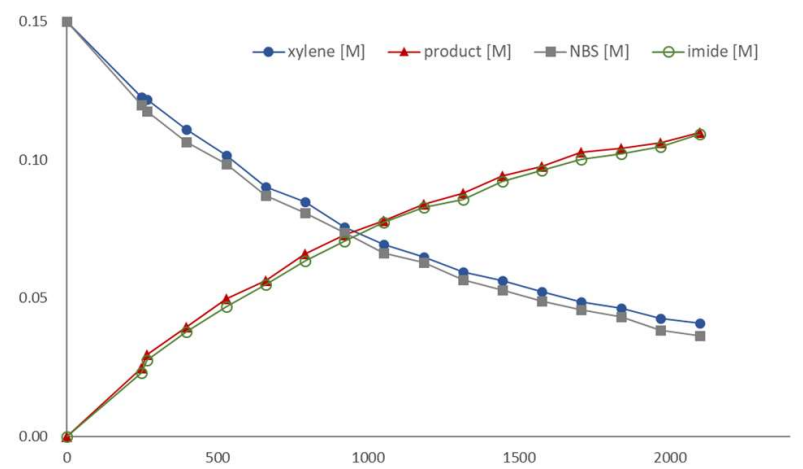

[xylene] $0.15 \mathrm{M}(18.5 \mu \mathrm{L})$

$[\mathrm{NBS}]_{0} \quad 0.15 \mathrm{M}(26.7 \mathrm{mg})$

[Trip-SMe] $\quad 0.005 \mathrm{M}(1.5 \mathrm{mg})$

$\left[\mathrm{AgSbF}_{6}\right]_{0} \quad 0.0075 \mathrm{M}(2.6 \mathrm{mg})$

DCM- $d_{2} \quad 1.0 \mathrm{~mL}$ 


\section{5-2. Variable time normalization analysis}
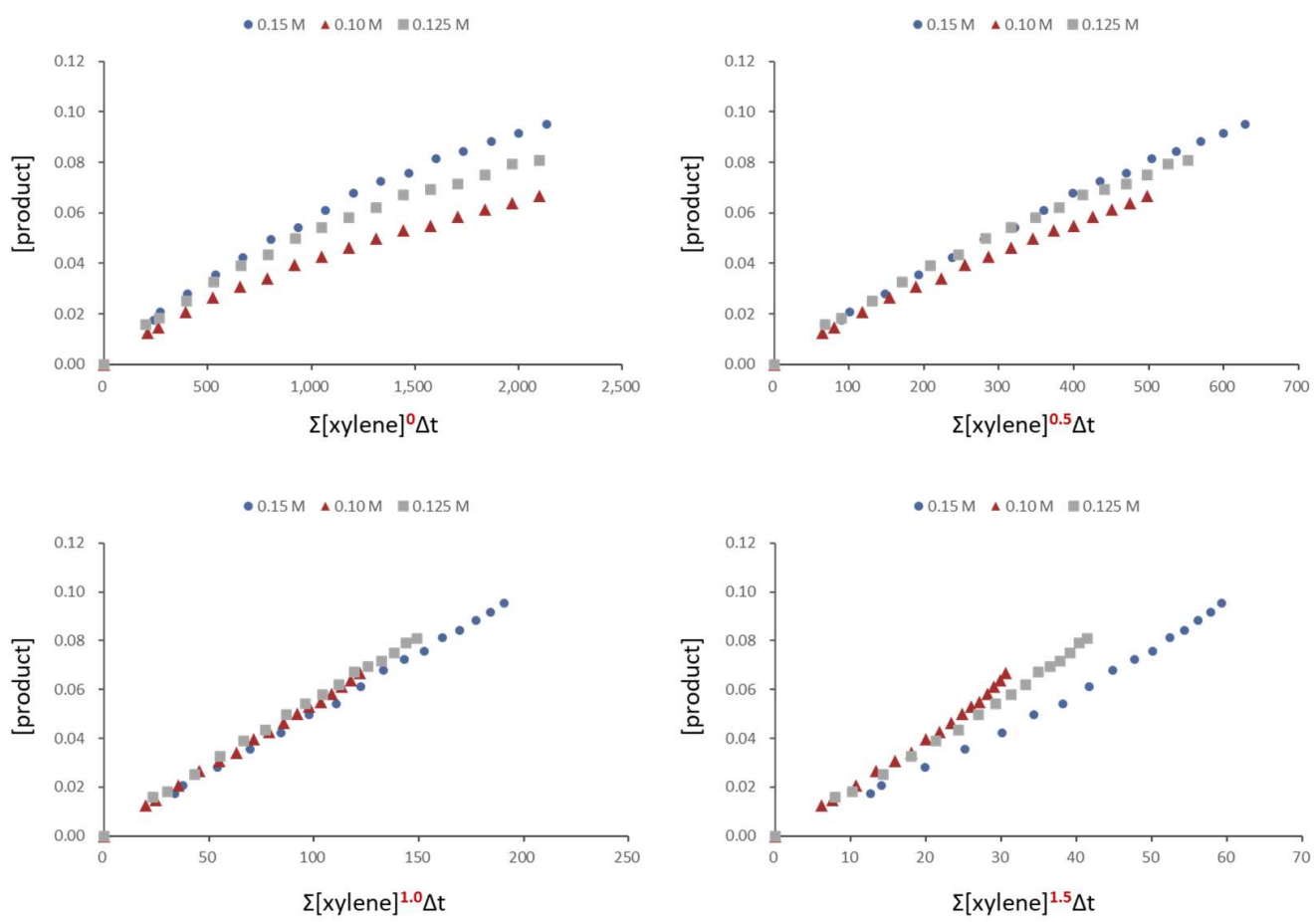

Figure S7. Variable time normalization analysis for the determination of order in xylene.
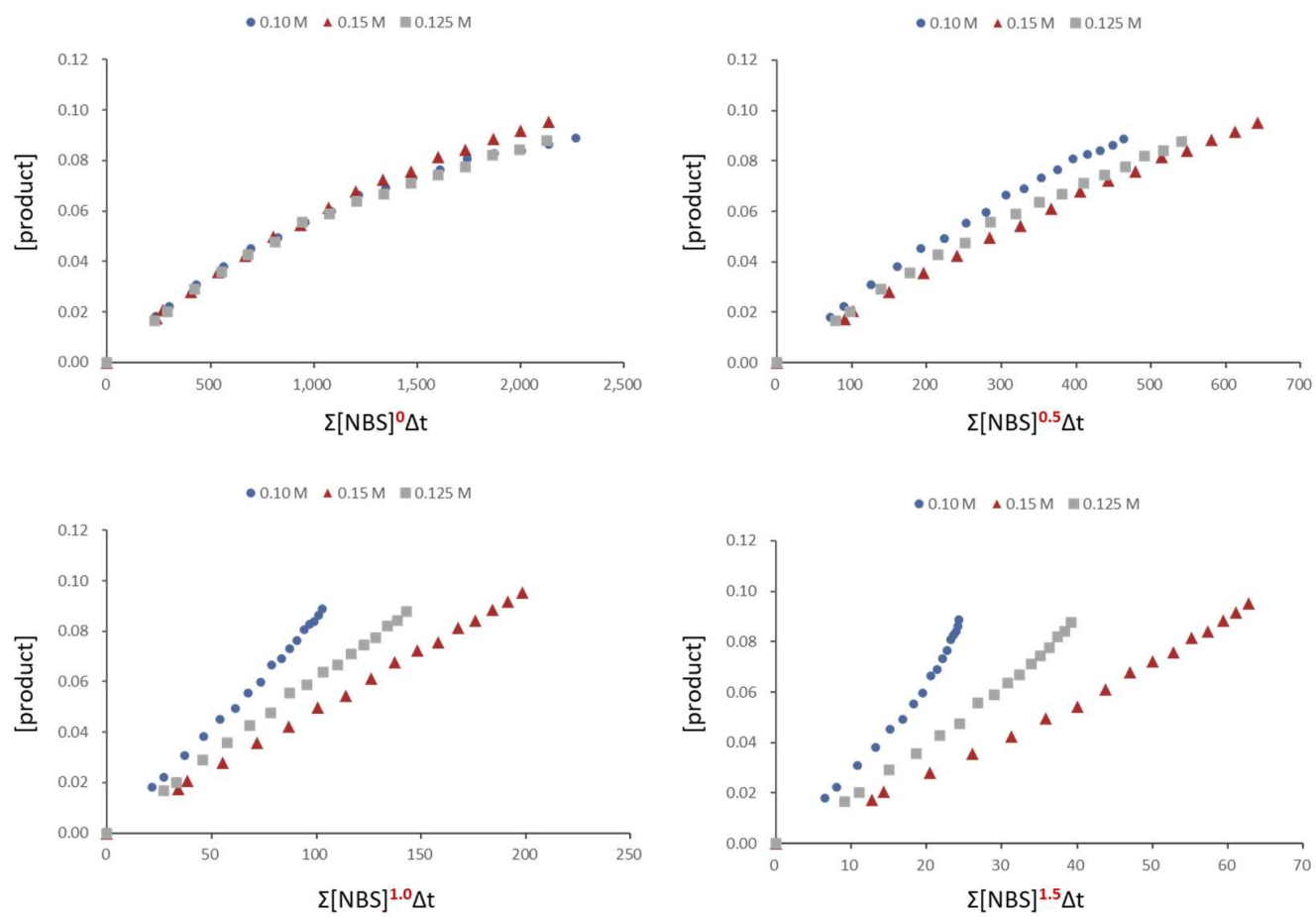

Figure S8. Variable time normalization analysis for the determination of order in NBS. 

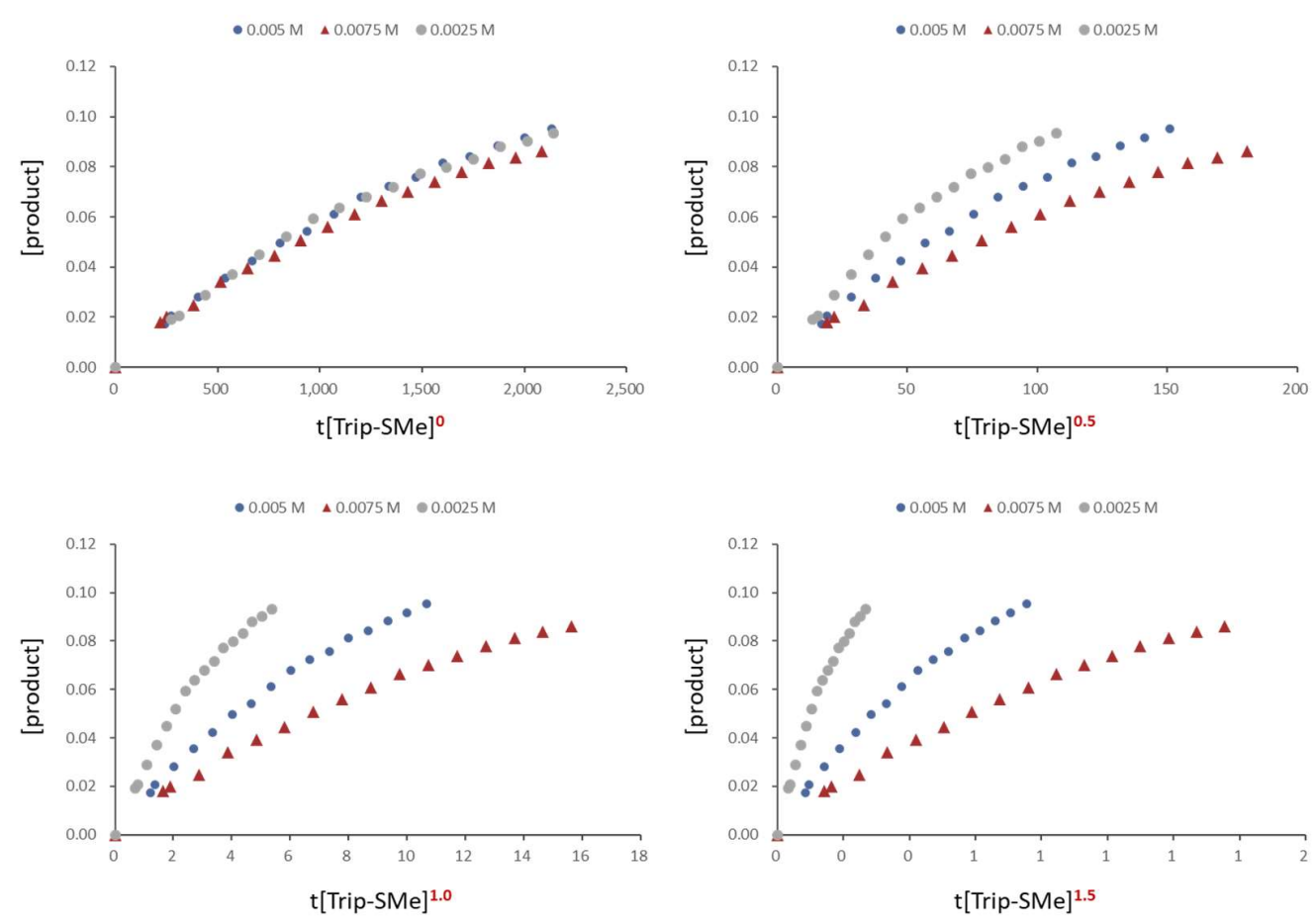

Figure S9. Variable time normalization analysis for the determination of order in Trip-SMe.
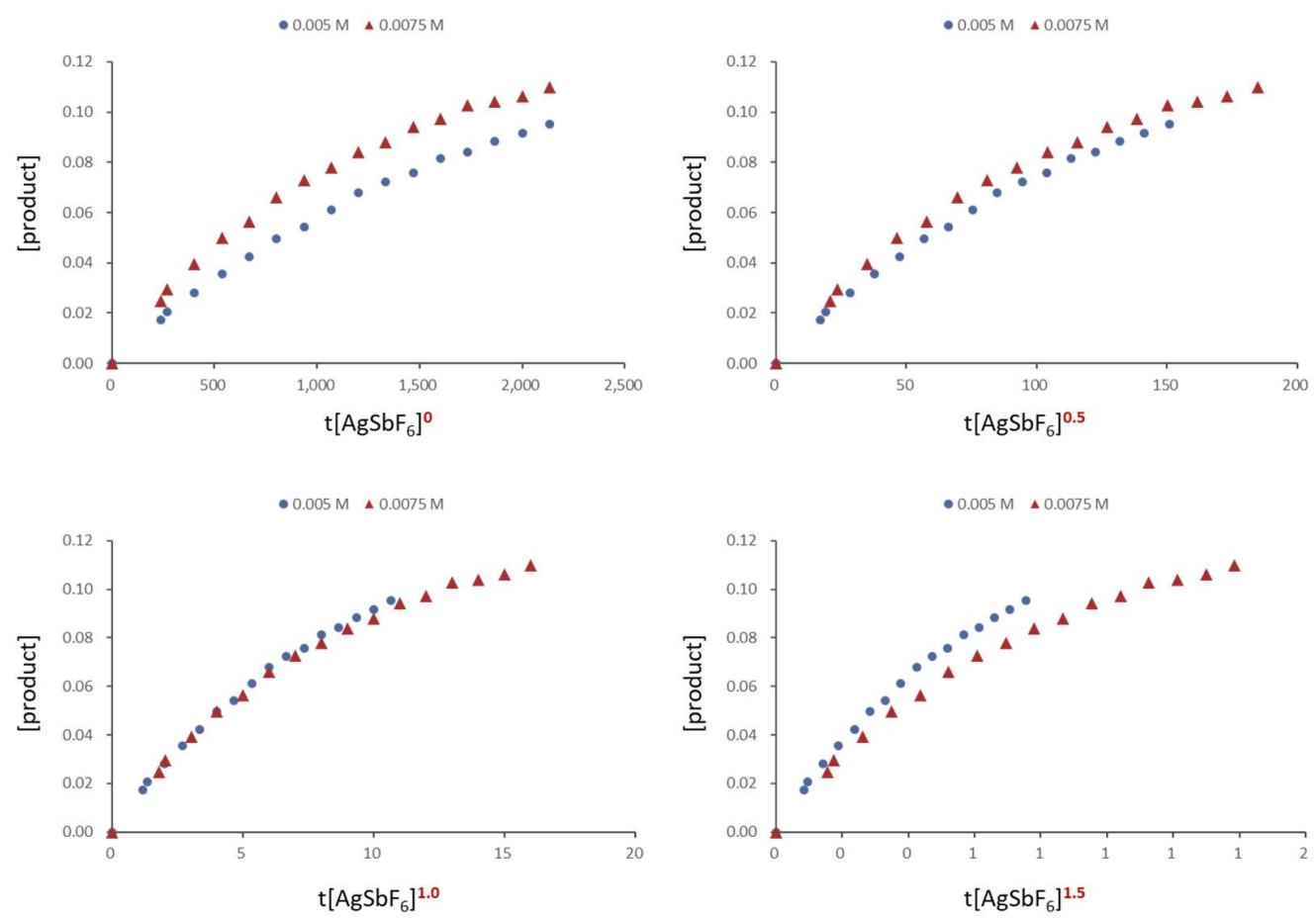

Figure S10. Variable time normalization analysis for the determination of order in $\mathrm{AgSbF}_{6}$. 


\section{5-3. Equilibrium constant measurement}

Trip-SMe (1.5 mg) and NBS $(17.8 \mathrm{mg})$ were dissolved in DCM- $d_{2}(1.0 \mathrm{~mL})$ in a $5 \mathrm{~mm}$ NMR tube. A singlet peak at $2.76 \mathrm{ppm}$, which could be correspond to the methyl group of intermediate $\mathbf{A}$, was observed with a relative integration value of 0.1671 , giving an equilibrium constant of $1.69 \times 10^{-3}$ for the reaction.

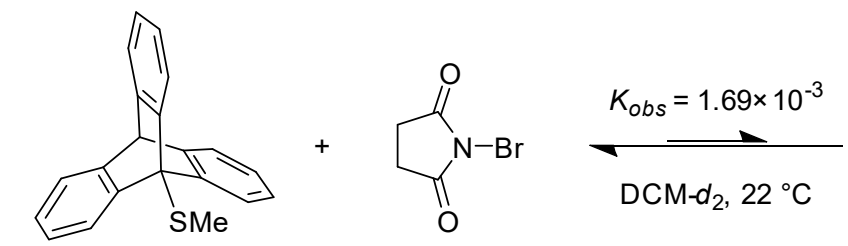

$[\text { Trip-SMe }]_{0}=5.0 \mathrm{mM} \quad[\mathrm{NBS}]_{0}=0.10 \mathrm{M}$

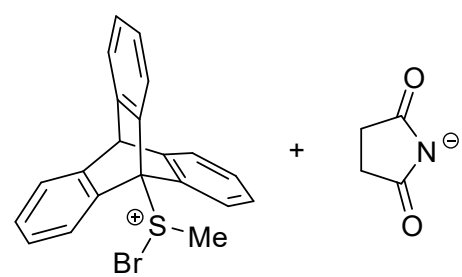

$[\mathrm{A}]=\left[\left(\operatorname{Trip}_{\mathrm{SMeBr}}\right)^{+}\right]=[($imide $)]$

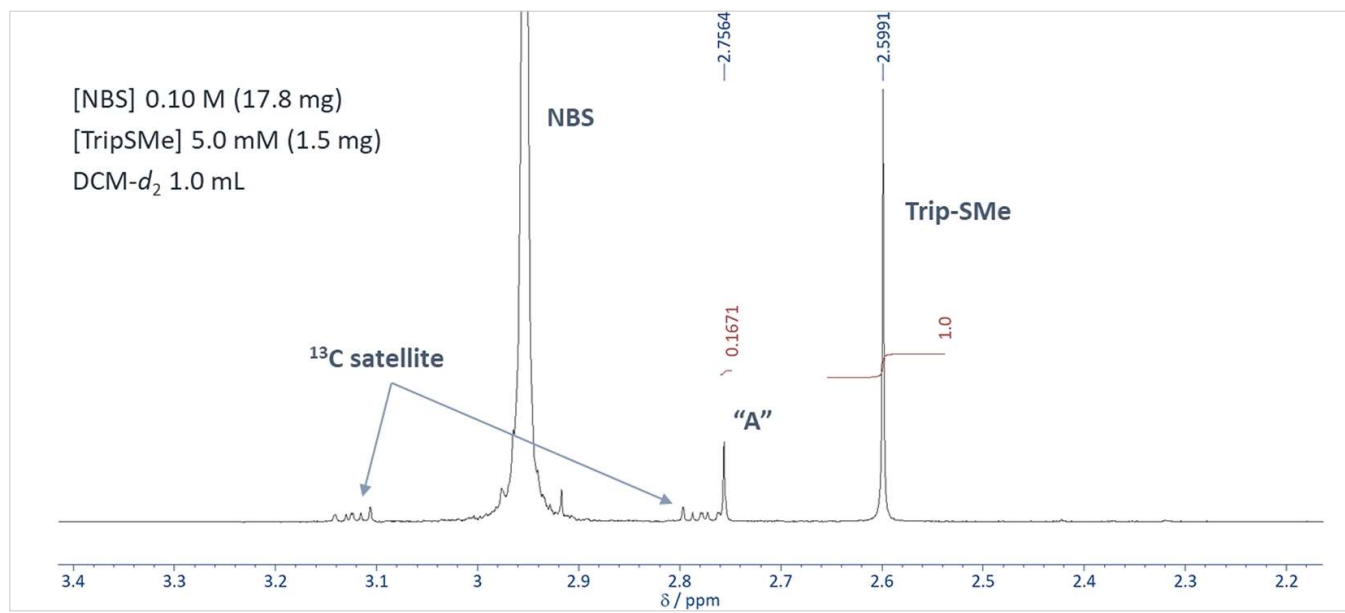

Figure S11. Calculation of an equilibrium constant for the formation of intermediate A. 


\section{Copy of NMR Spectra}

${ }^{1} \mathrm{H}$ NMR (1b-Br 3$)$

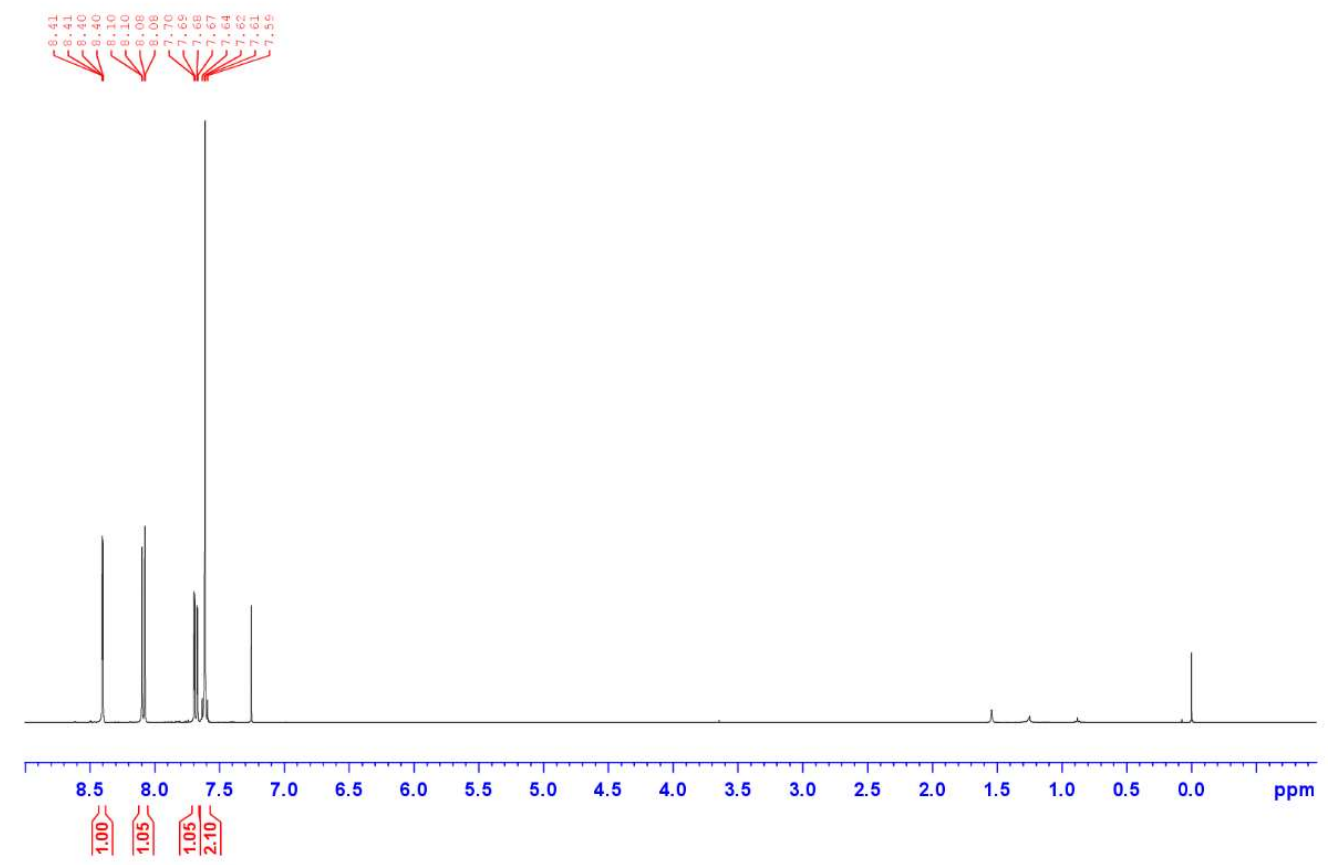

${ }^{13} \mathrm{C}$ NMR $\left(\mathbf{1 b}-\mathrm{Br}_{3}\right)$

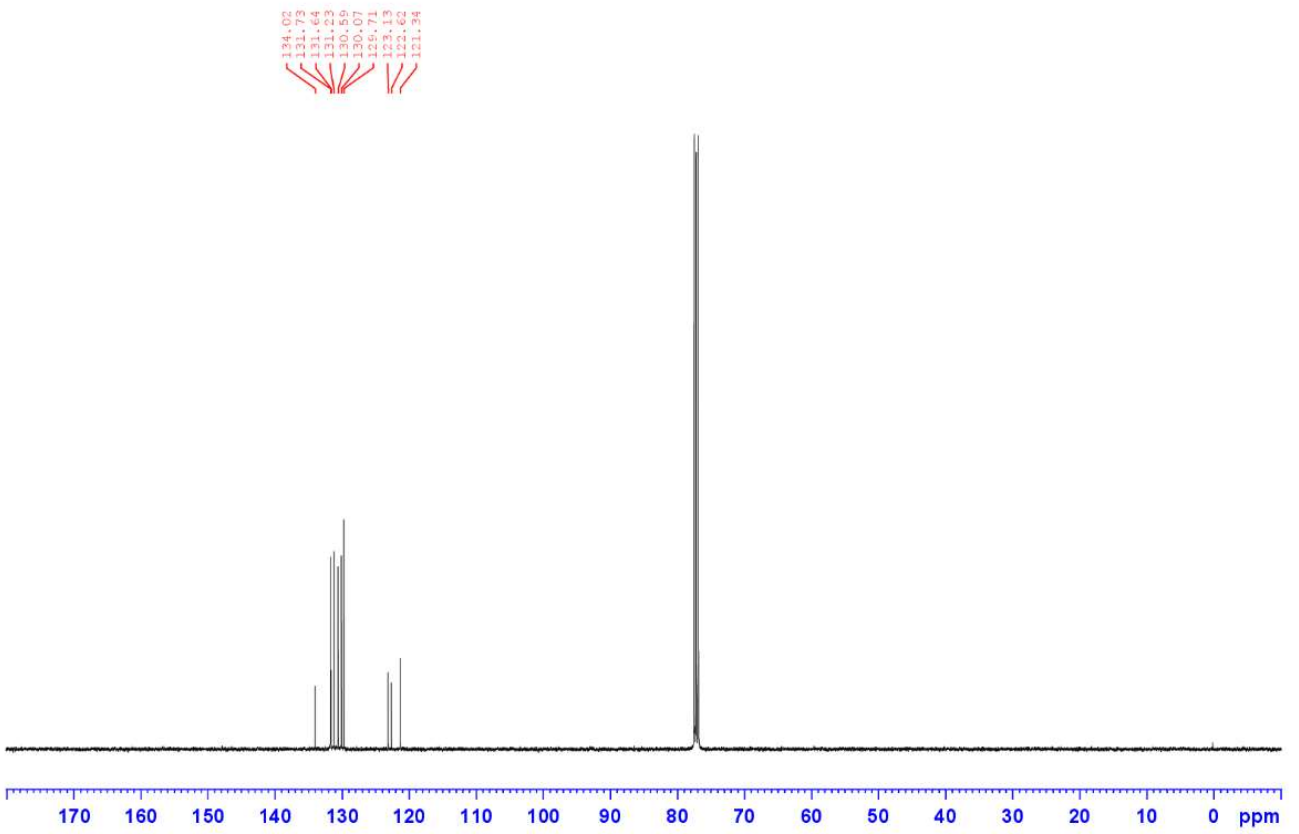


${ }^{1} \mathrm{H}$ NMR $\left(\mathbf{1 b}-\mathrm{Cl}_{4}\right)$

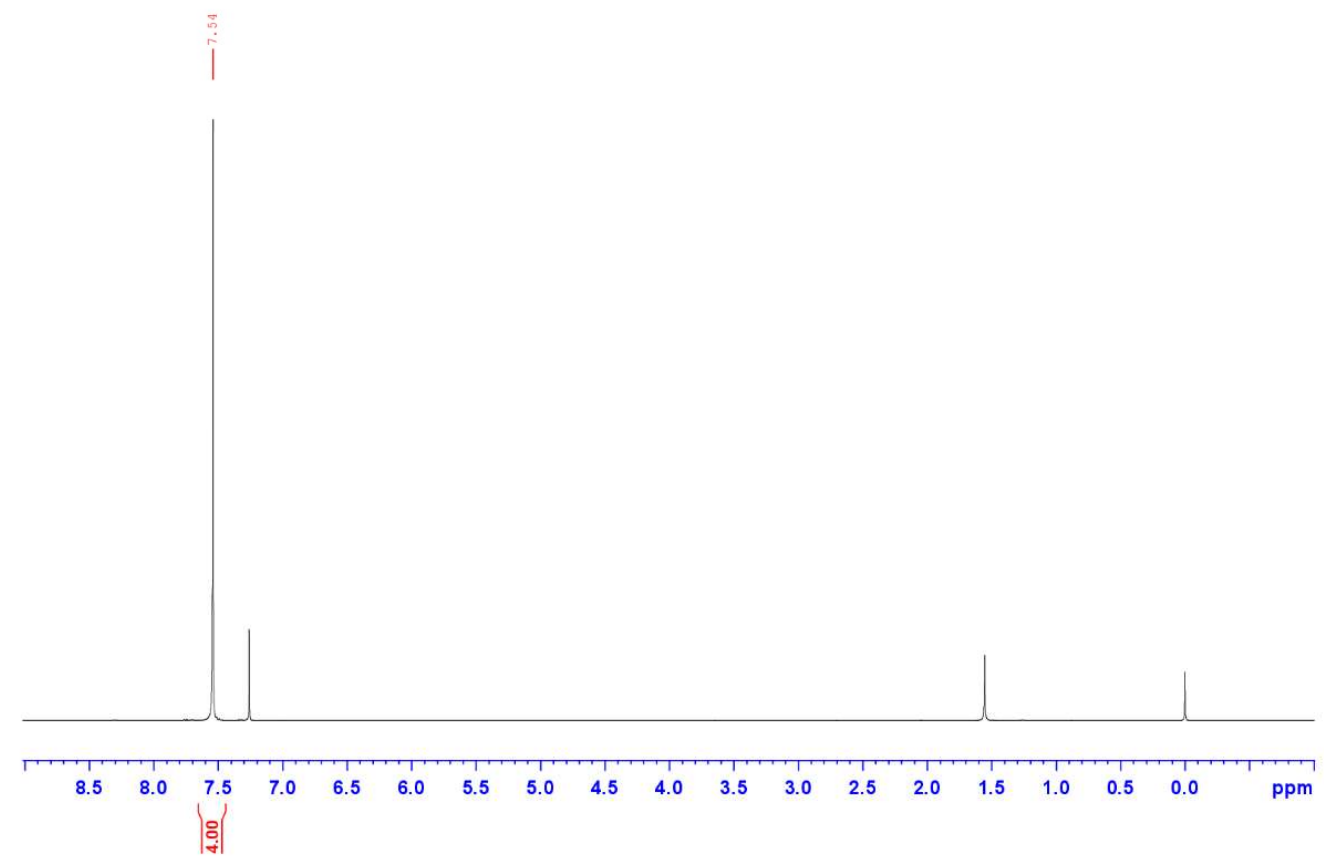

${ }^{13} \mathrm{C}$ NMR $\left(\mathbf{1 b}-\mathrm{Cl}_{4}\right)$

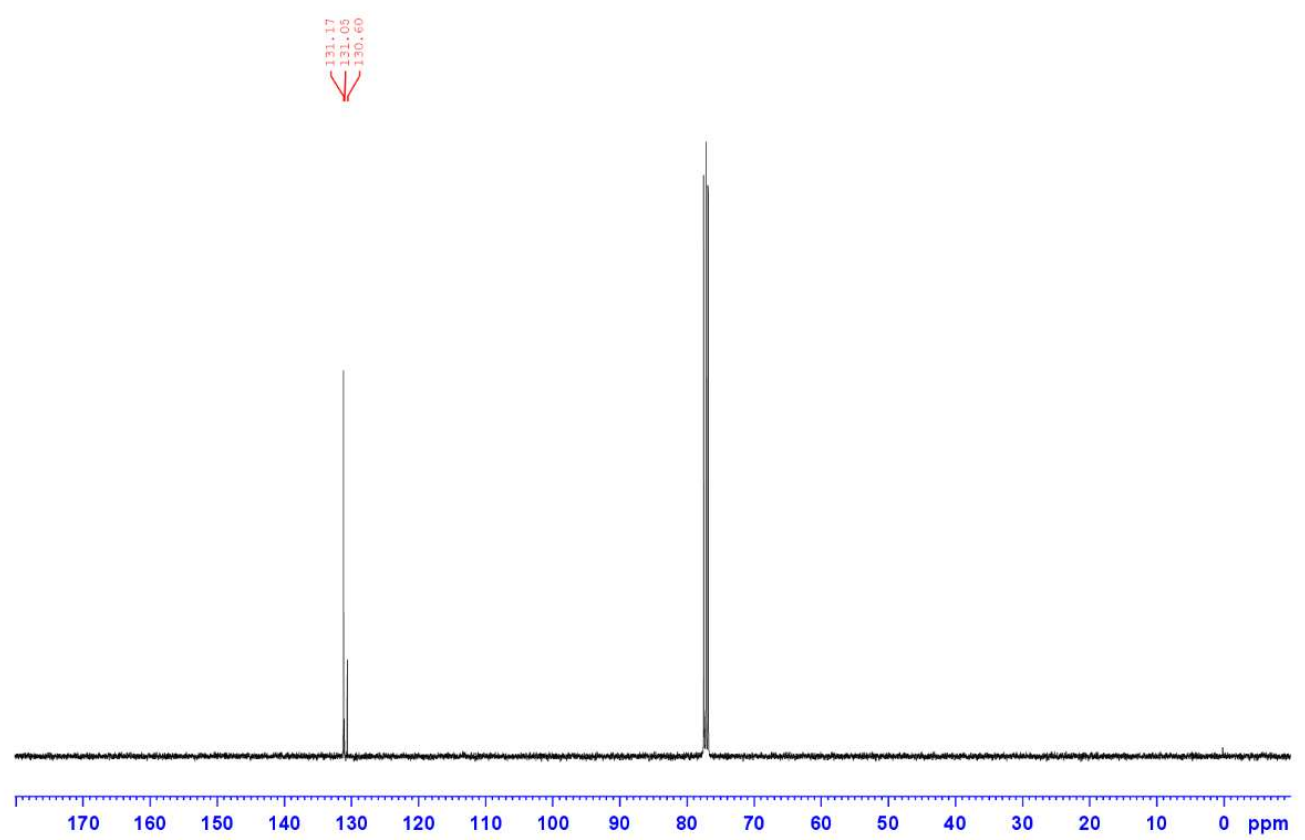


${ }^{1} \mathrm{H}$ NMR (1c-Br 2$)$

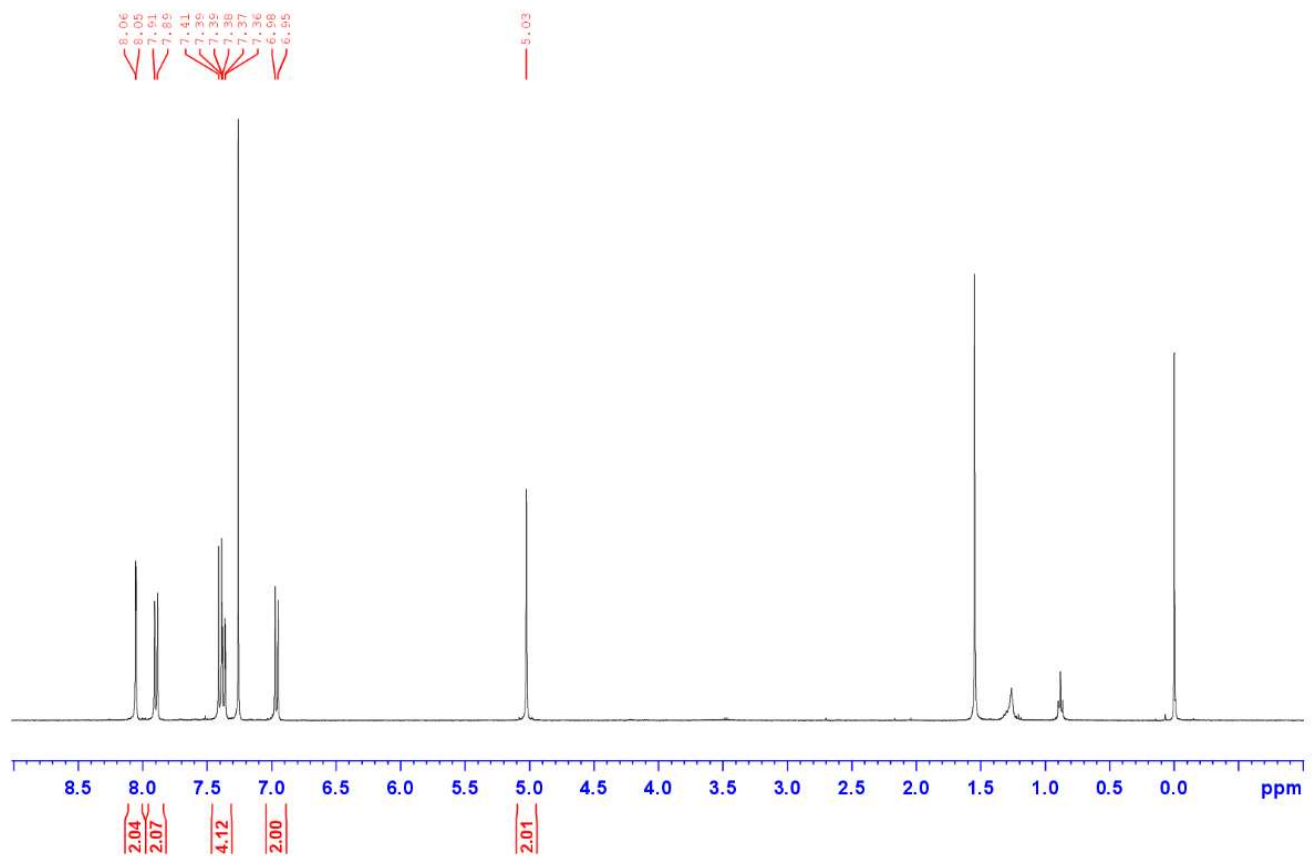

${ }^{13} \mathrm{C}$ NMR (1c-Br $)$

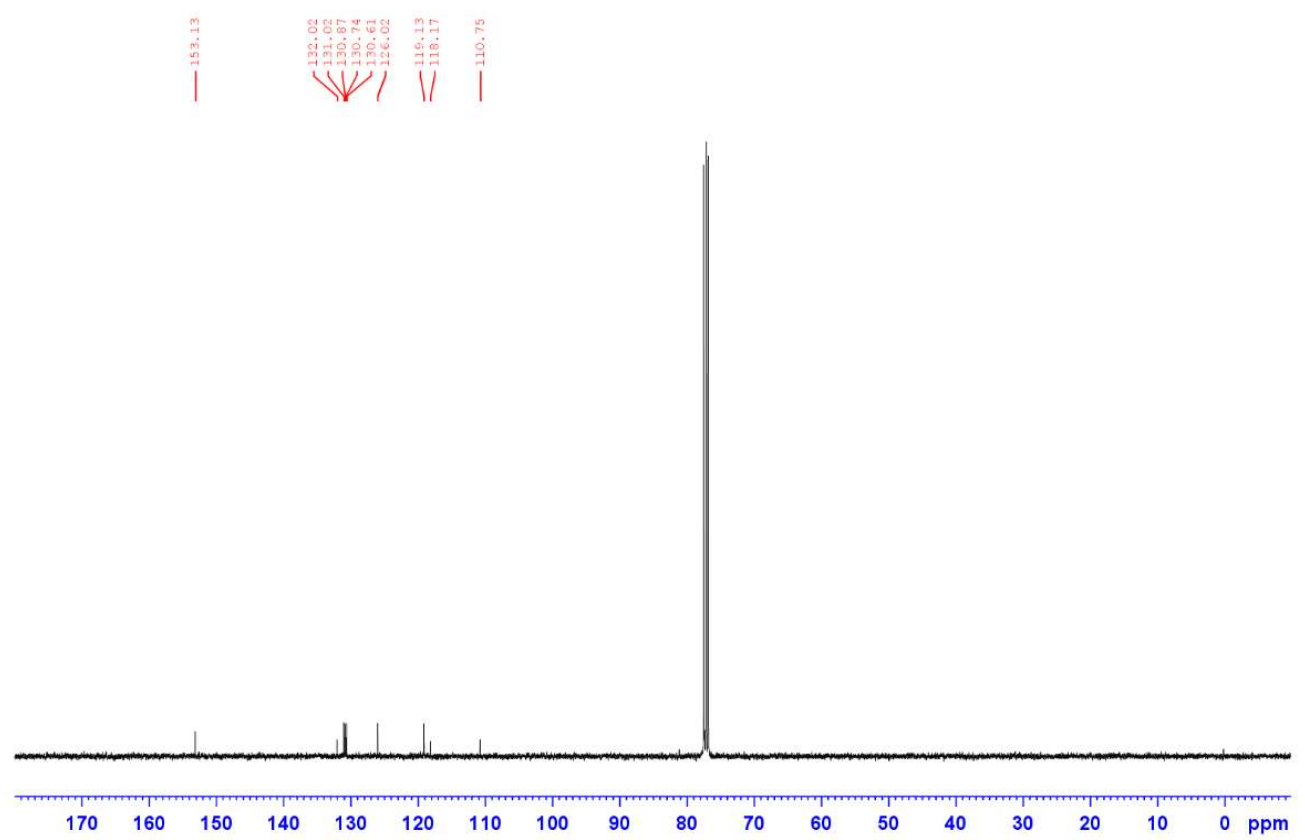


${ }^{1} \mathrm{H}$ NMR (1d-Br 2$)$

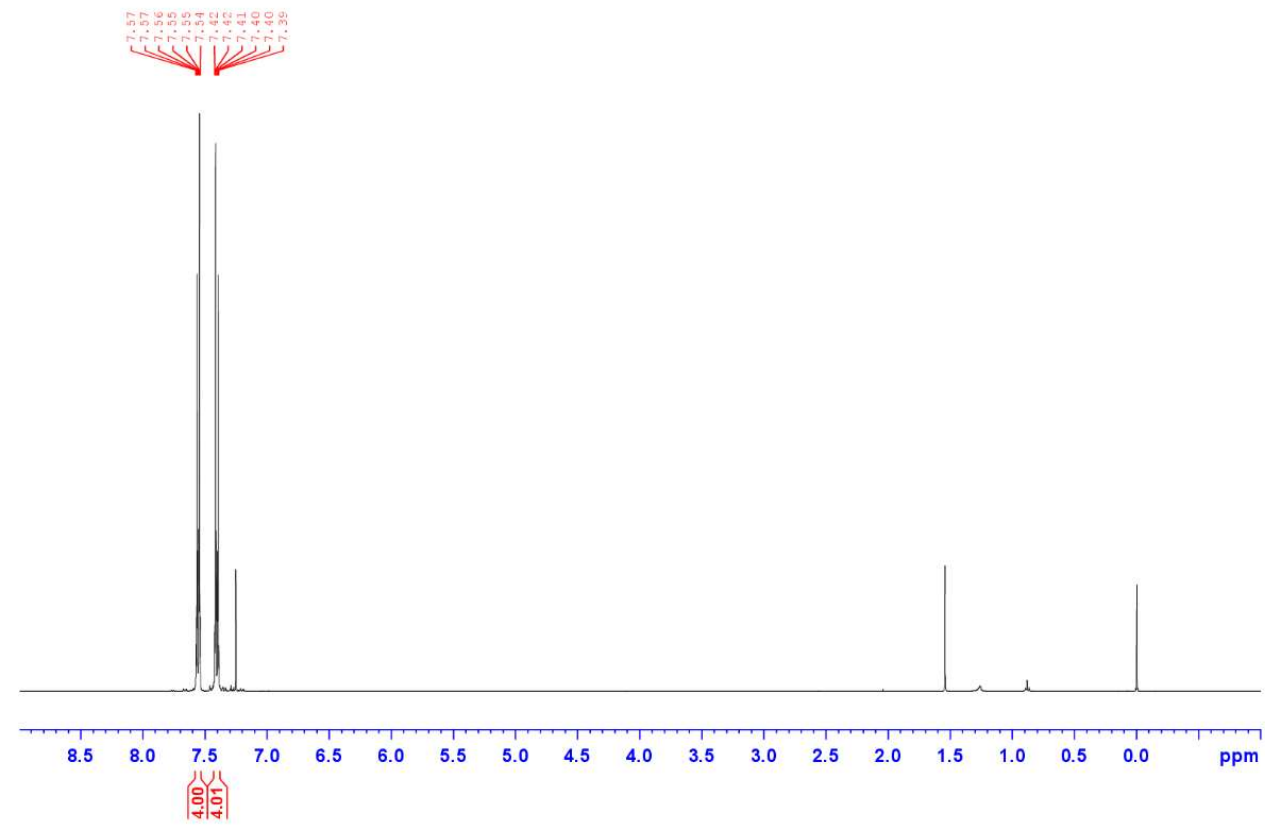

${ }^{13} \mathrm{C}$ NMR (1d-Br 2$)$

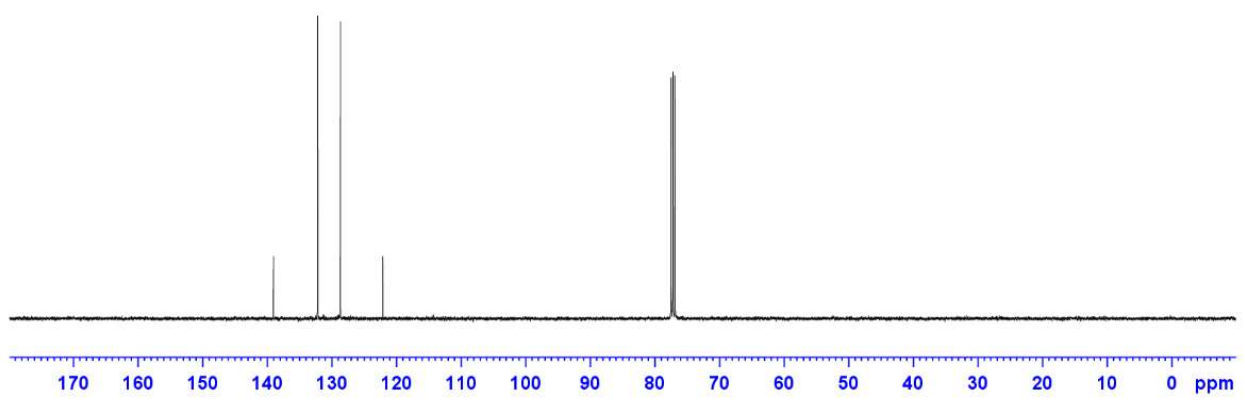


${ }^{1} \mathrm{H}$ NMR (1e-Br 2$)$

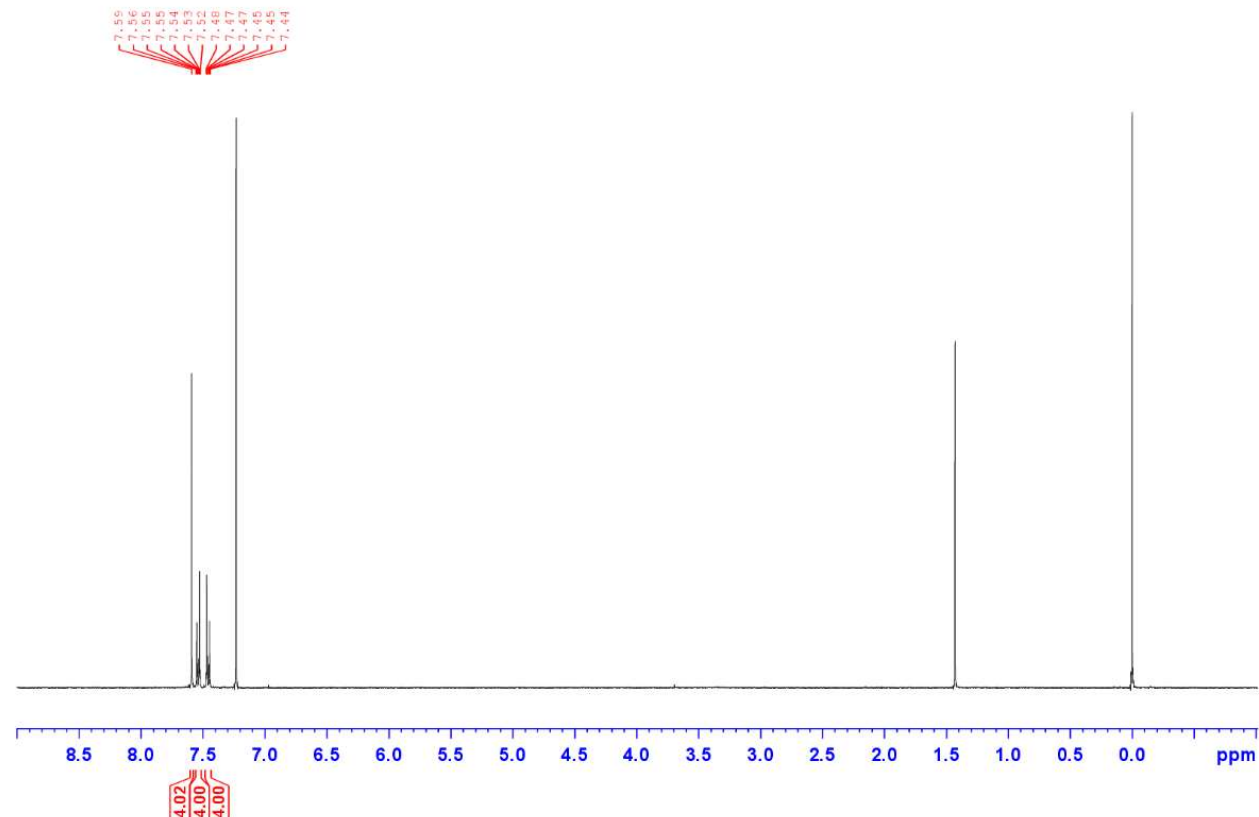

${ }^{13} \mathrm{C}$ NMR $\left(\mathbf{1 e}-\mathrm{Br}_{2}\right)$

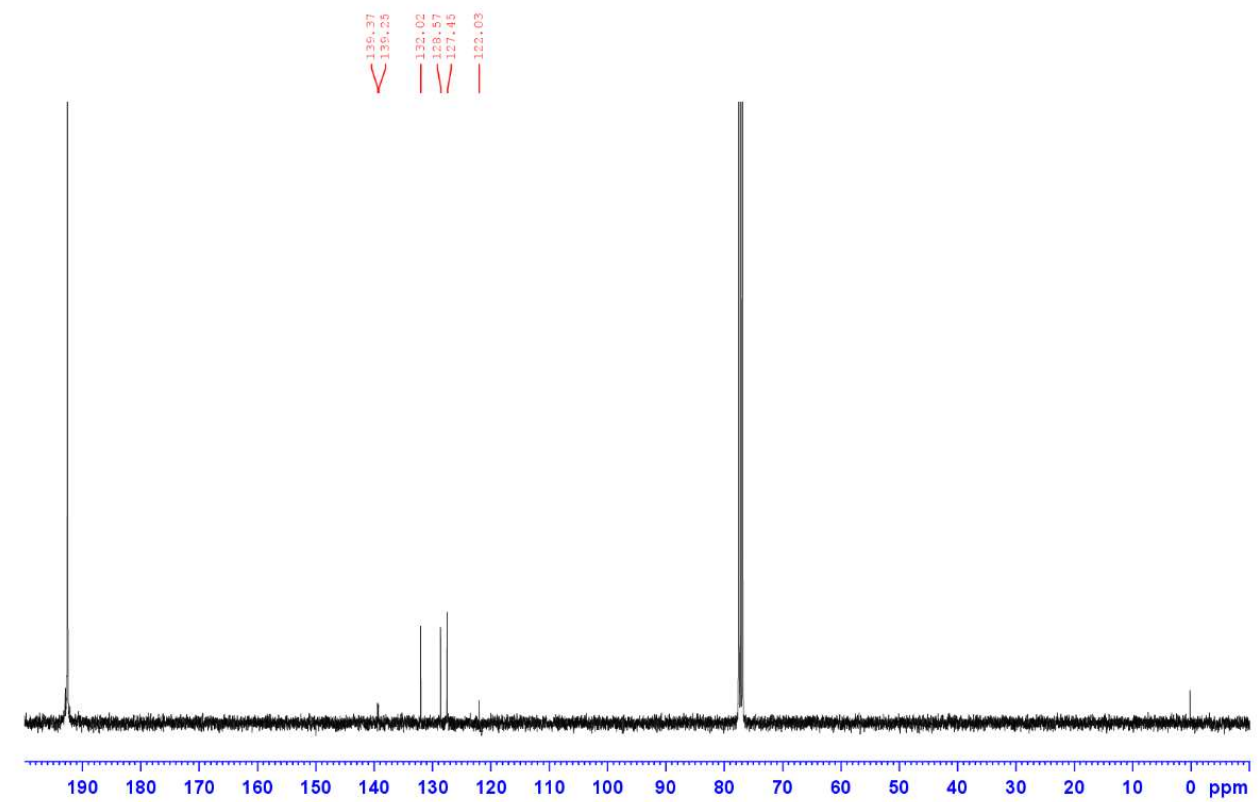


${ }^{1} \mathrm{H}$ NMR (1f- $\left.-\mathrm{Br}_{2}\right)$

$\mathrm{V} v \mathrm{~V}$

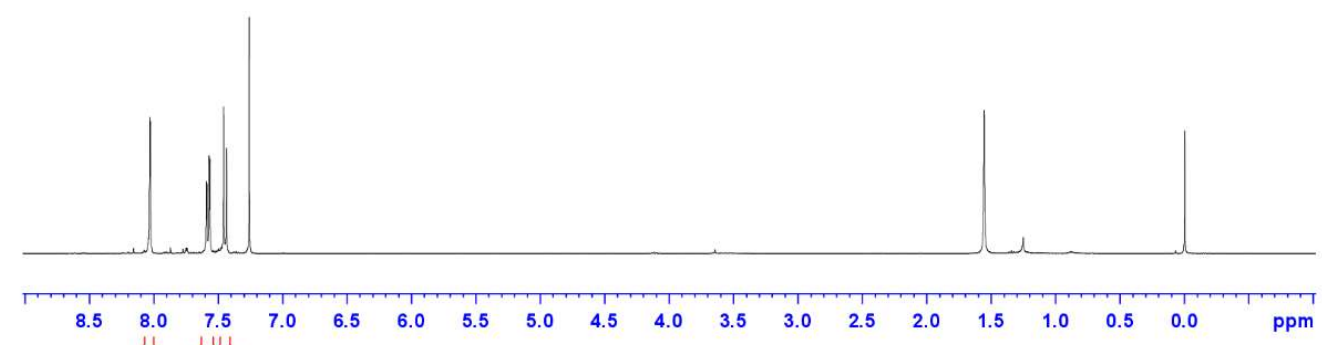

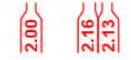

${ }^{13} \mathrm{C}$ NMR (1f $\left.-\mathrm{Br}_{2}\right)$

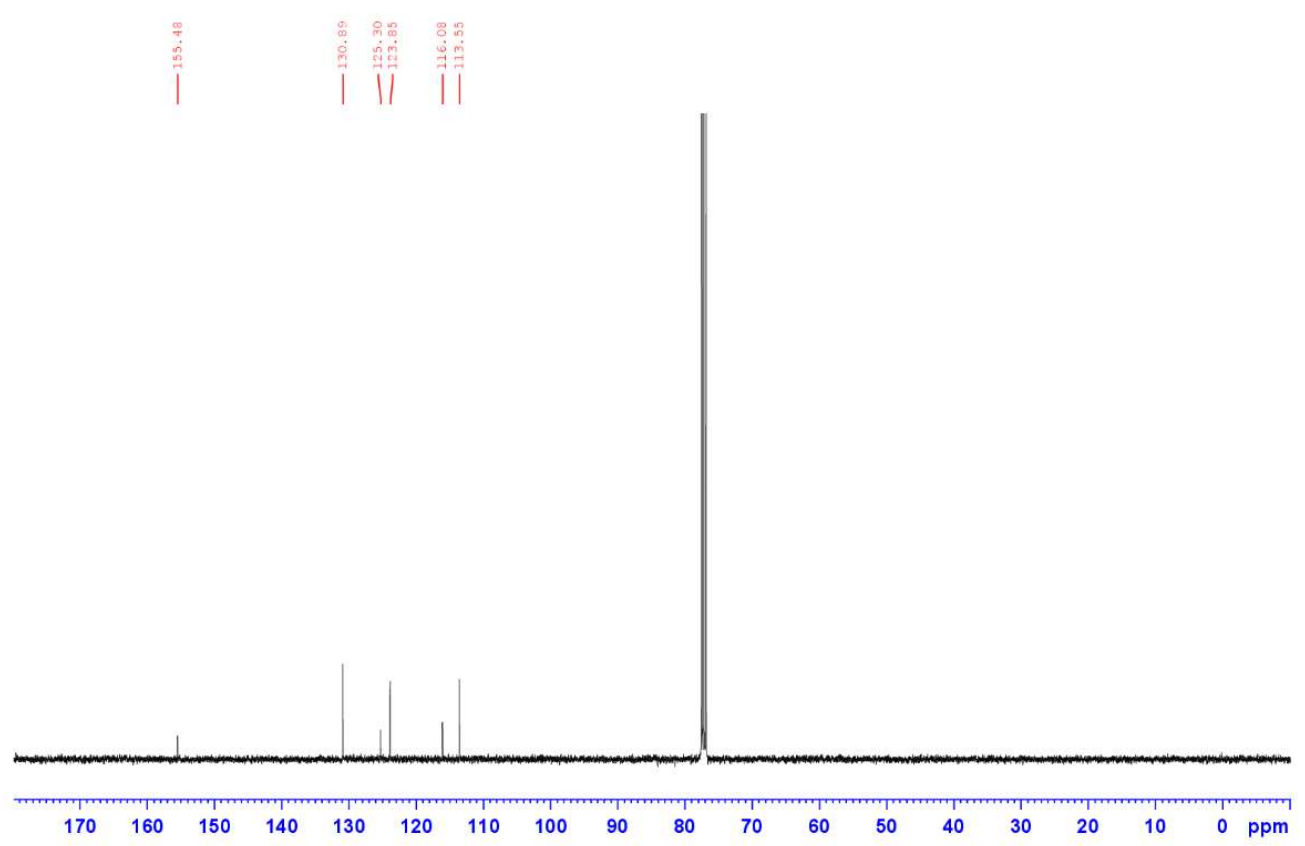


${ }^{1} \mathrm{H}$ NMR (1g-Br 2$)$

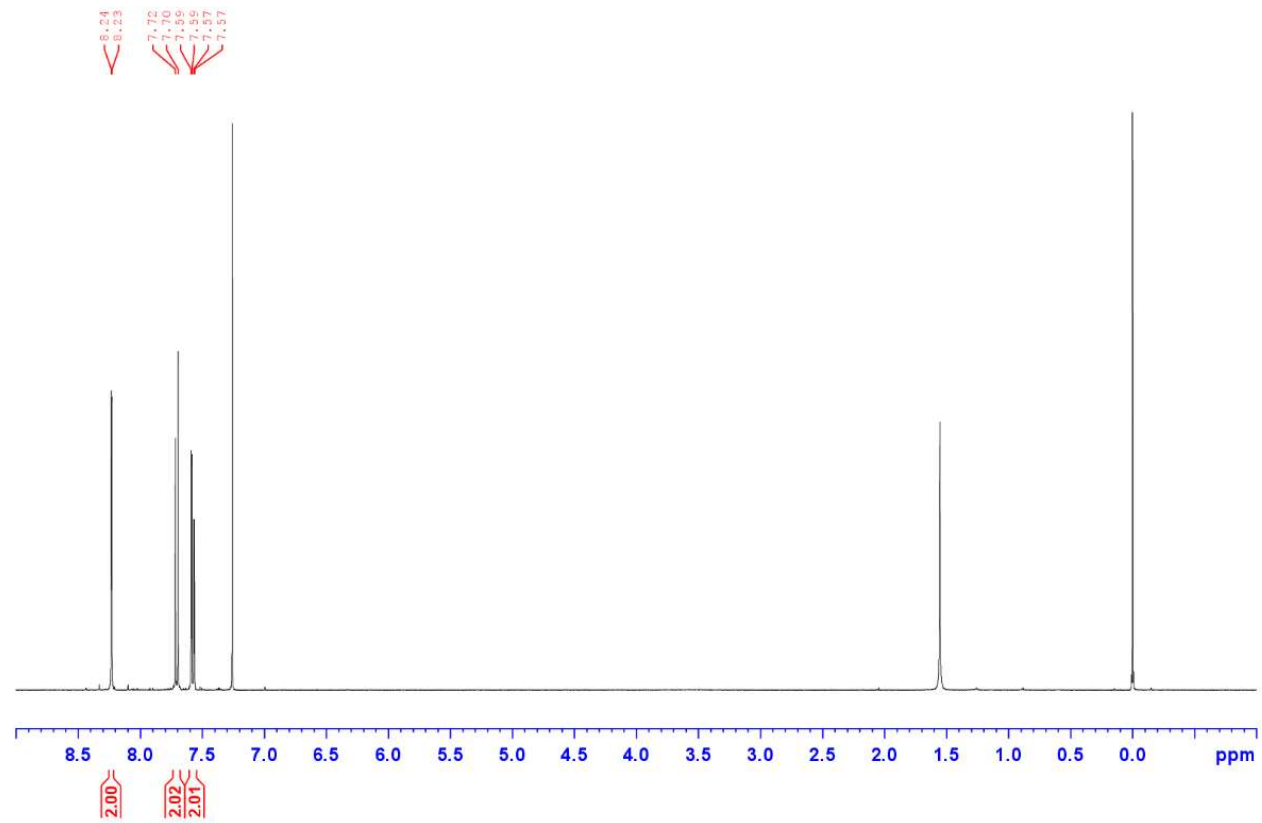

${ }^{13} \mathrm{C}$ NMR $\left(\mathbf{1 g}-\mathrm{Br}_{2}\right)$

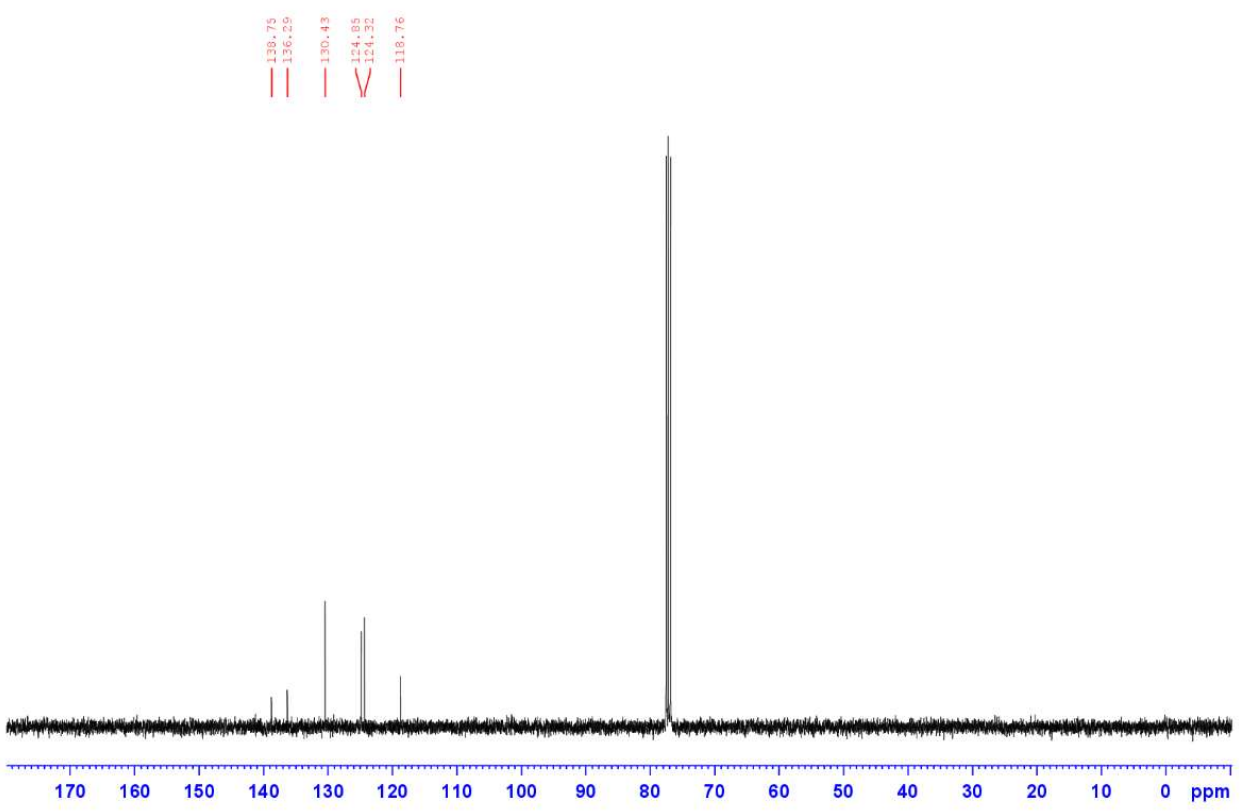


${ }^{1} \mathrm{H}$ NMR (1h-Br $\left.{ }_{4}\right)$

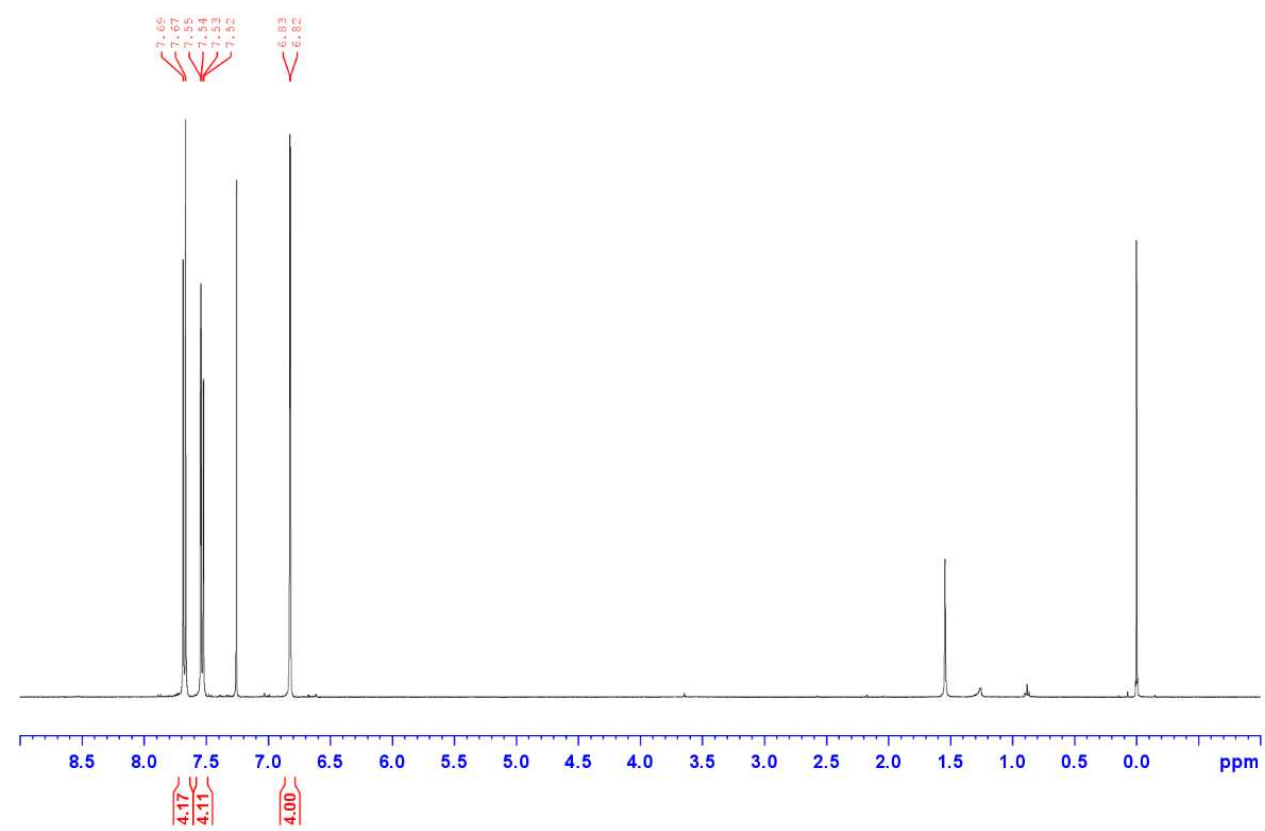

${ }^{13} \mathrm{C}$ NMR (1h-Br 4$)$

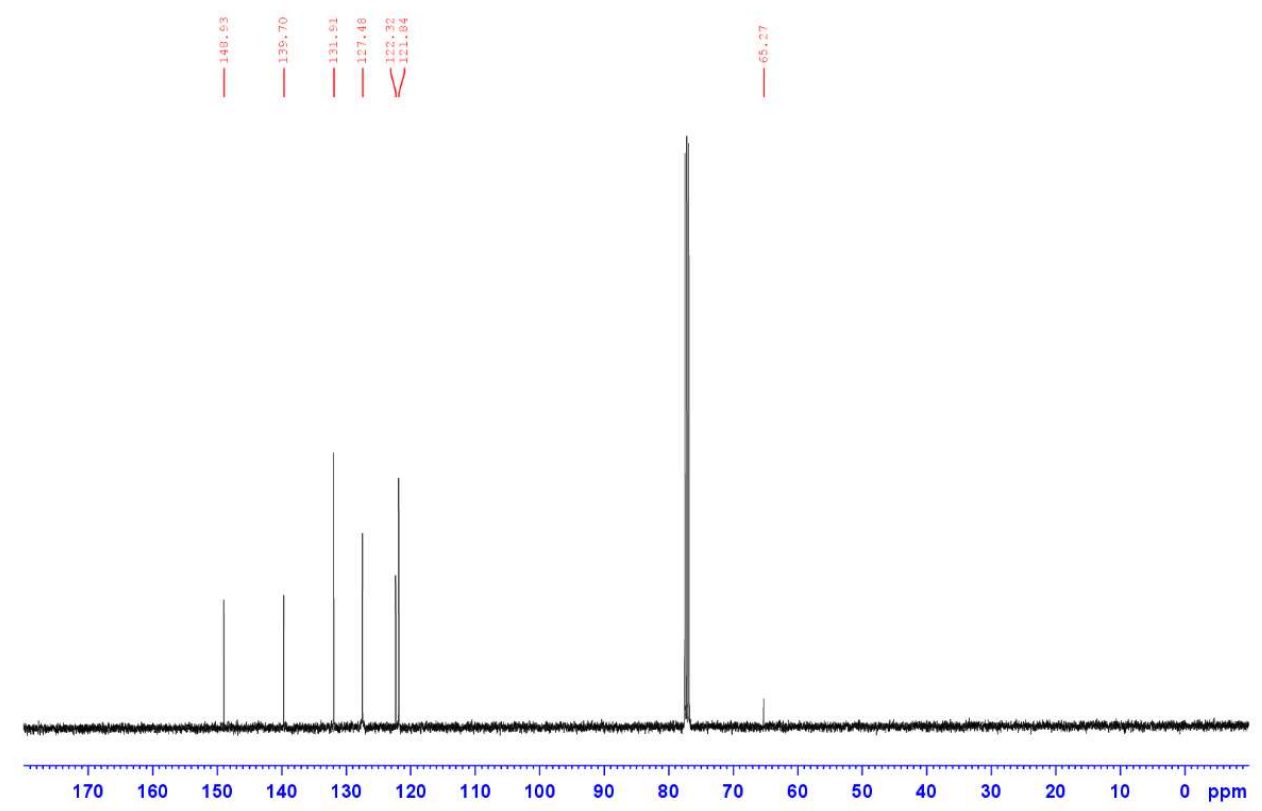


${ }^{1} \mathrm{H}$ NMR (1i-para-Br 4$)$

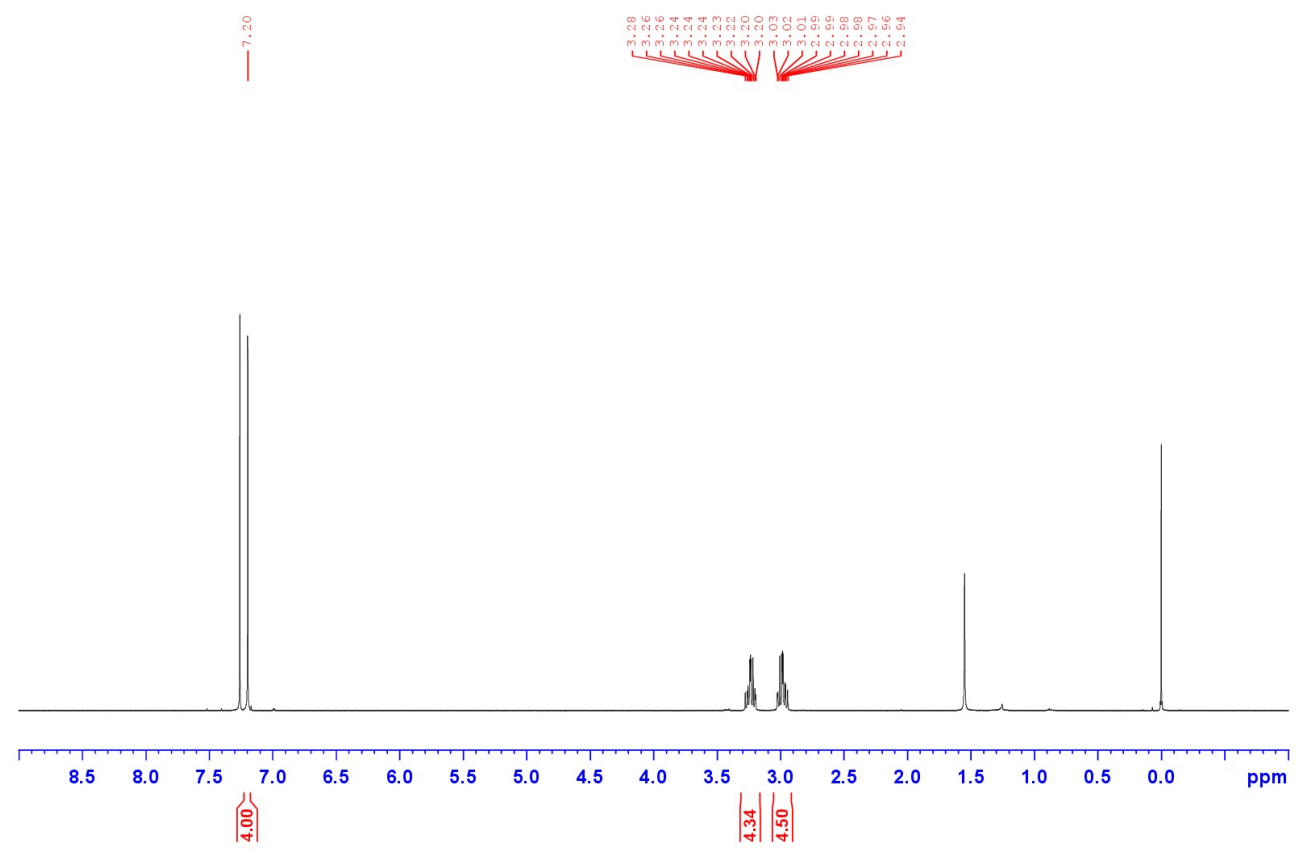

${ }^{13}$ C NMR (1i-para-Br 4 )

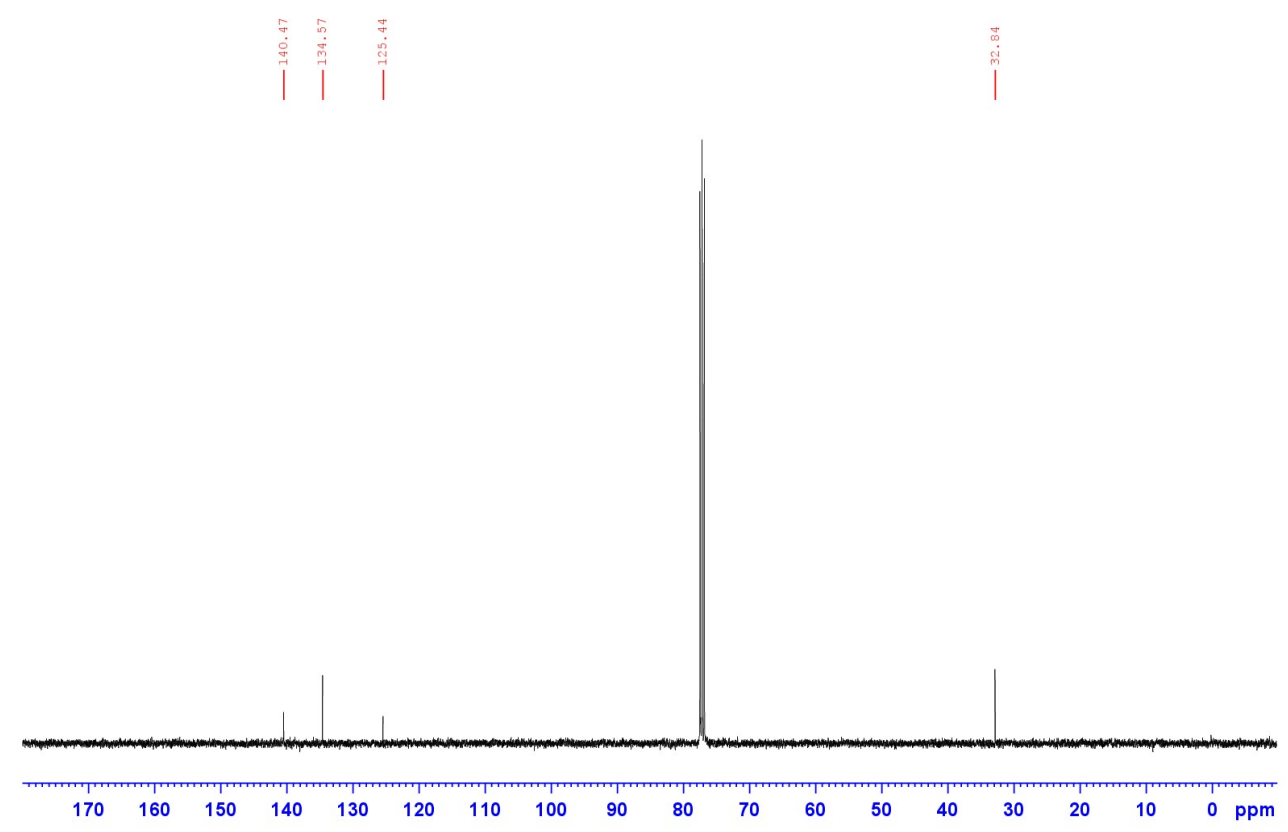


${ }^{1} \mathrm{H}$ NMR (1i-ortho-Br4)

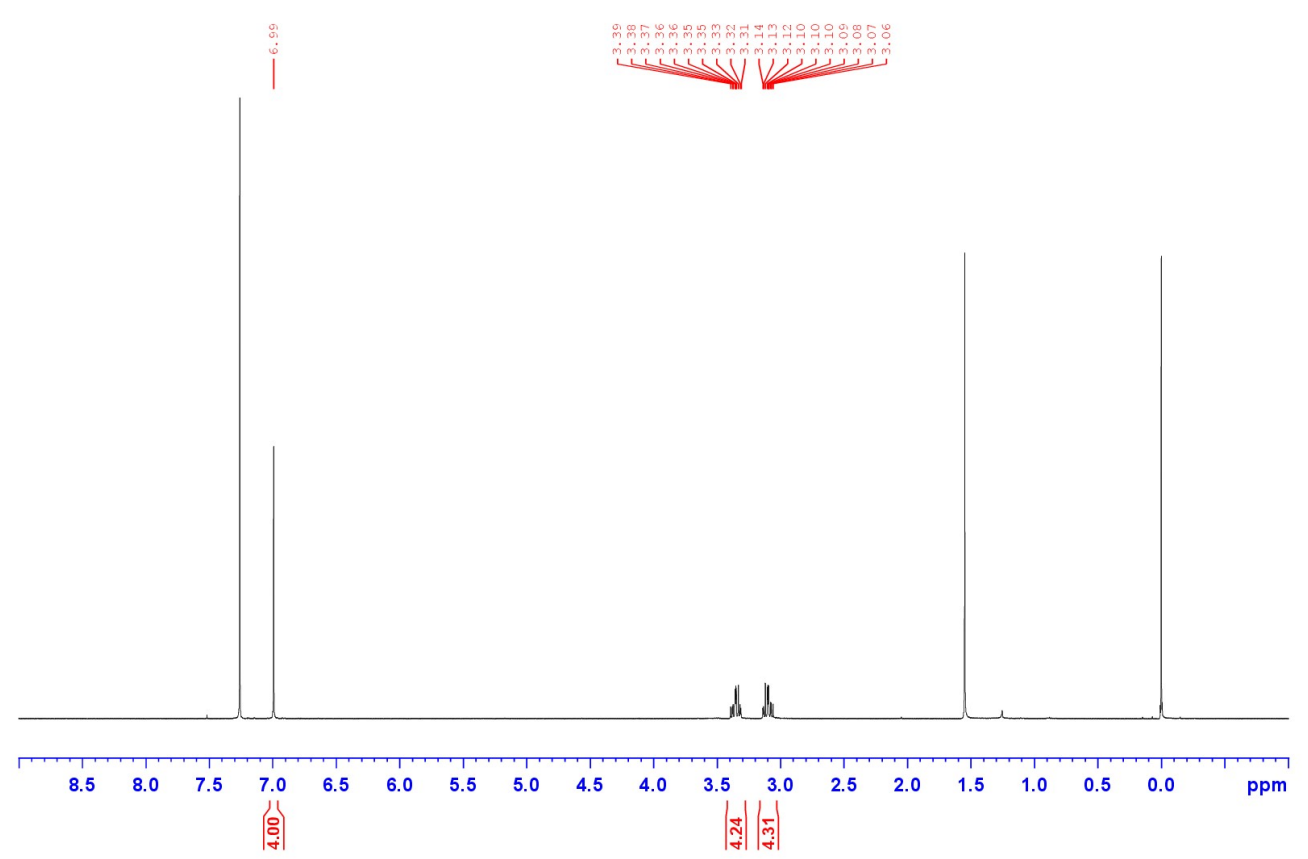

${ }^{13} \mathrm{C}$ NMR (1i-ortho-Br 4 )

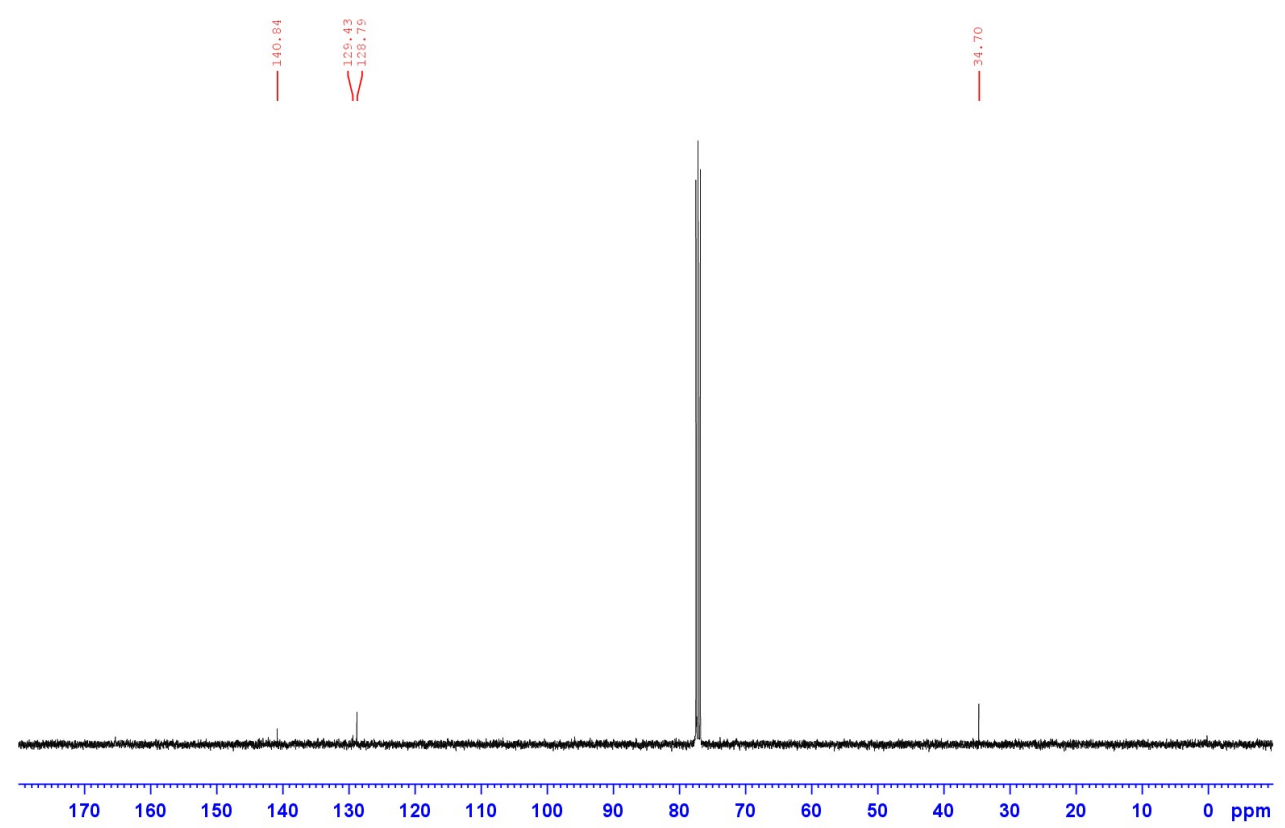


${ }^{1} \mathrm{H}$ NMR (1j-Cl (C1))
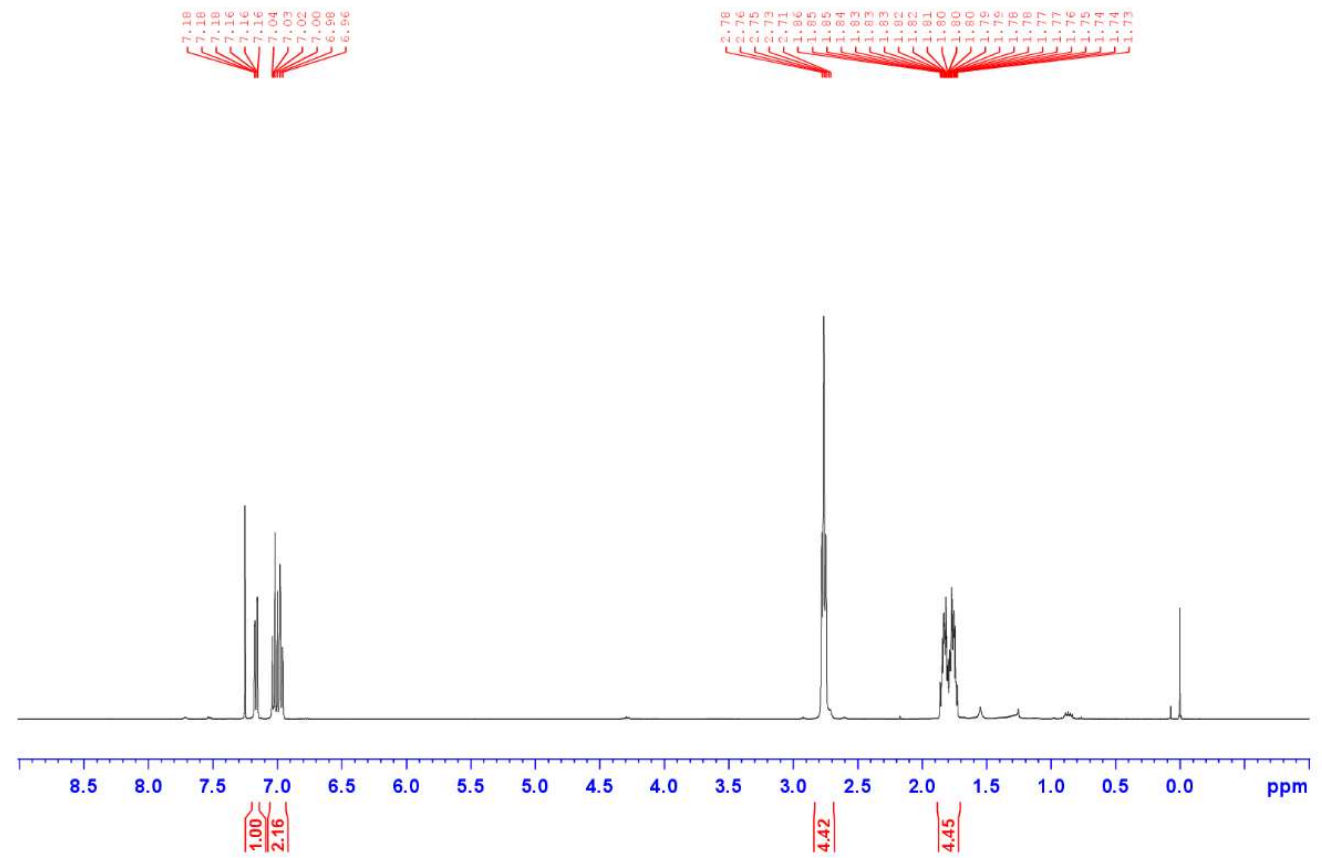

${ }^{13} \mathrm{C}$ NMR (1j-Cl (C1))

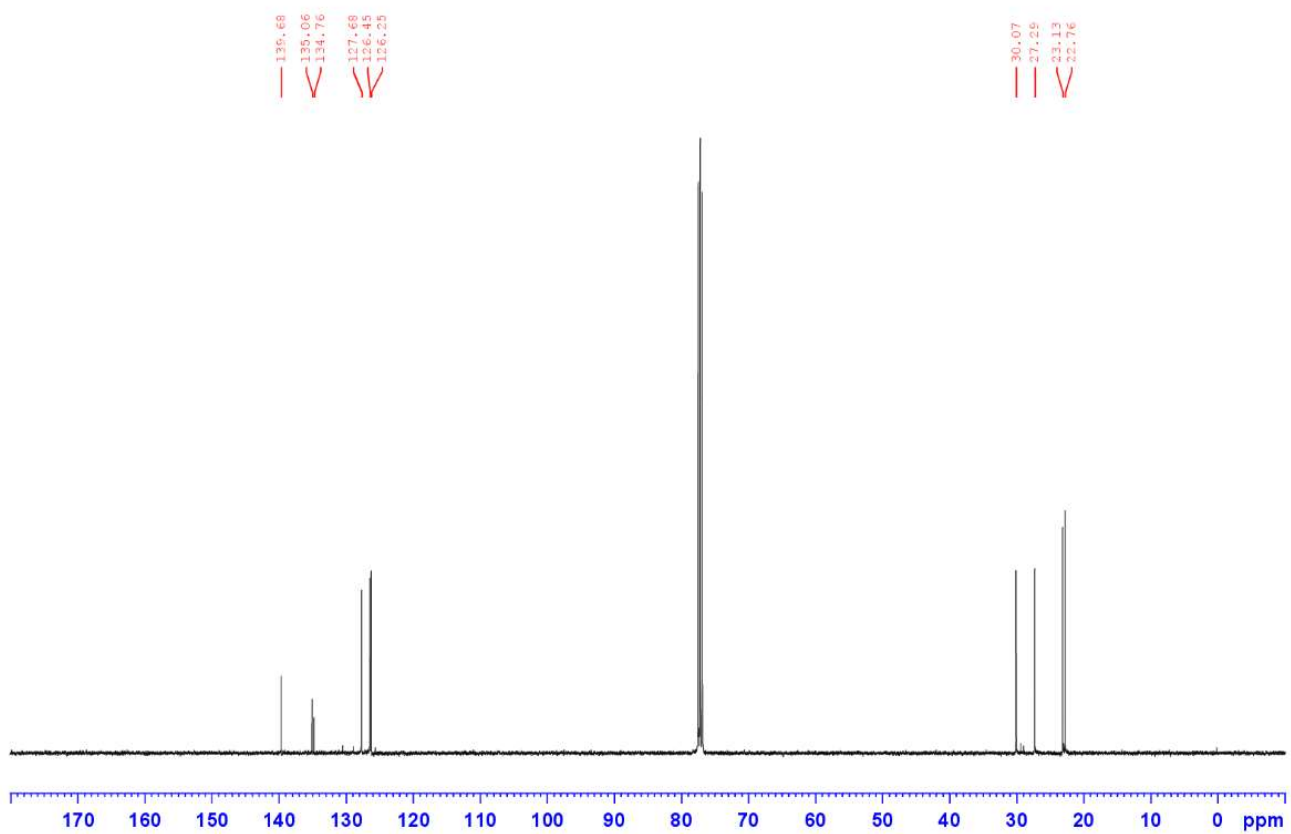


${ }^{1} \mathrm{H} \operatorname{NMR}(\mathbf{1 j}-\mathrm{Cl}(\mathrm{C} 2))$

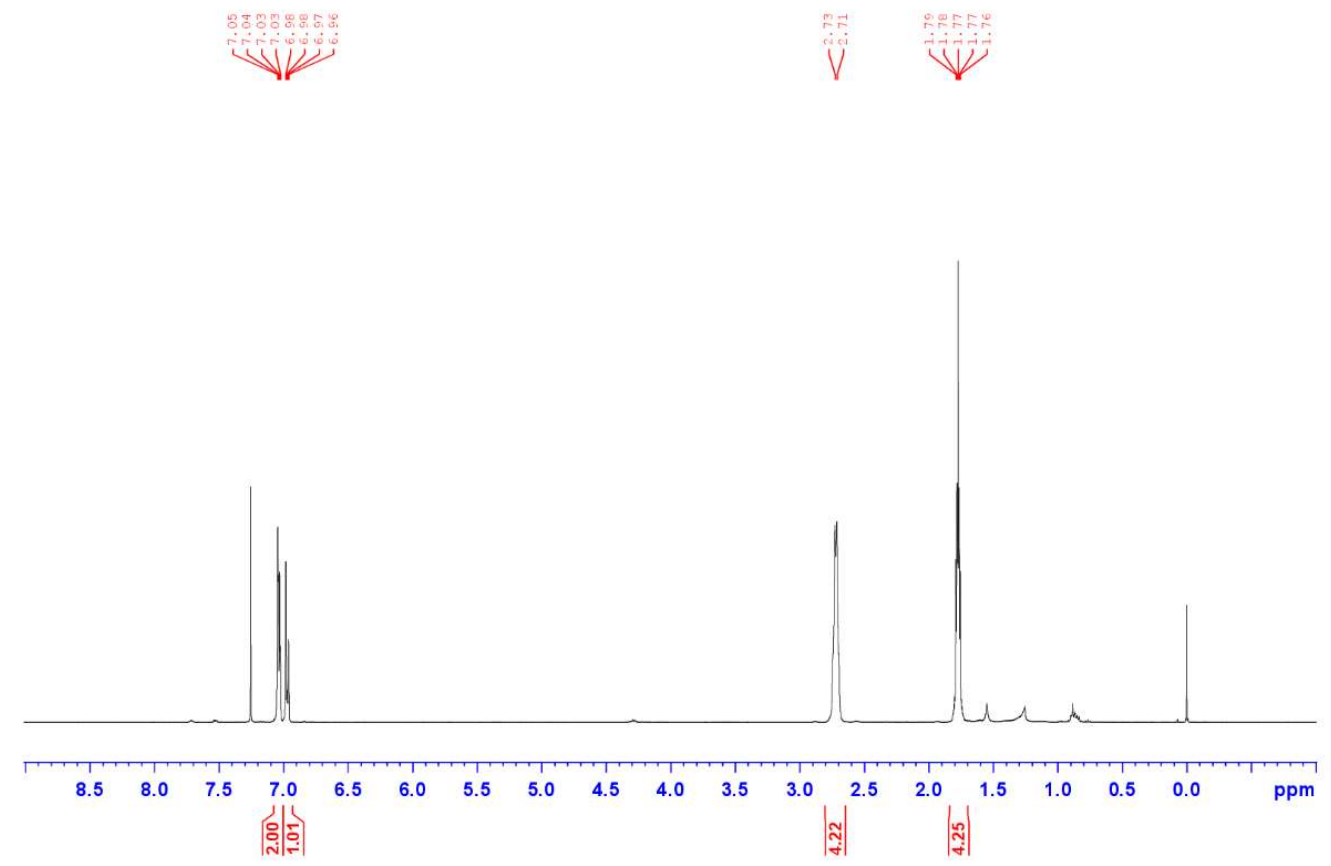

${ }^{13} \mathrm{C}$ NMR (1j-Cl (C2))

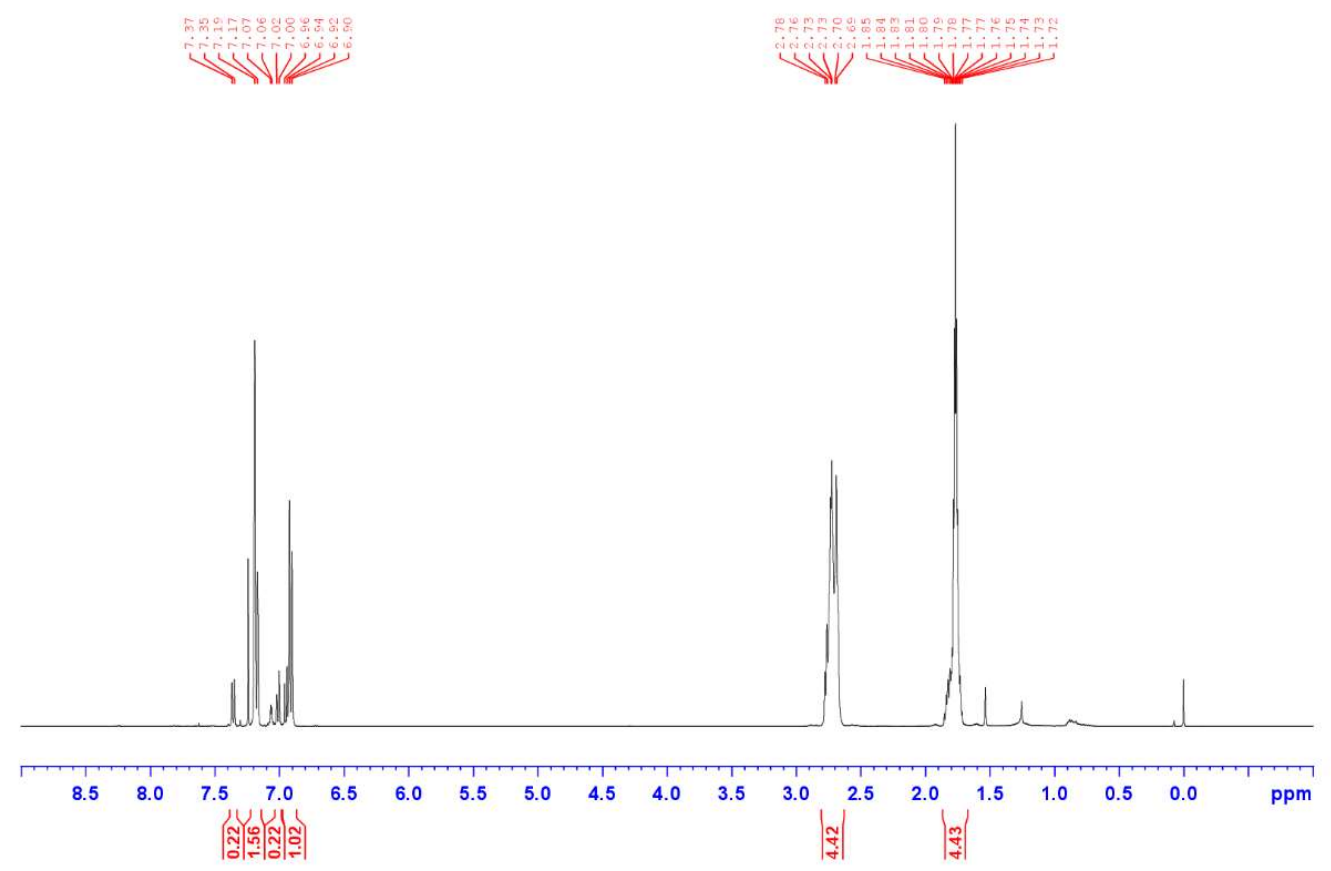


${ }^{1} \mathrm{H}$ NMR (1j-Br)

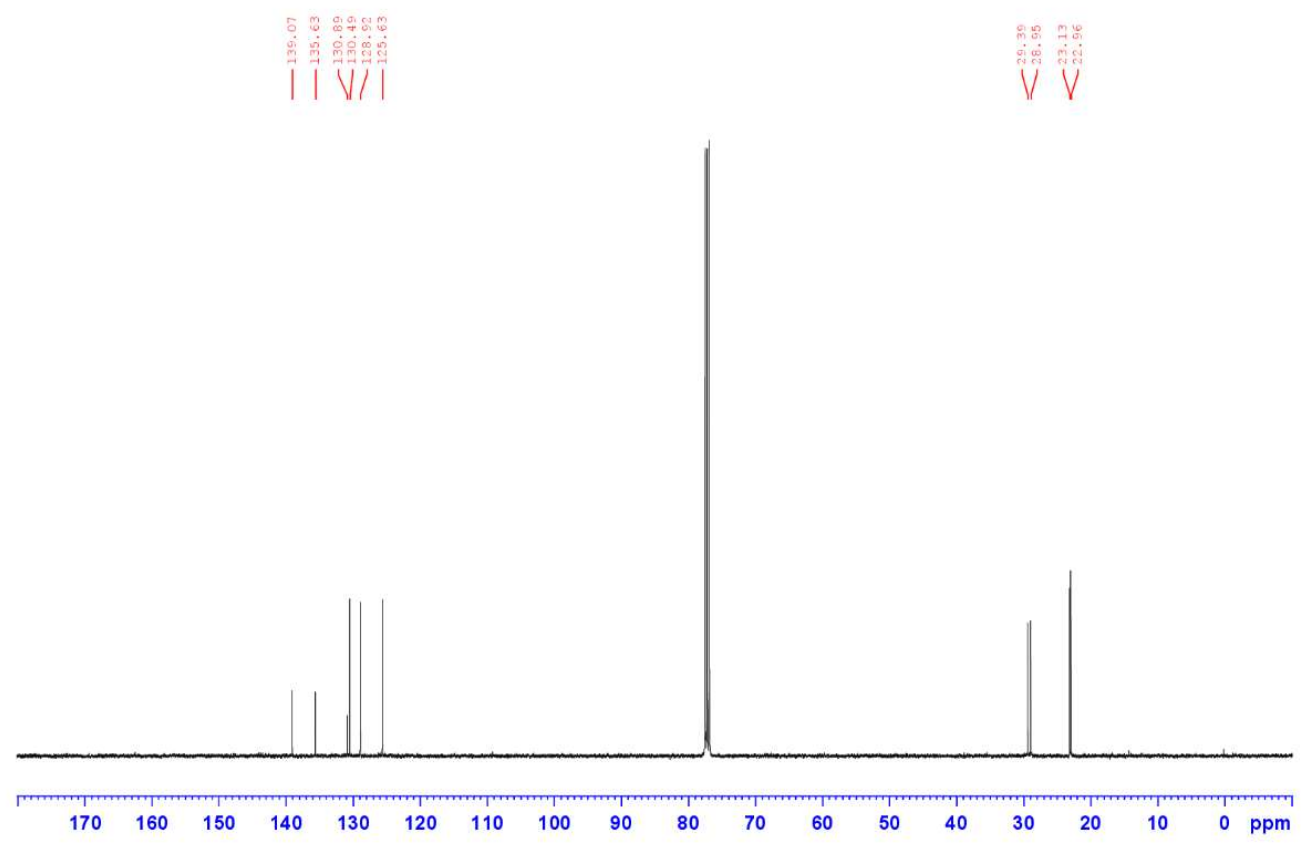

${ }^{13} \mathrm{C}$ NMR $(\mathbf{1} \mathbf{j}-\mathrm{Br})$

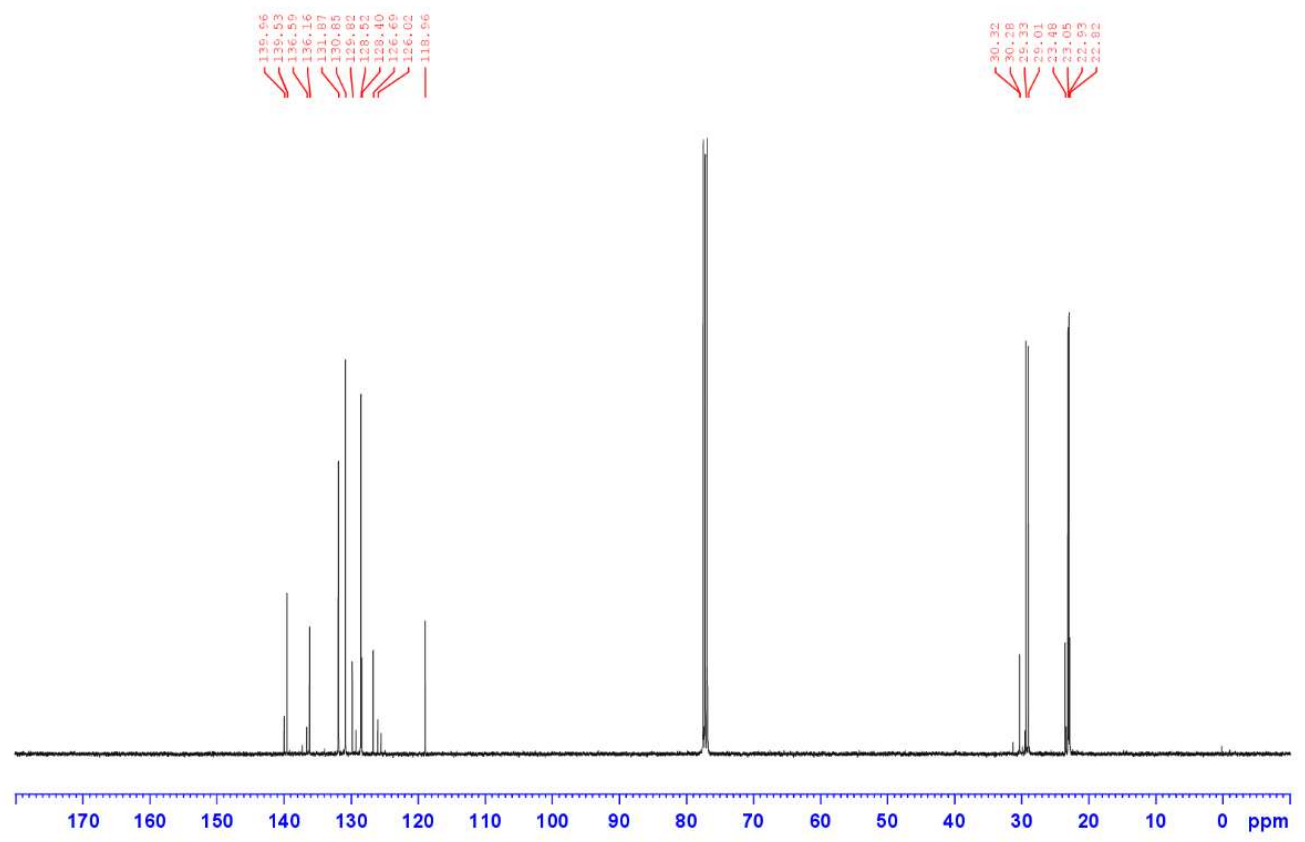


${ }^{1} \mathrm{H}$ NMR (1j-I)

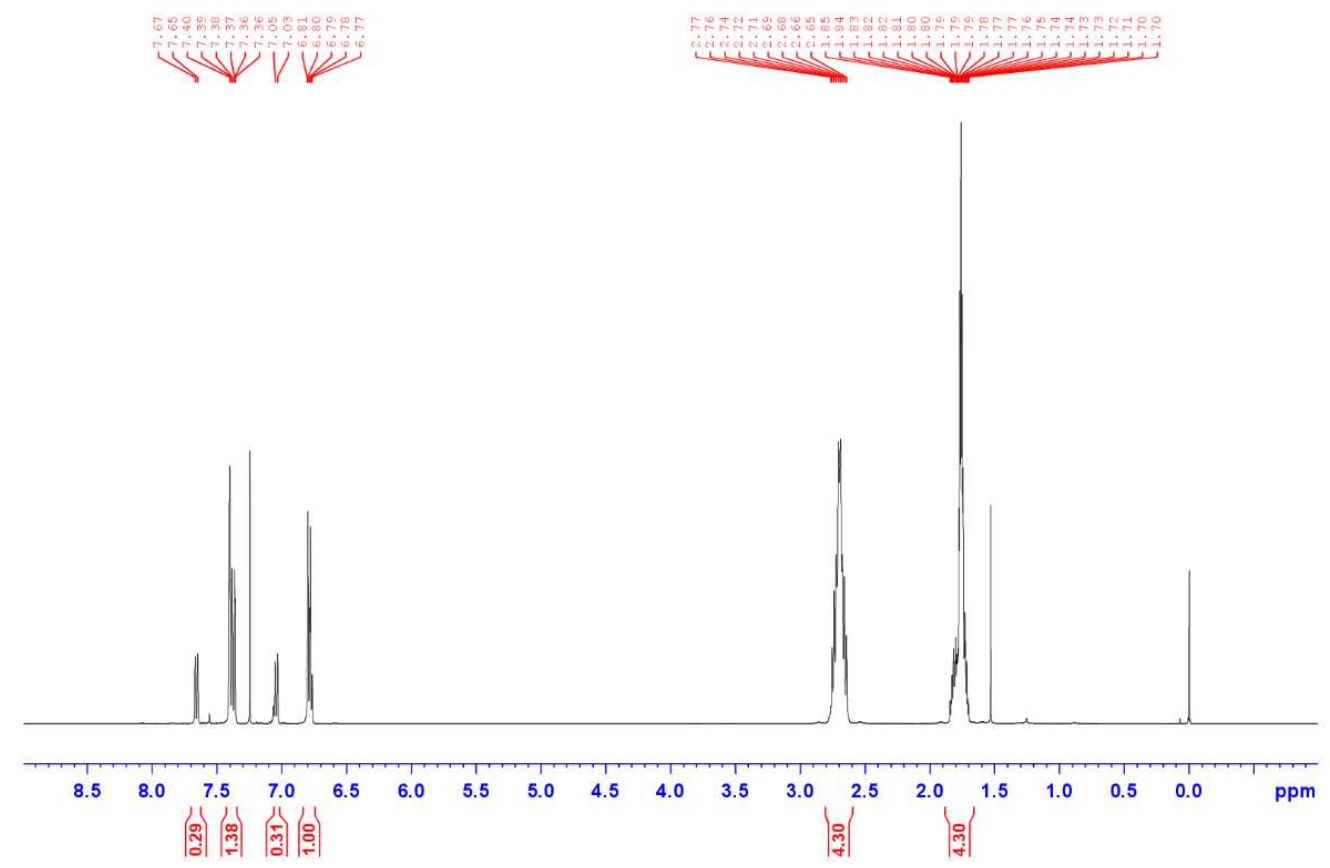

${ }^{13} \mathrm{C}$ NMR (1j-I)

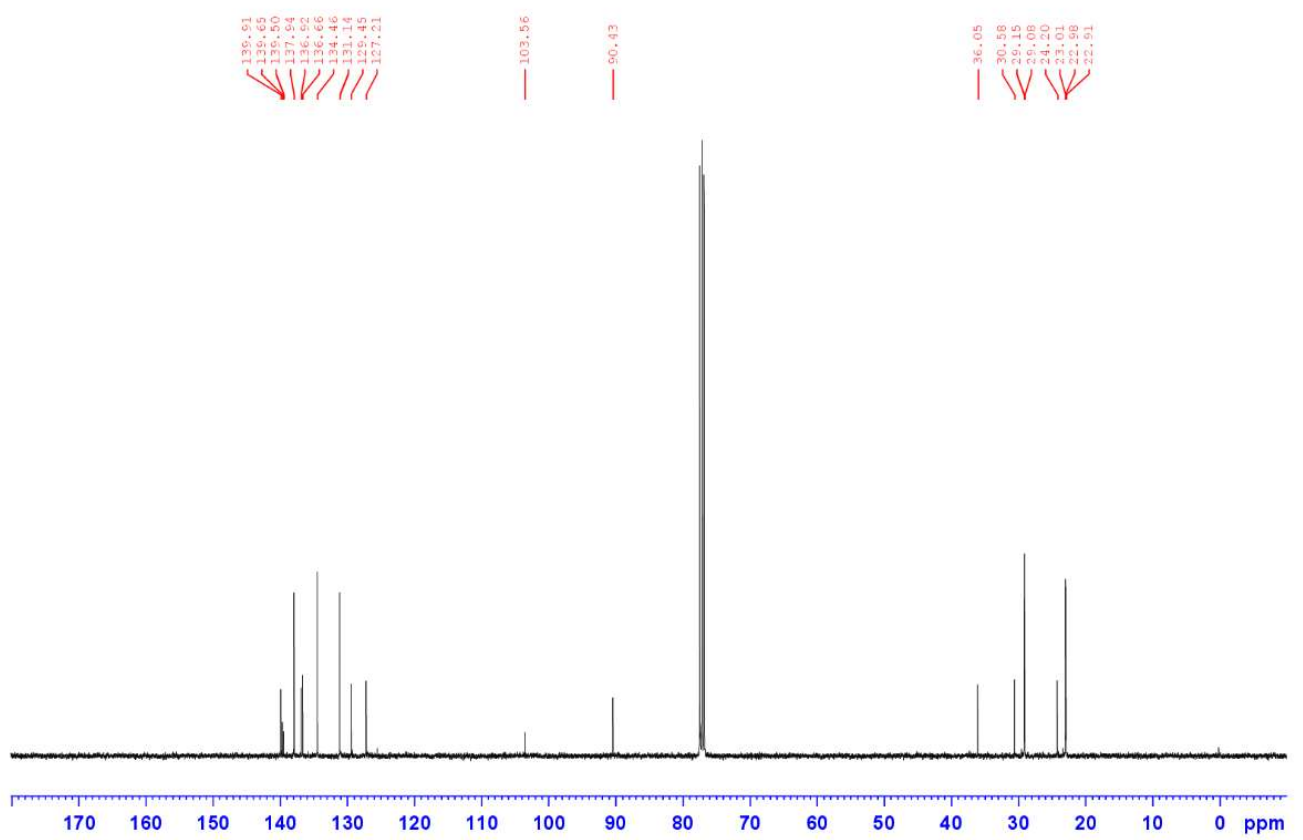


${ }^{1} \mathrm{H}$ NMR (1 m-Cl)

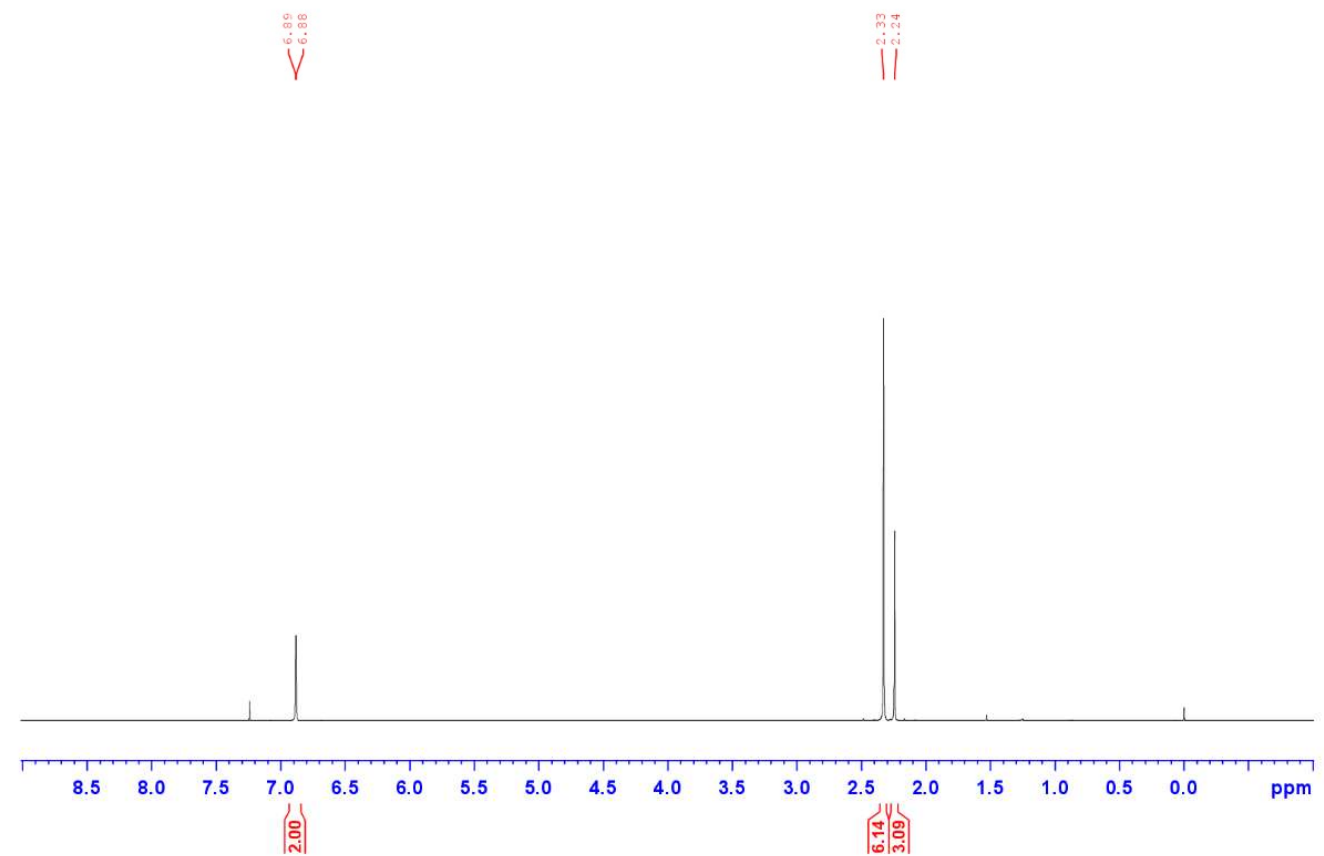

${ }^{13} \mathrm{C}$ NMR (1m-Cl)

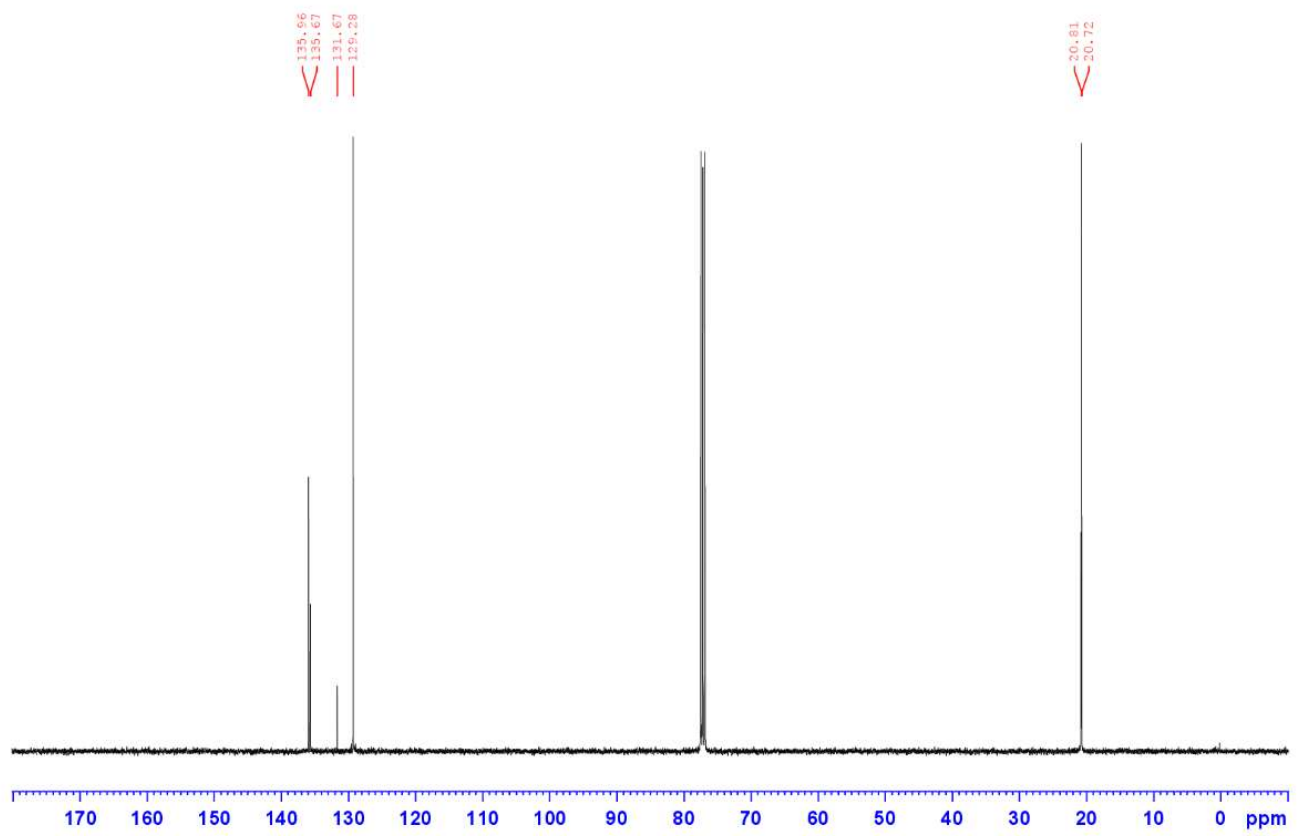


${ }^{1} \mathrm{H}$ NMR (1 m-Br)

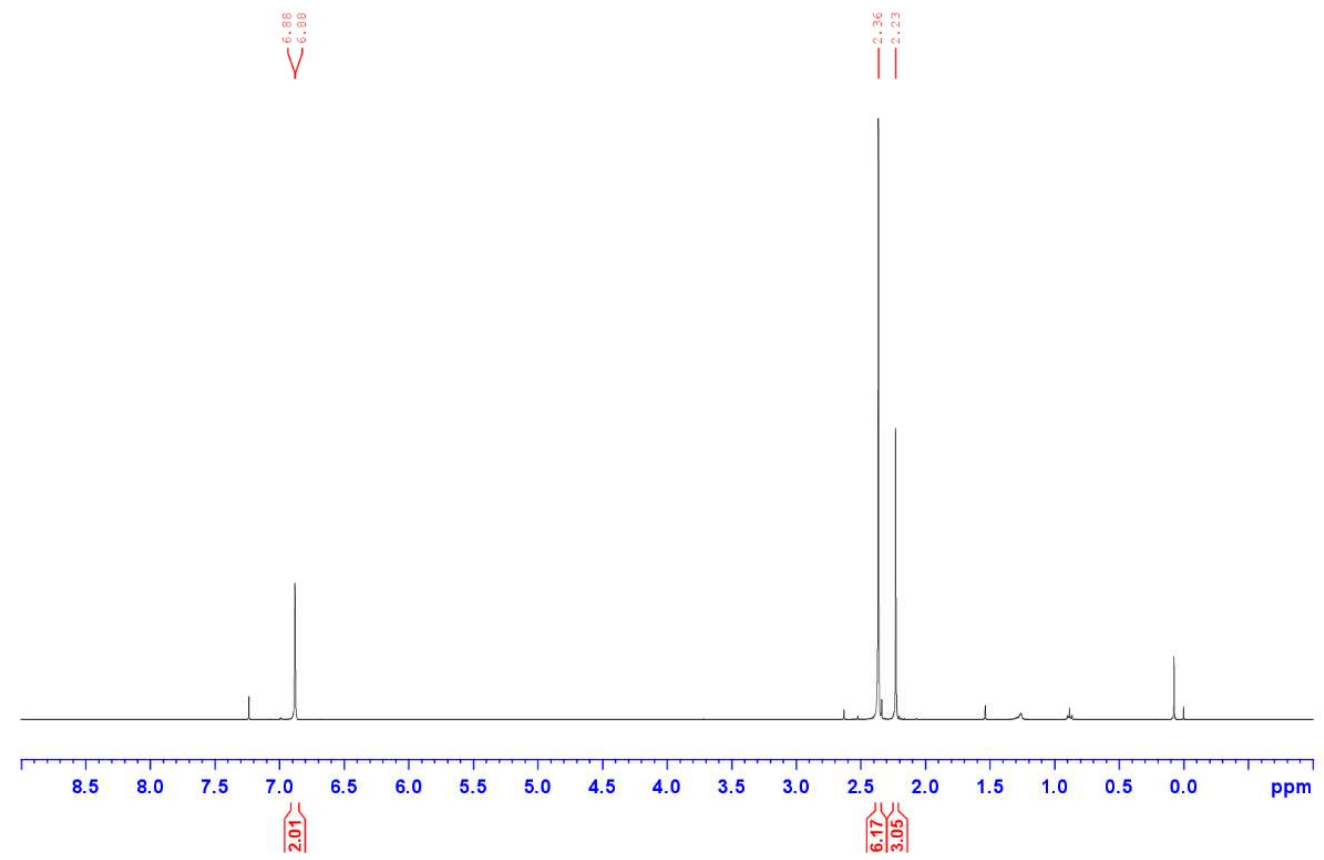

${ }^{13} \mathrm{C}$ NMR (1m-Br)
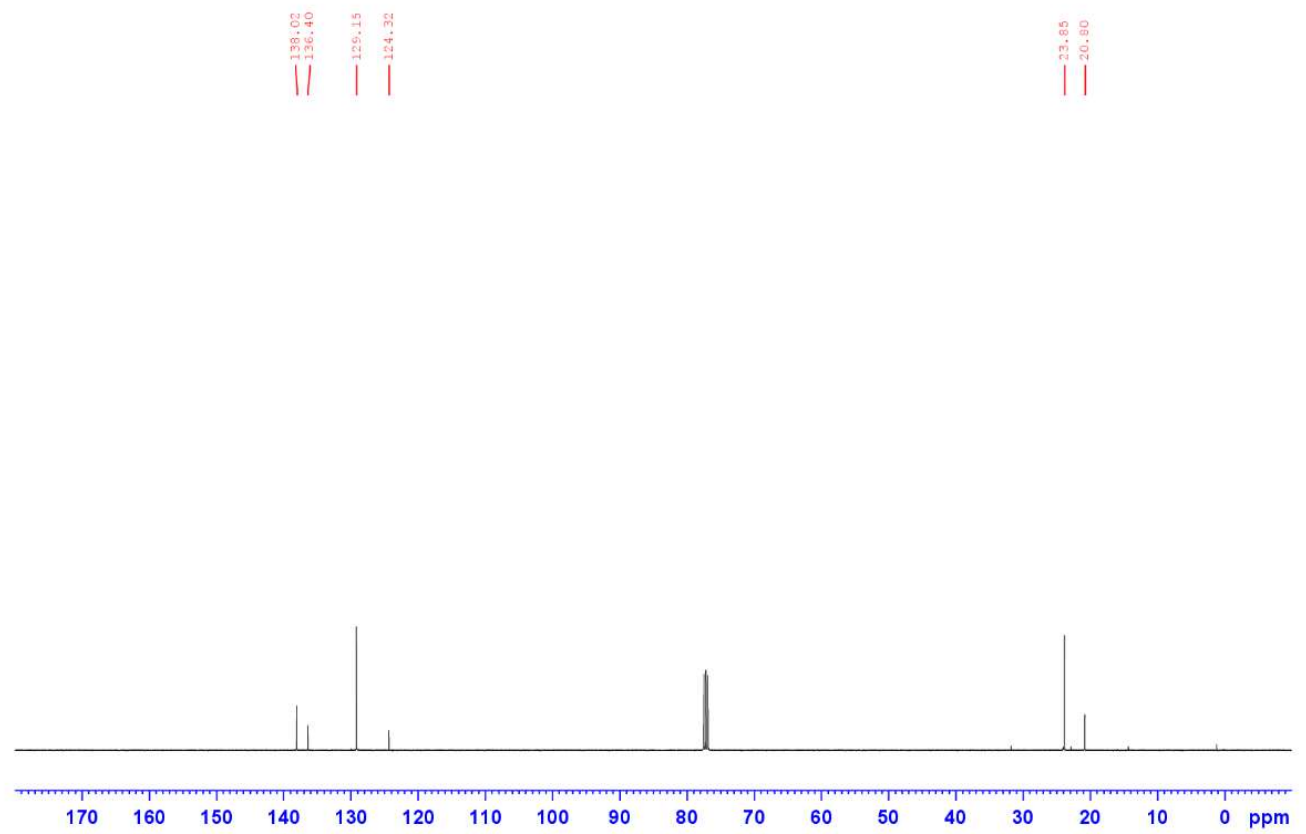
${ }^{1} \mathrm{H}$ NMR $\left(\mathbf{1 n}-\mathrm{Cl}_{2}\right)$

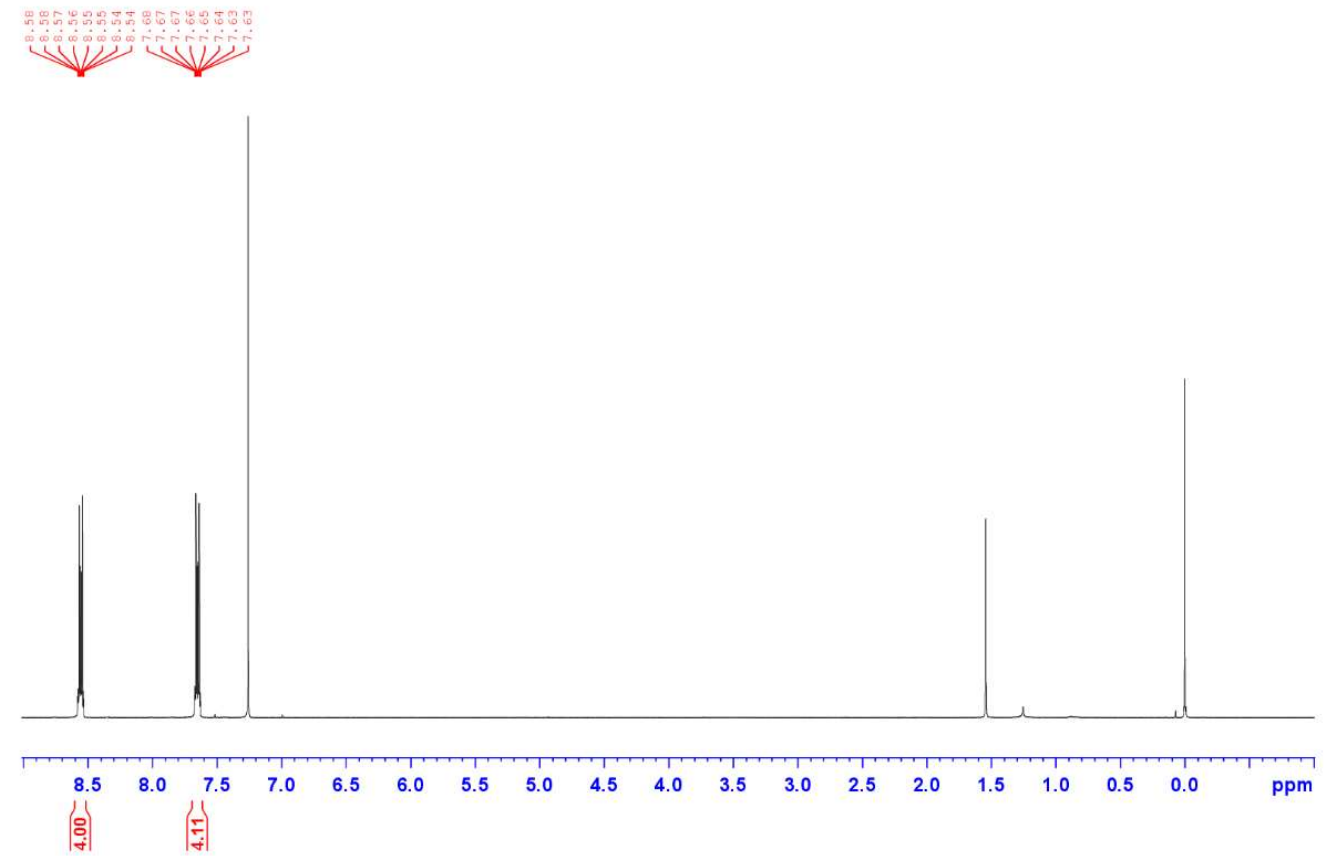

${ }^{13} \mathrm{C}$ NMR $\left(\mathbf{1 n}-\mathrm{Cl}_{2}\right)$

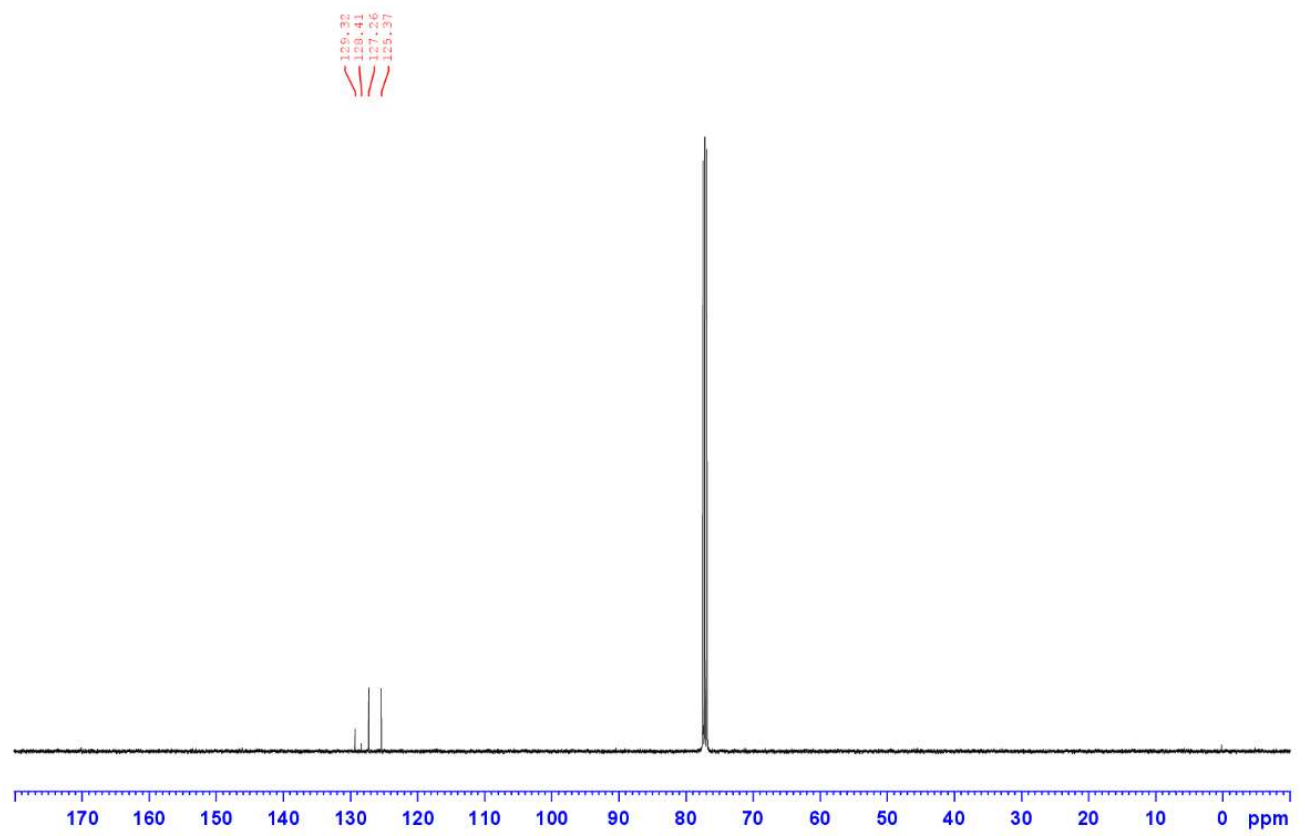


${ }^{1} \mathrm{H}$ NMR (1n- $\left.-\mathrm{Br}_{2}\right)$

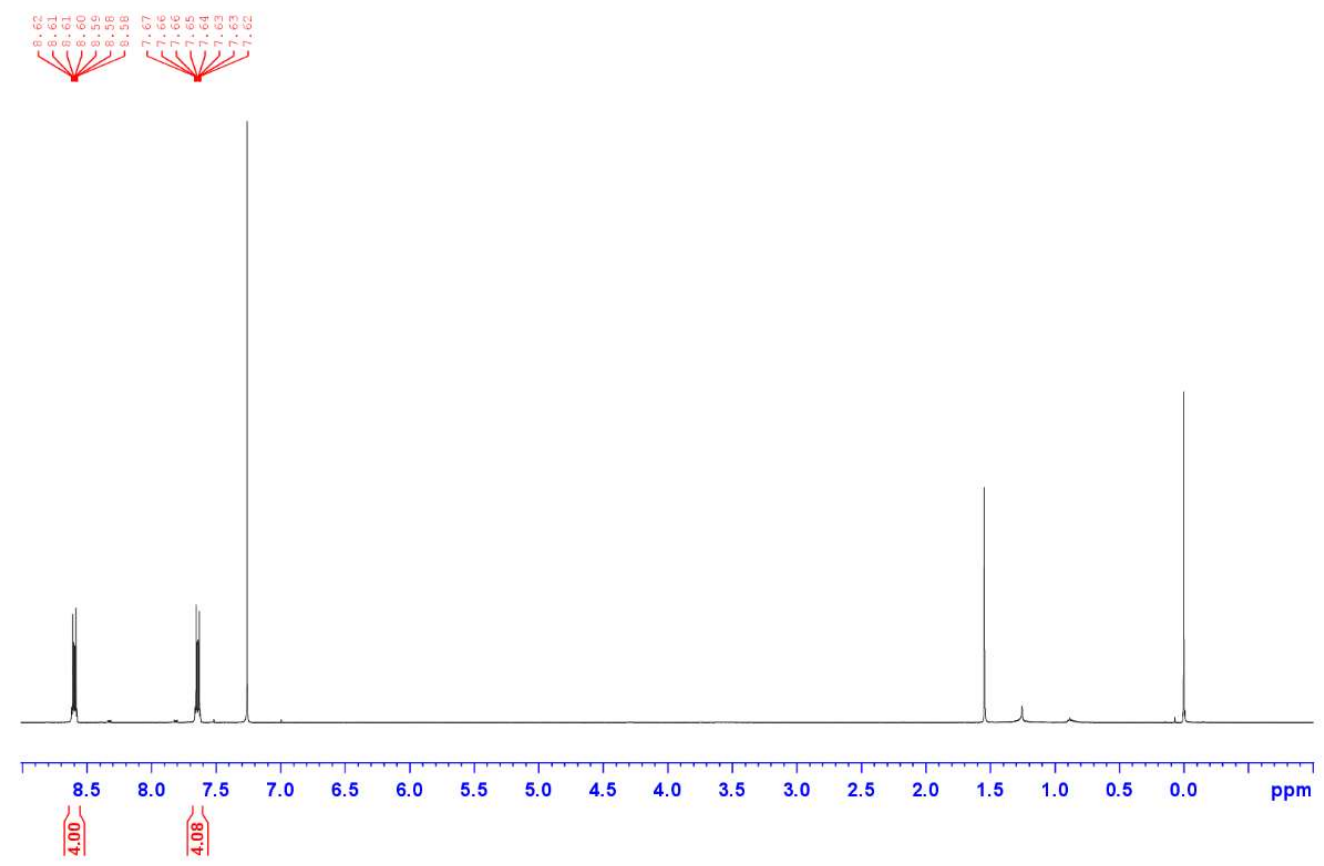

${ }^{13} \mathrm{C}$ NMR (1n- $\left.\mathrm{Br}_{2}\right)$

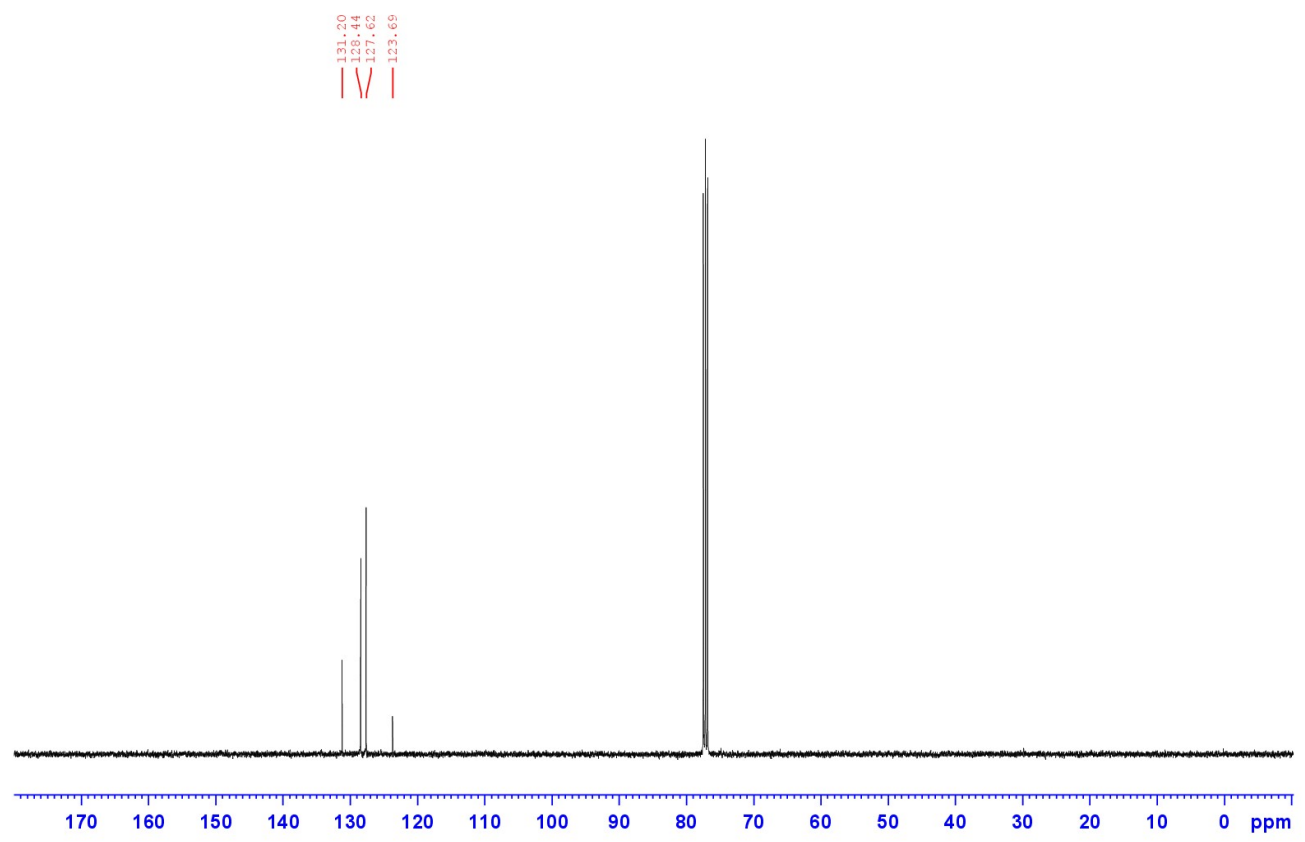


${ }^{1} \mathrm{H}$ NMR (10-Cl)

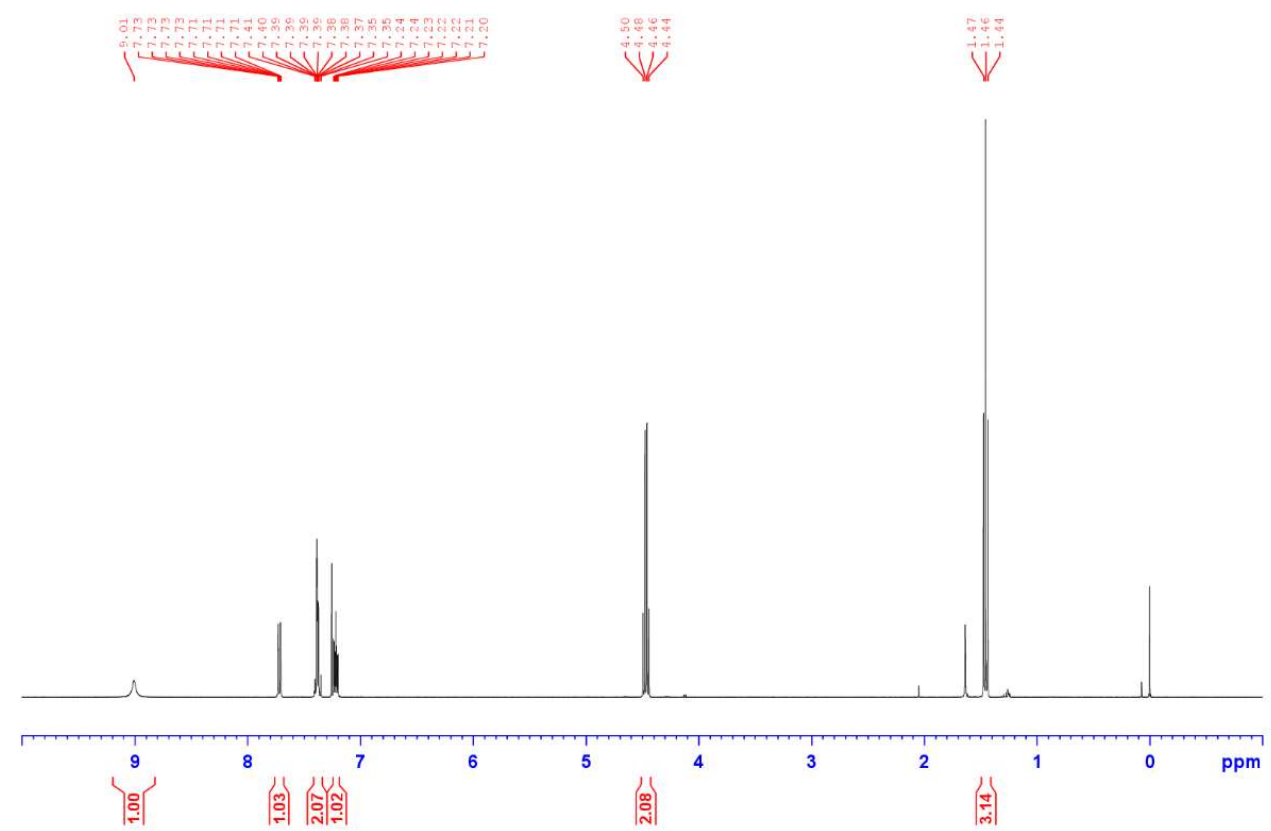

${ }^{13} \mathrm{C}$ NMR (1o-Cl)

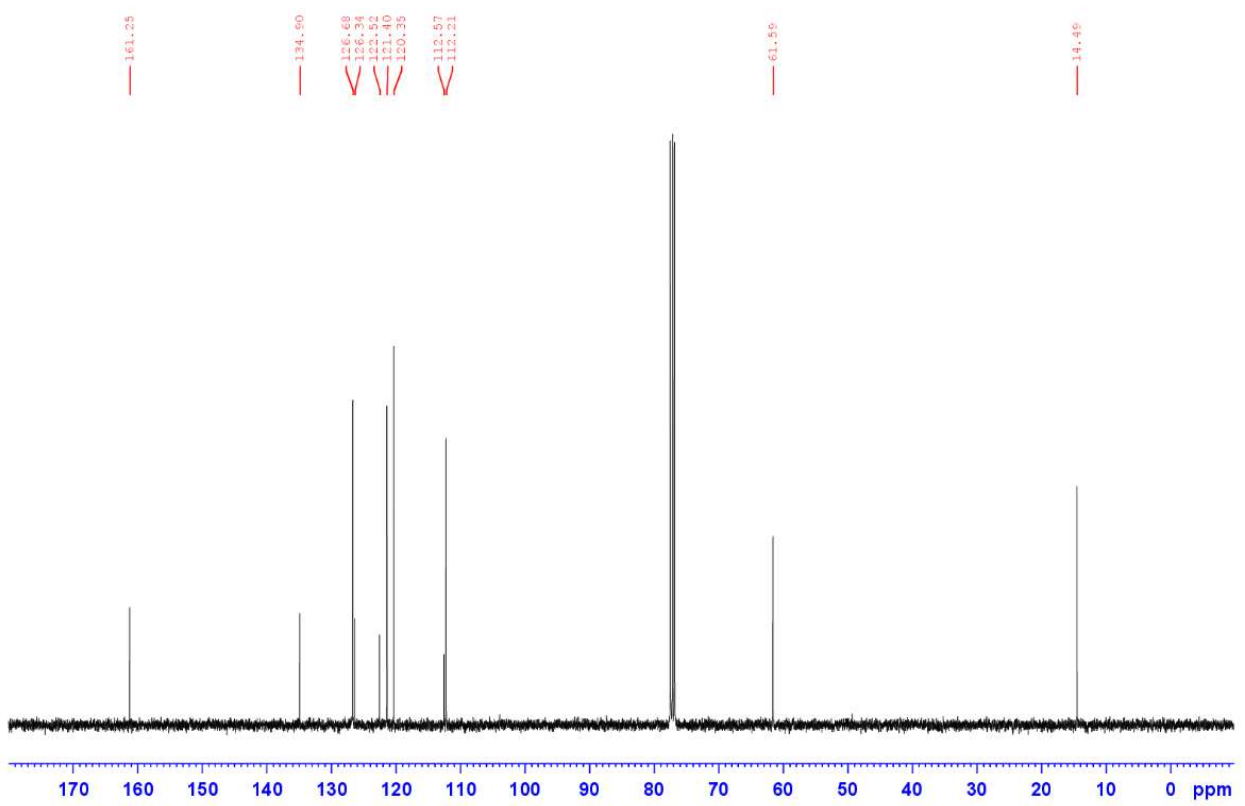


${ }^{1} \mathrm{H}$ NMR (1p-Br)

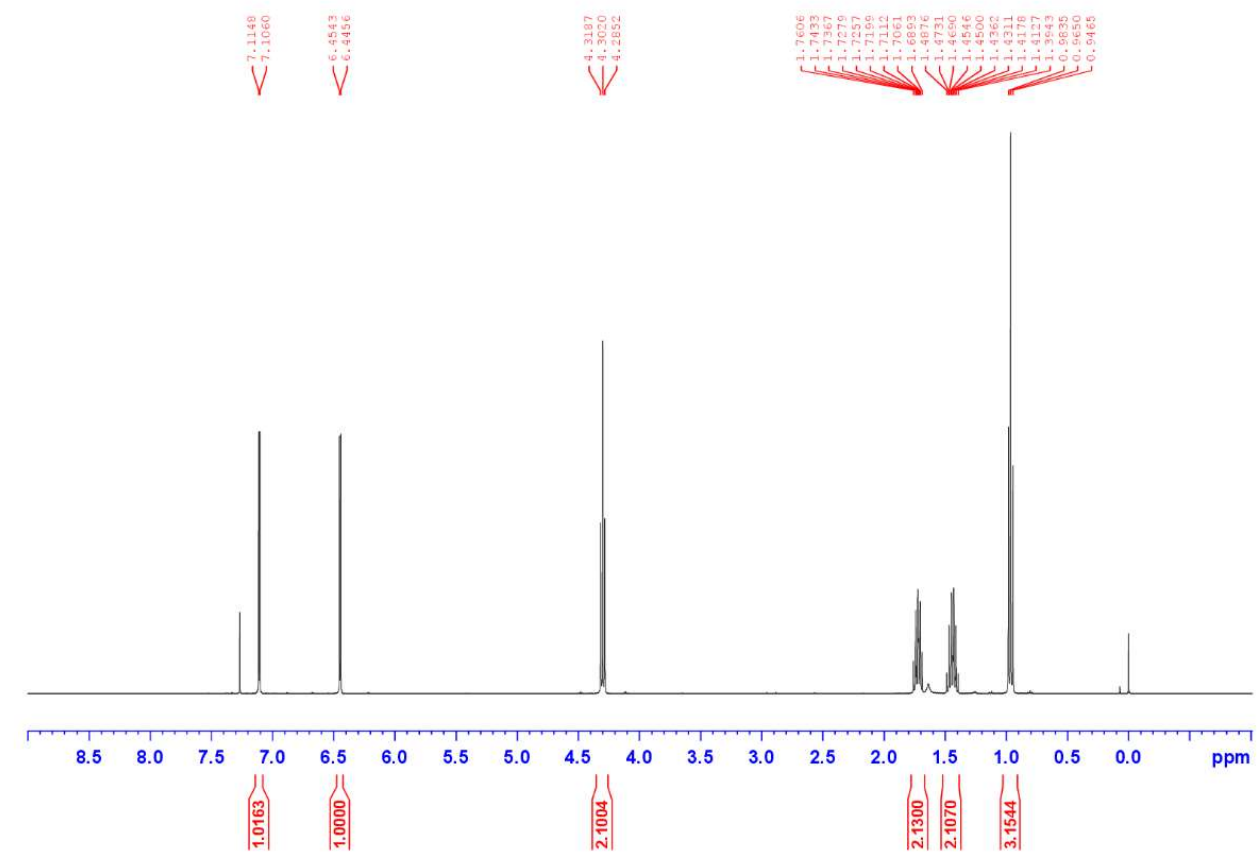

${ }^{13} \mathrm{C}$ NMR (1p-Br)

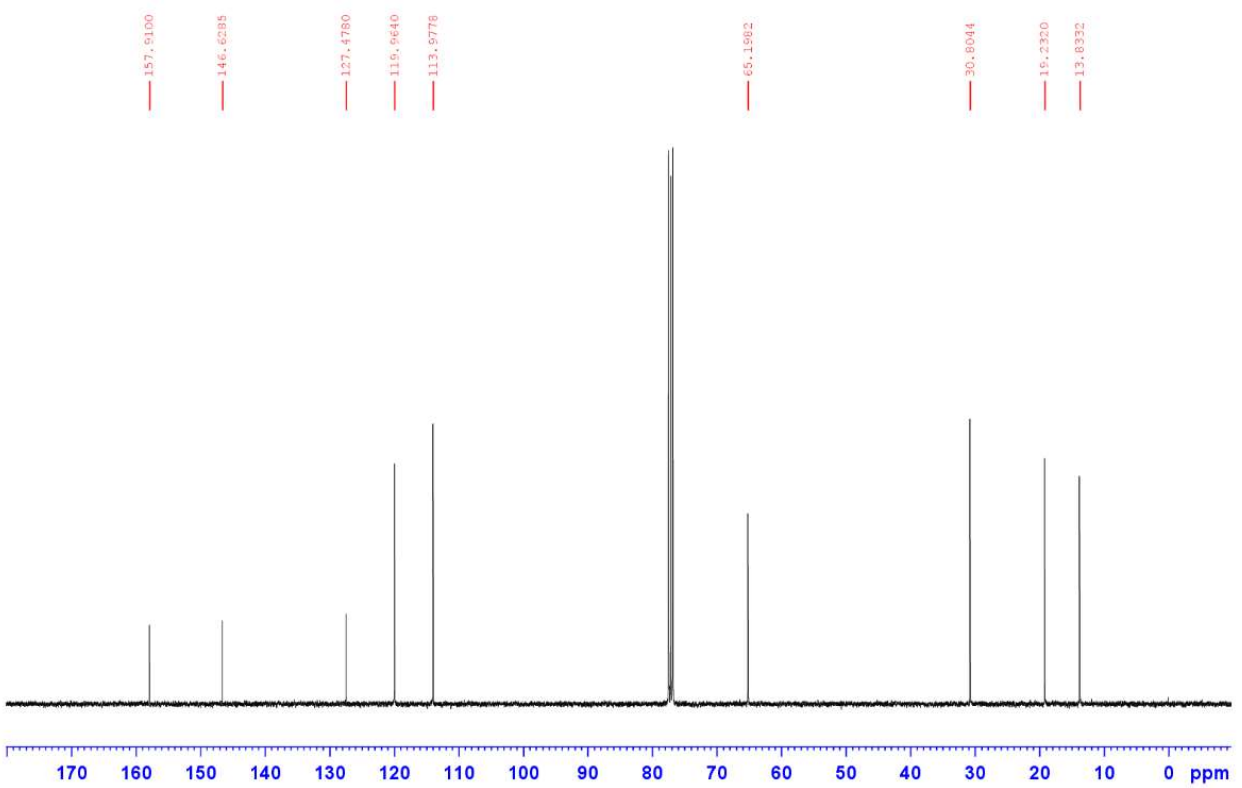


${ }^{1} \mathrm{H}$ NMR (1q-Cl)

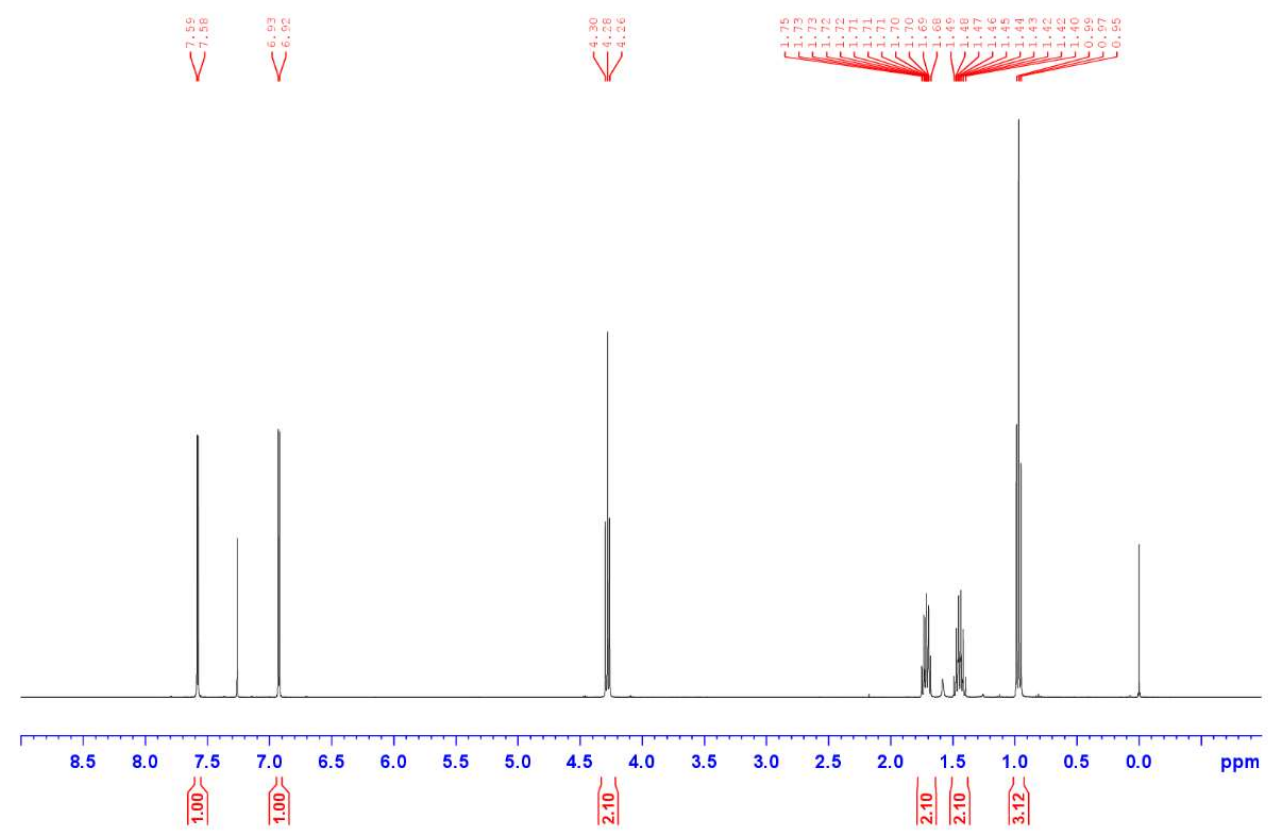

${ }^{13} \mathrm{C}$ NMR $(\mathbf{1 q}-\mathrm{Cl})$

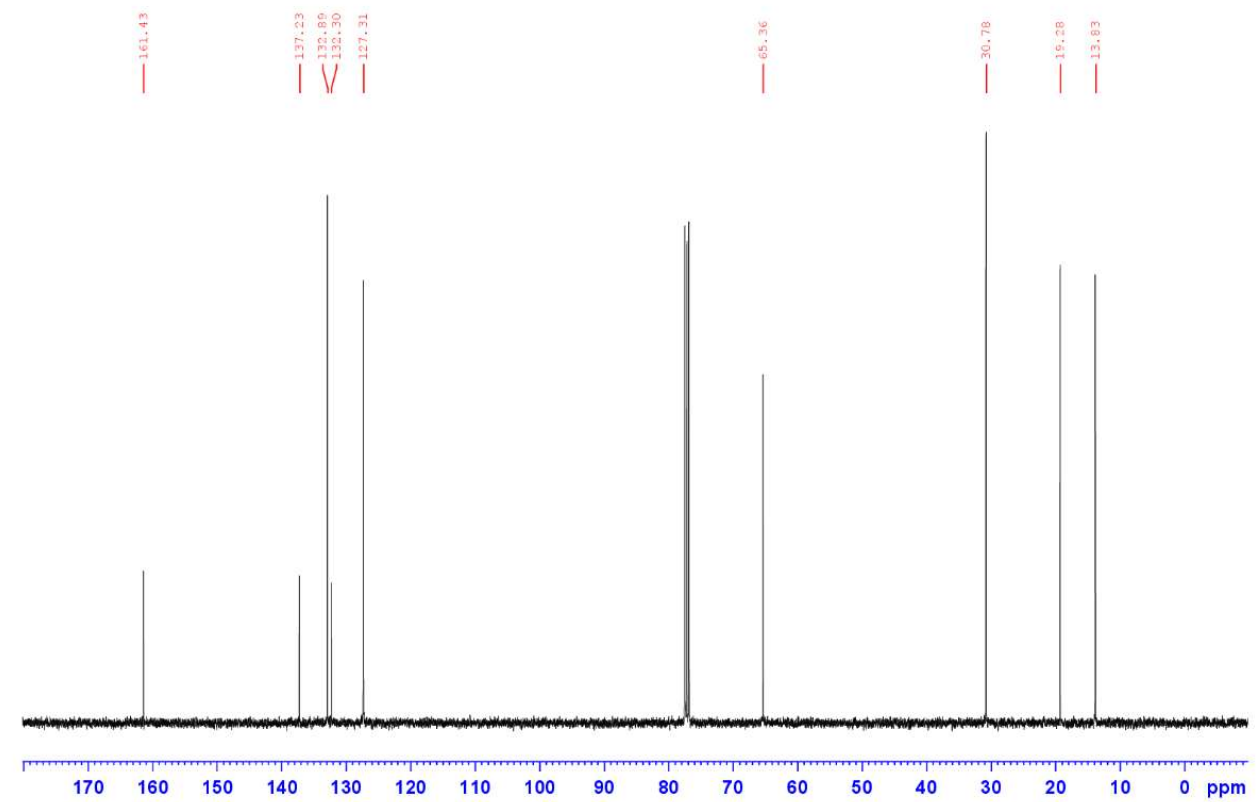


${ }^{1} \mathrm{H}$ NMR (1r-Br)
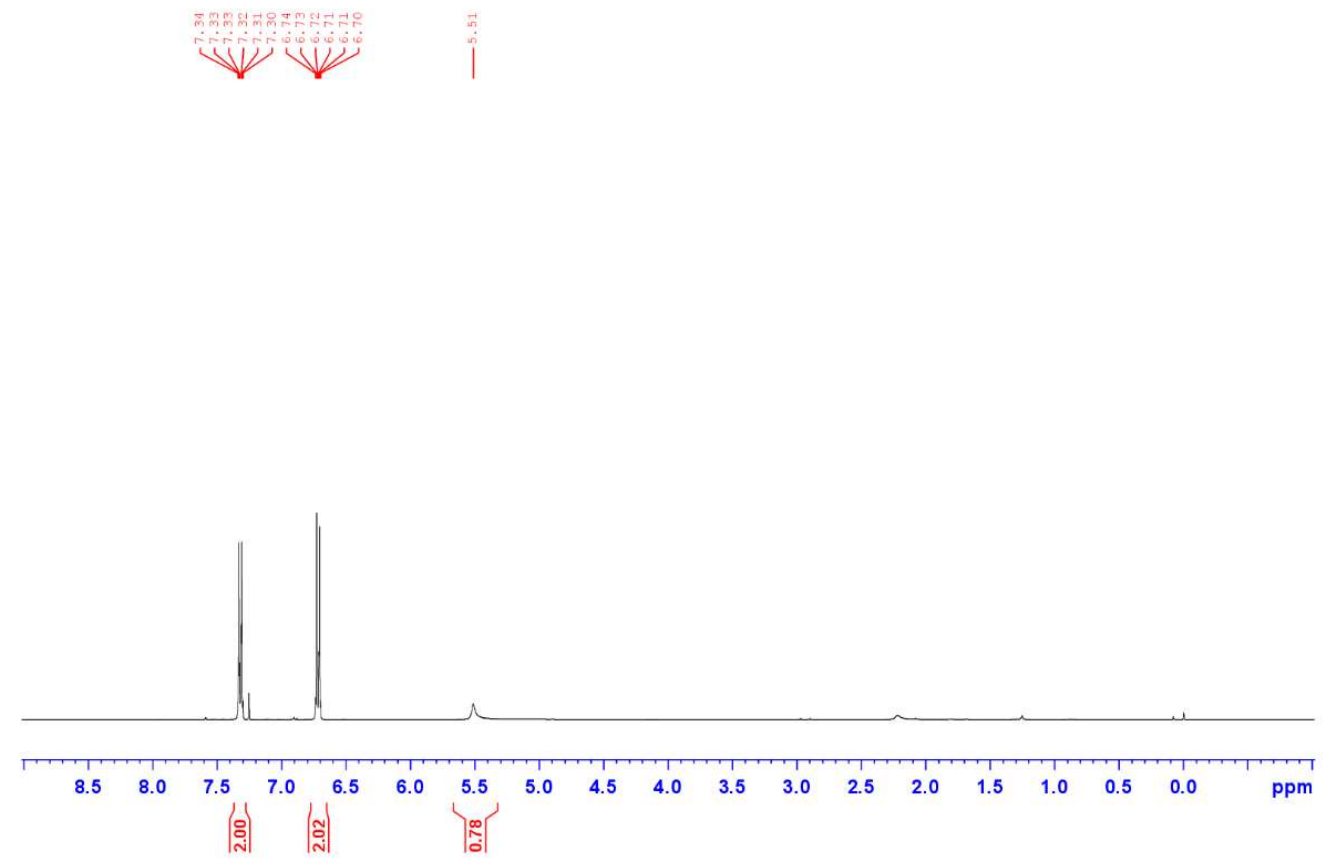

${ }^{13} \mathrm{C}$ NMR (1r-Br)

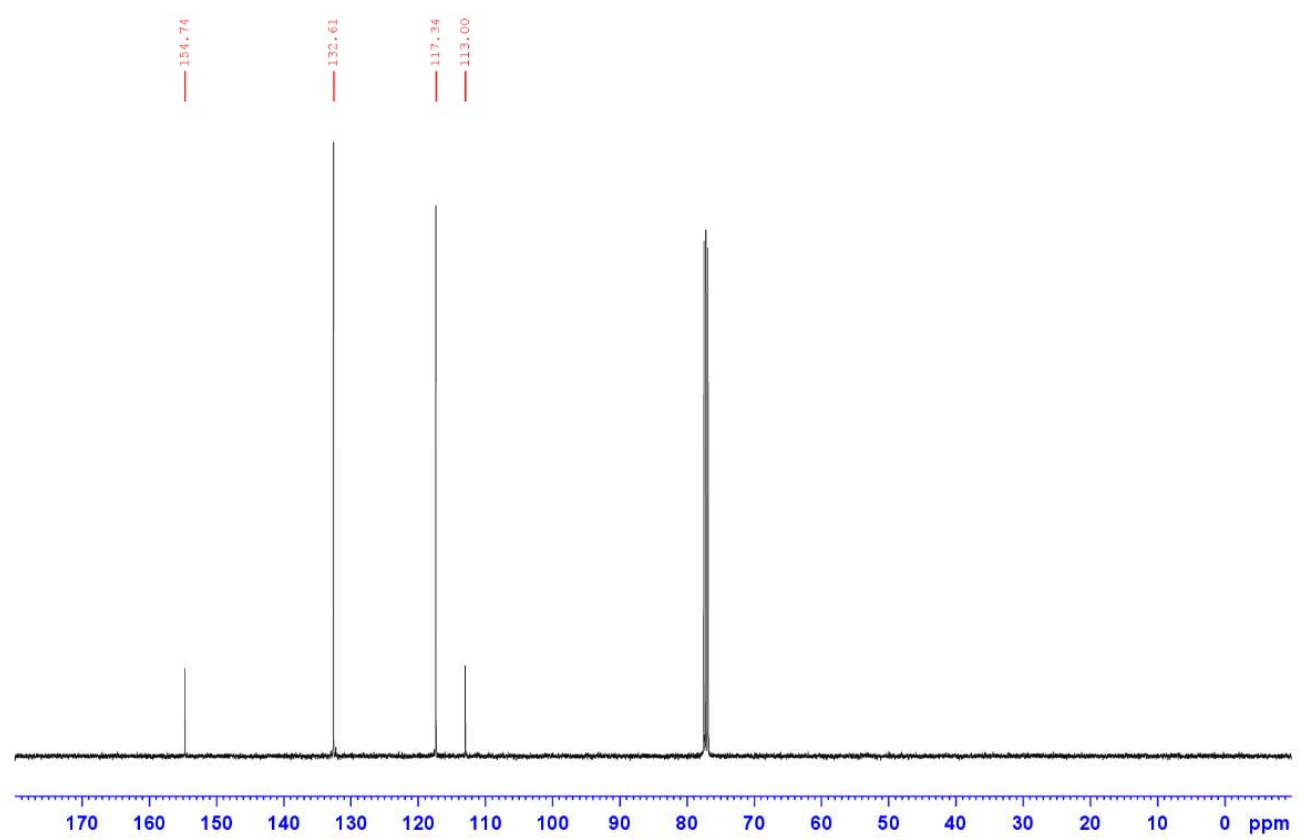


${ }^{1} \mathrm{H}$ NMR (1s-Br)

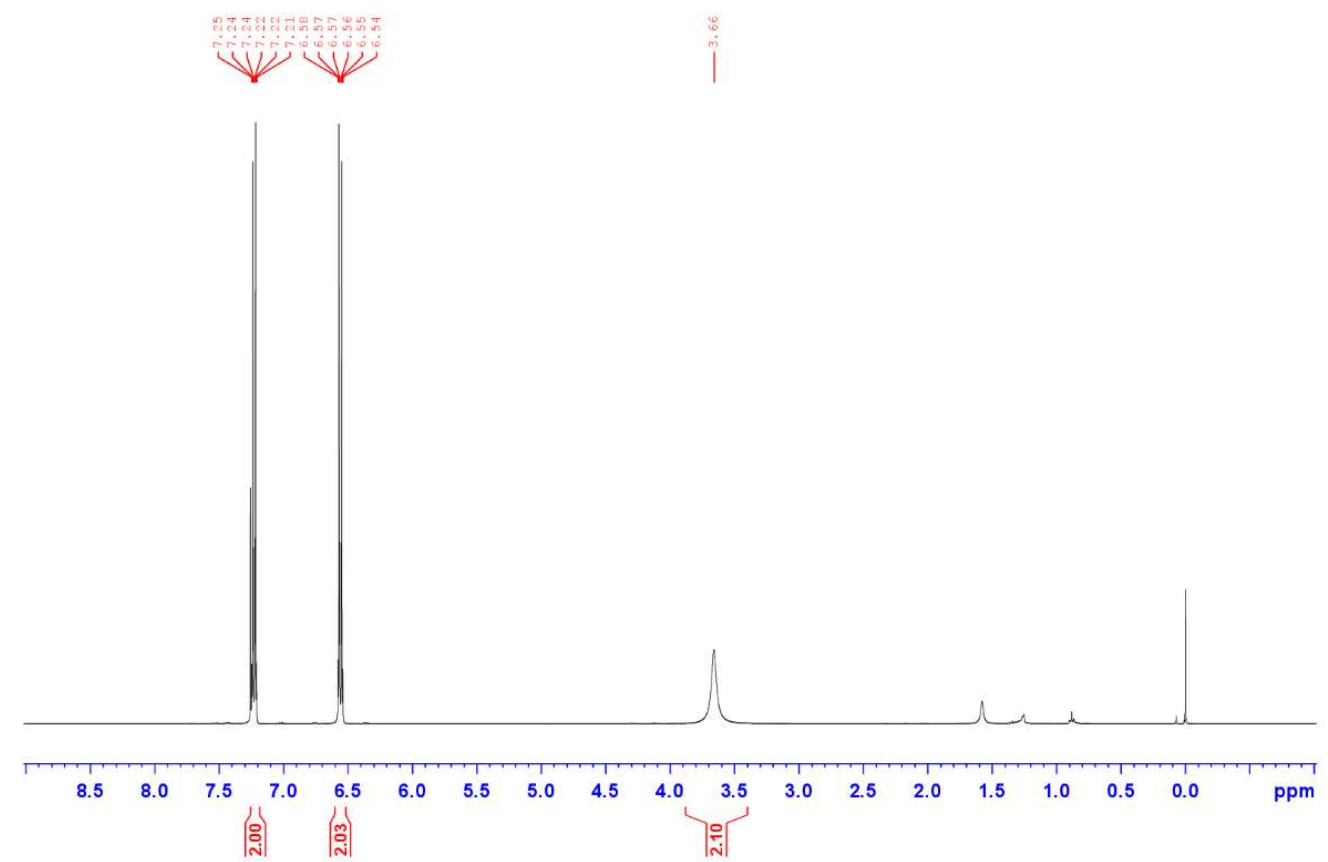

${ }^{13} \mathrm{C}$ NMR (1s-Br)

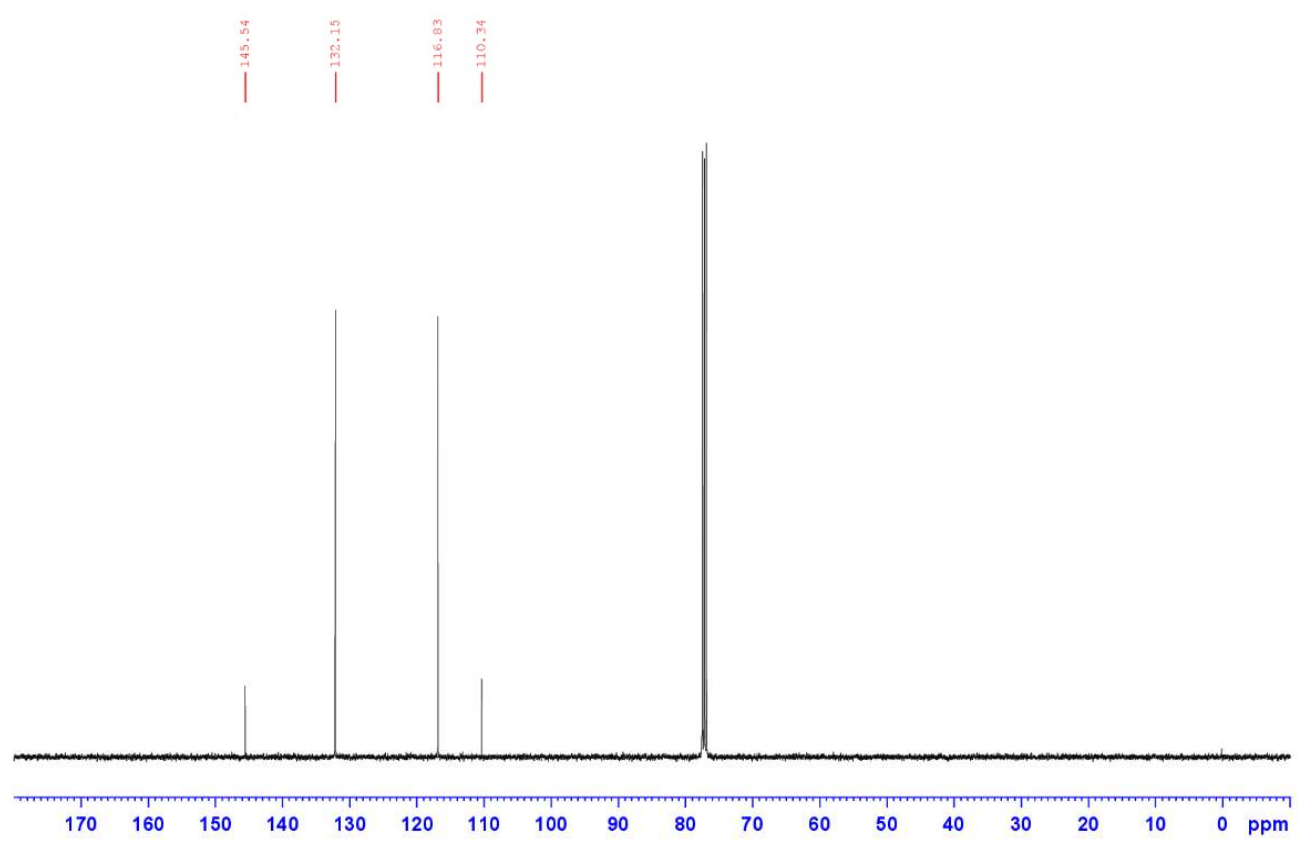


${ }^{1} \mathrm{H}$ NMR (1t-Cl)

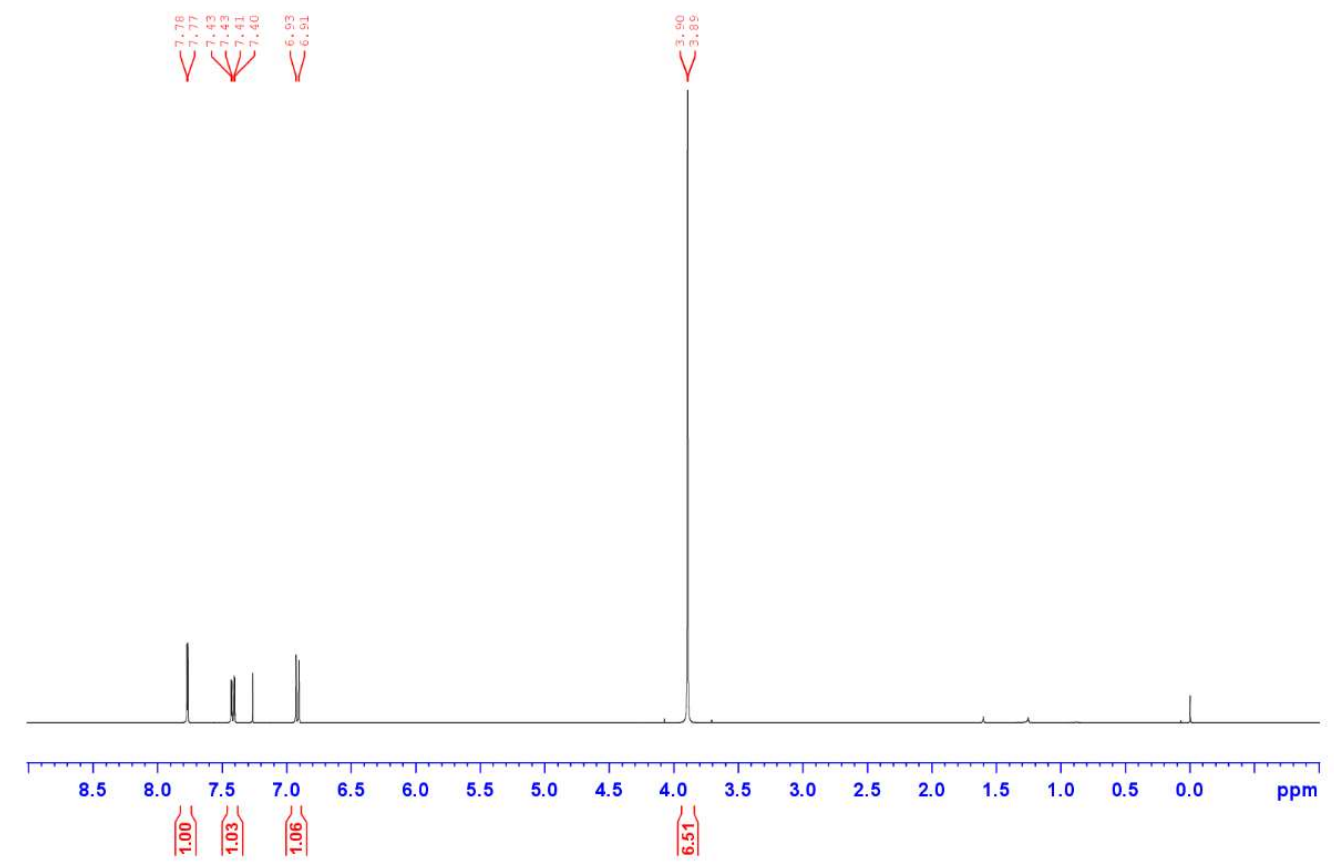

${ }^{13} \mathrm{C}$ NMR (1t-Cl)

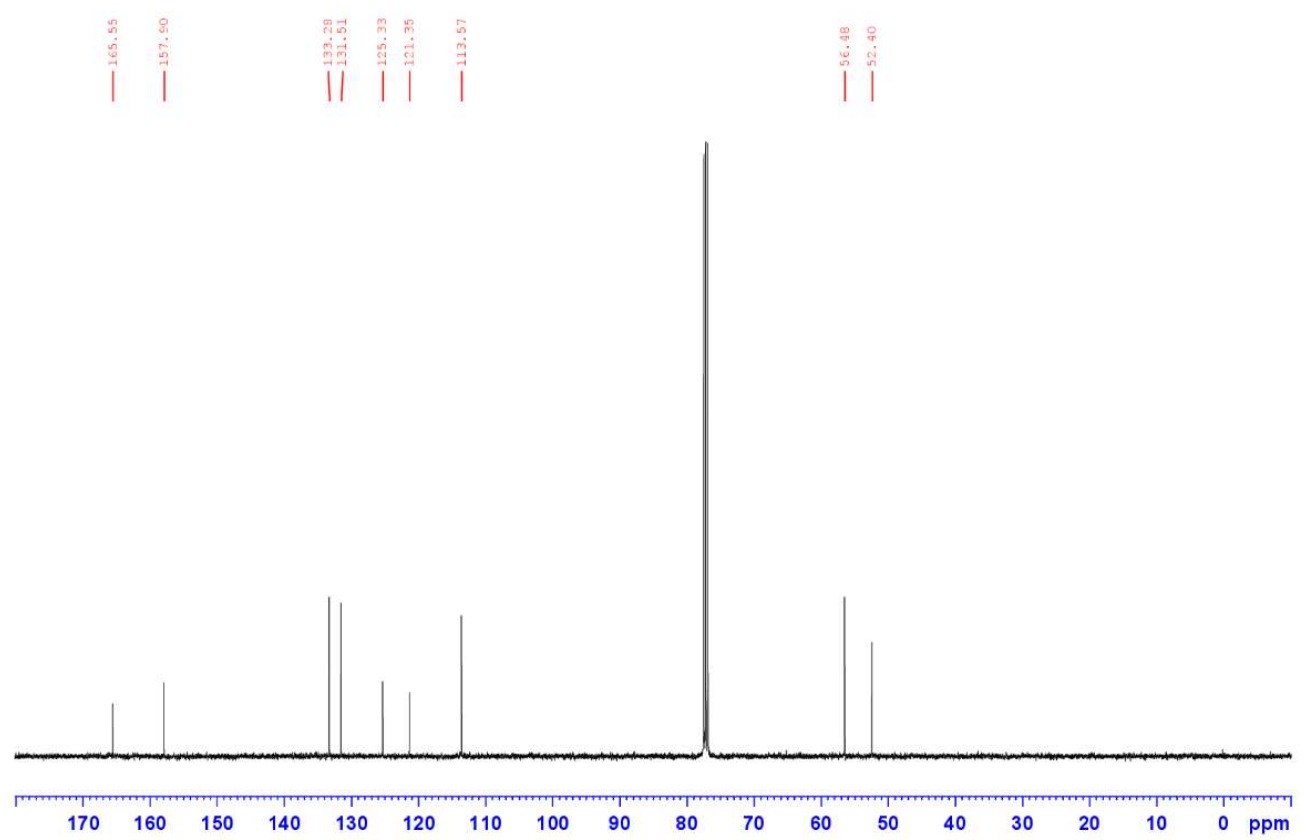


${ }^{1} \mathrm{H}$ NMR (1t-Br)

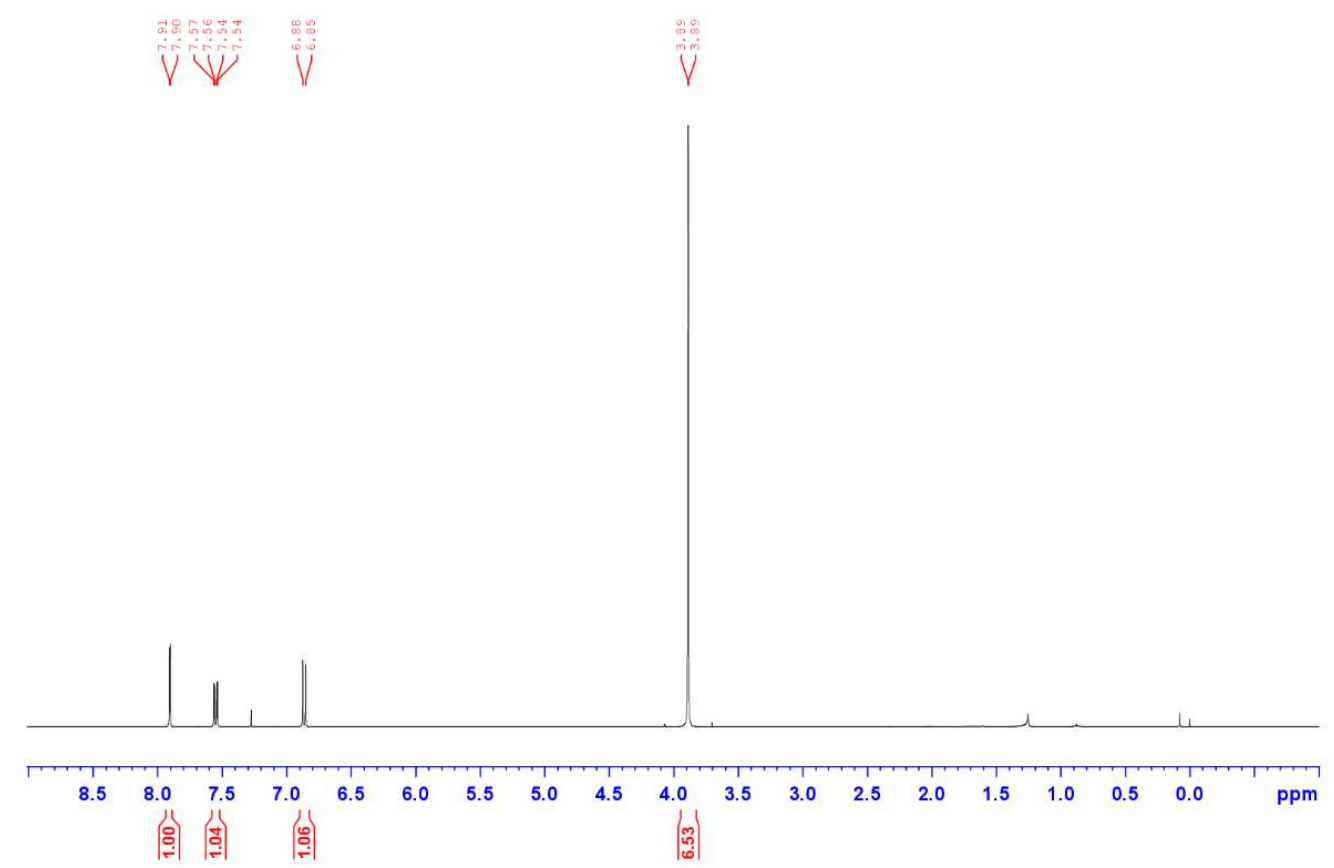

${ }^{13} \mathrm{C}$ NMR (1t-Br)

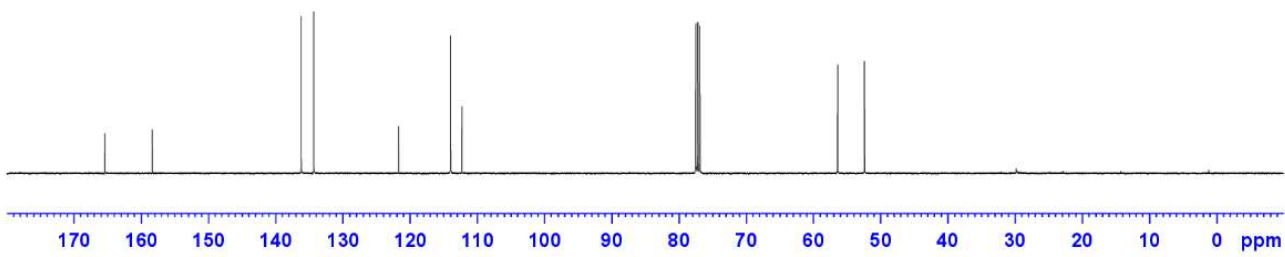


${ }^{1} \mathrm{H}$ NMR (1u-Br)

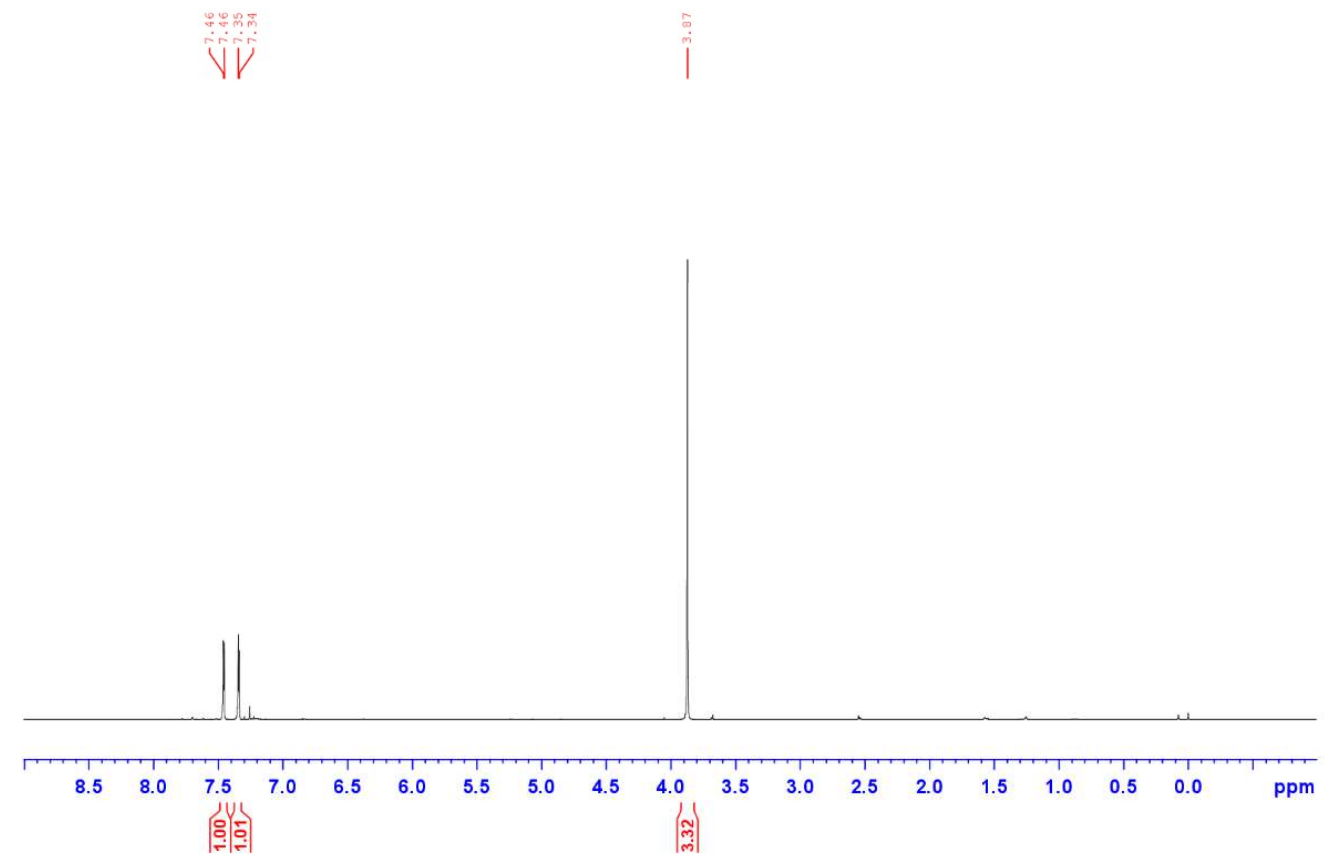

${ }^{13} \mathrm{C}$ NMR (1u-Br)

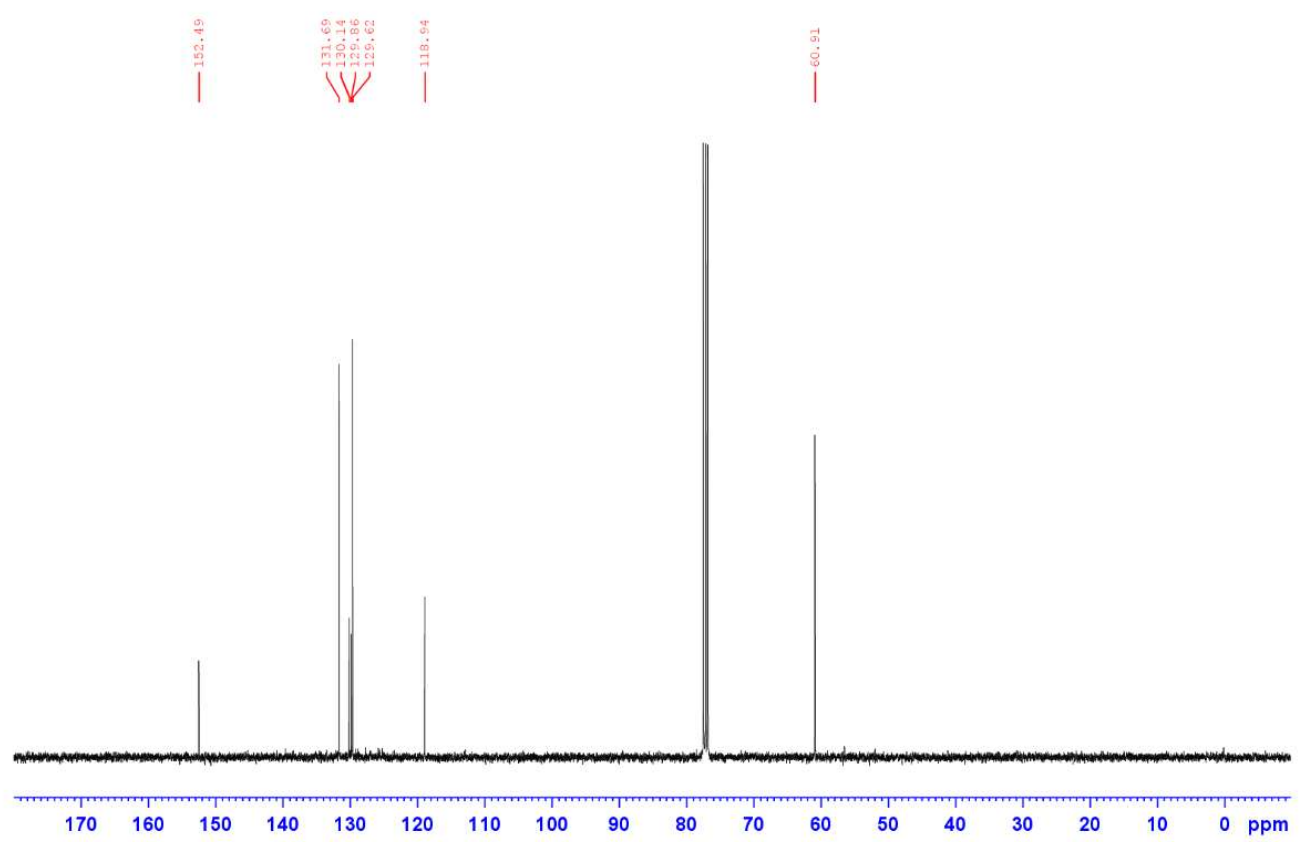


${ }^{1} \mathrm{H}$ NMR (1v-Br)

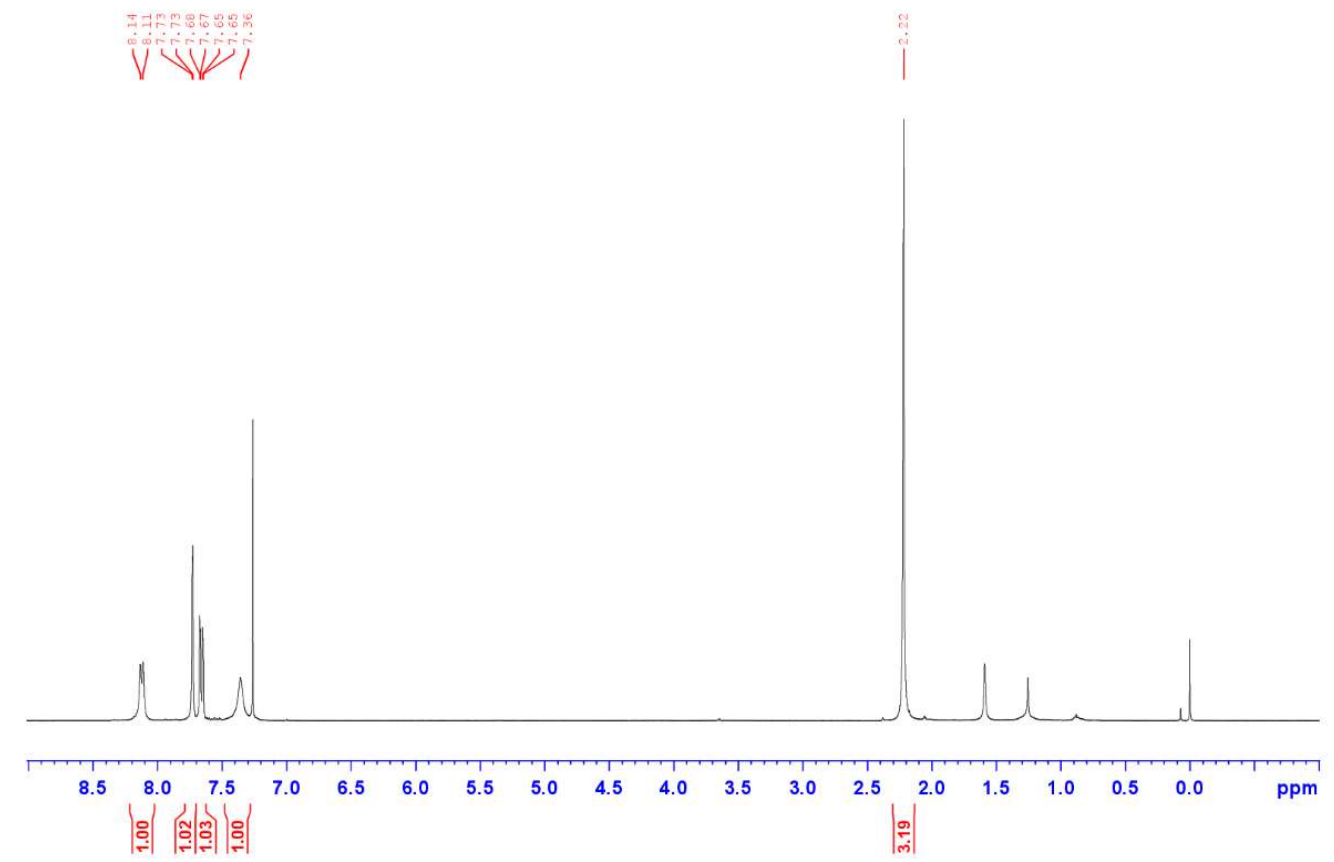

${ }^{13} \mathrm{C}$ NMR (1v-Br)

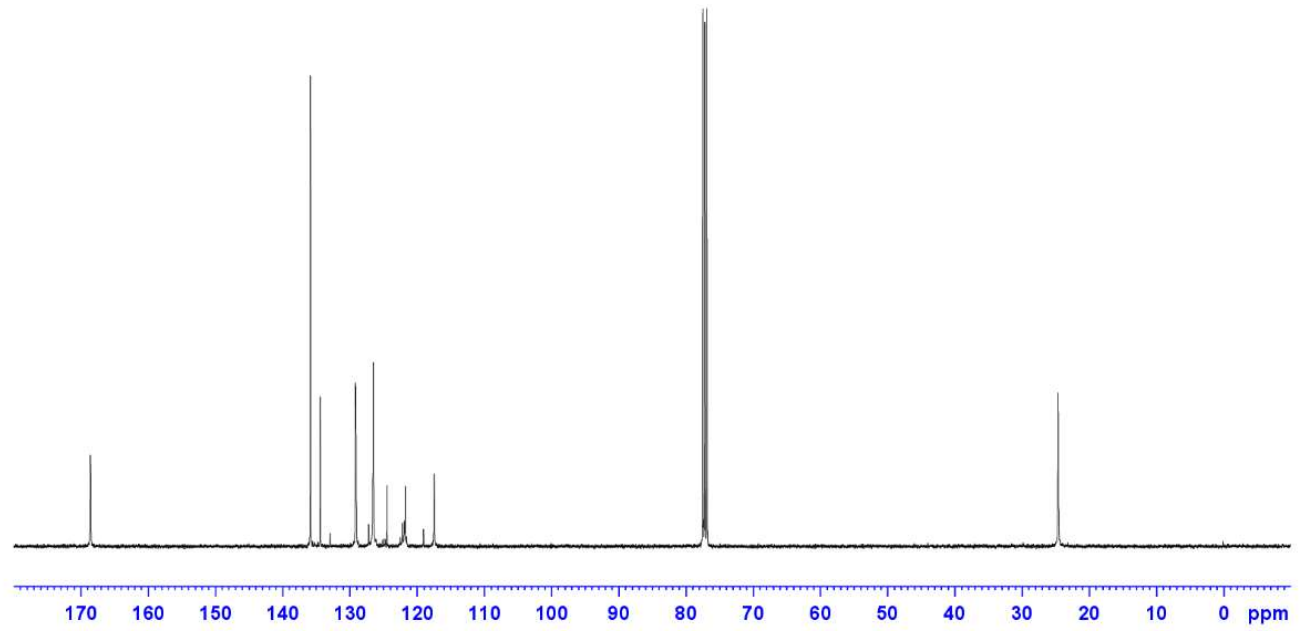


${ }^{19}$ F NMR (1v-Br)

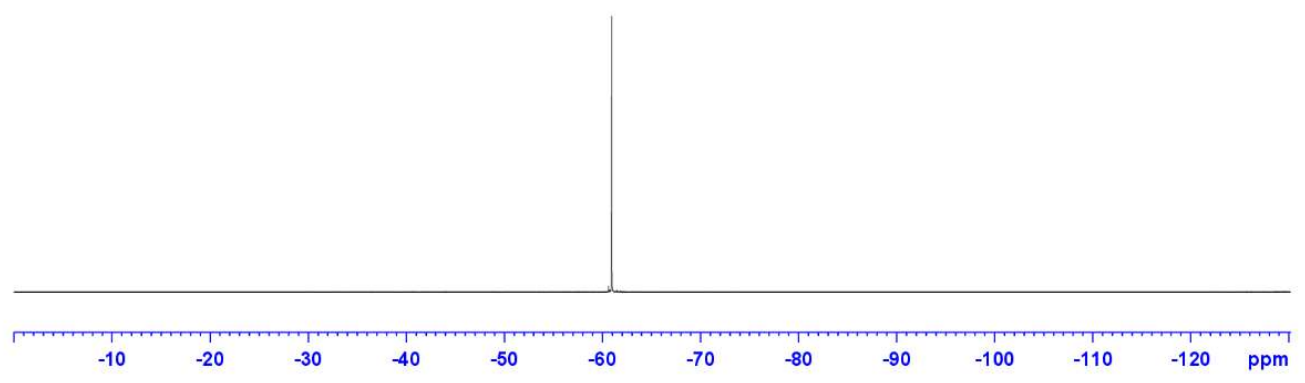


${ }^{1} \mathrm{H}$ NMR (1w-Br)

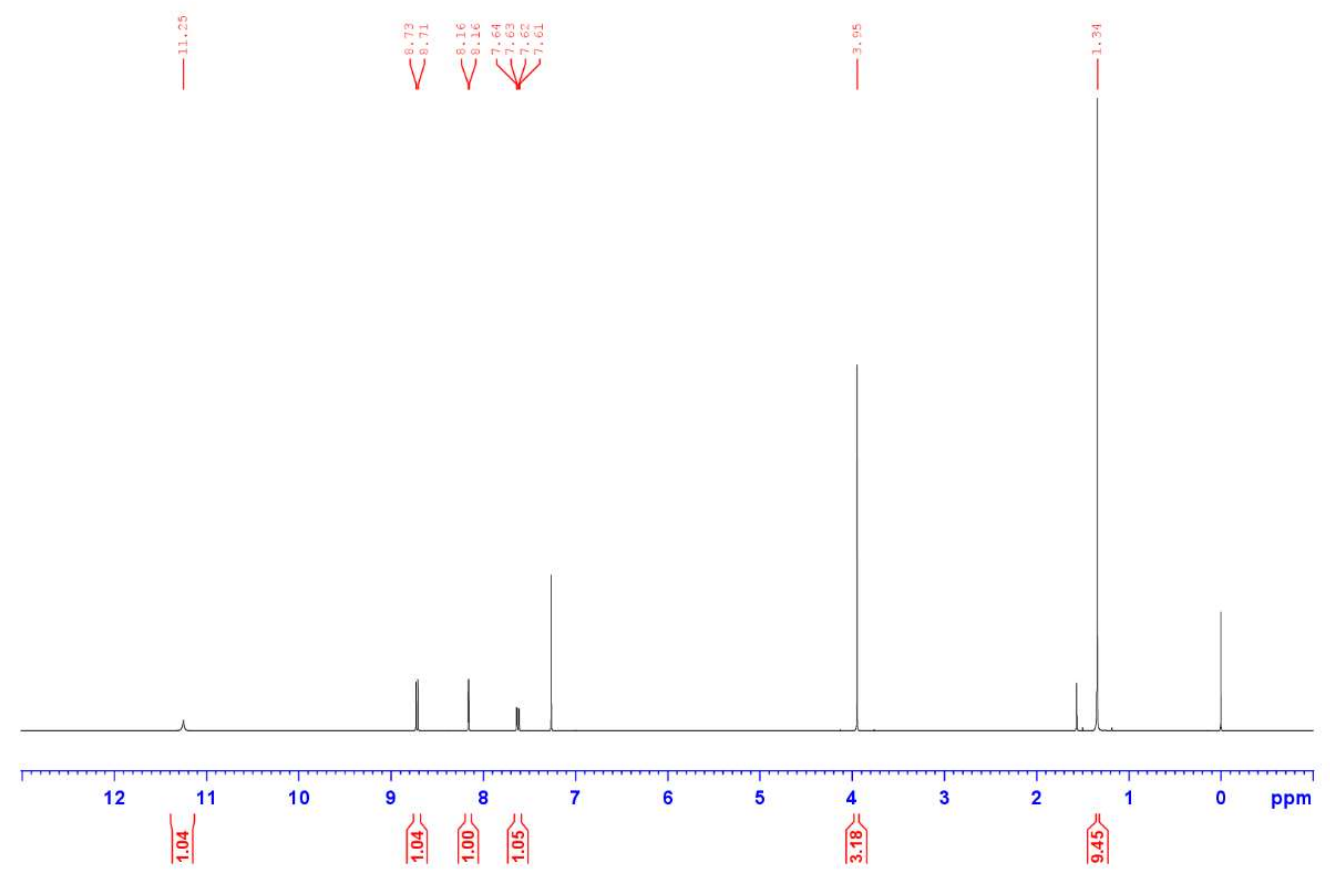

${ }^{13} \mathrm{C}$ NMR (1w-Br)

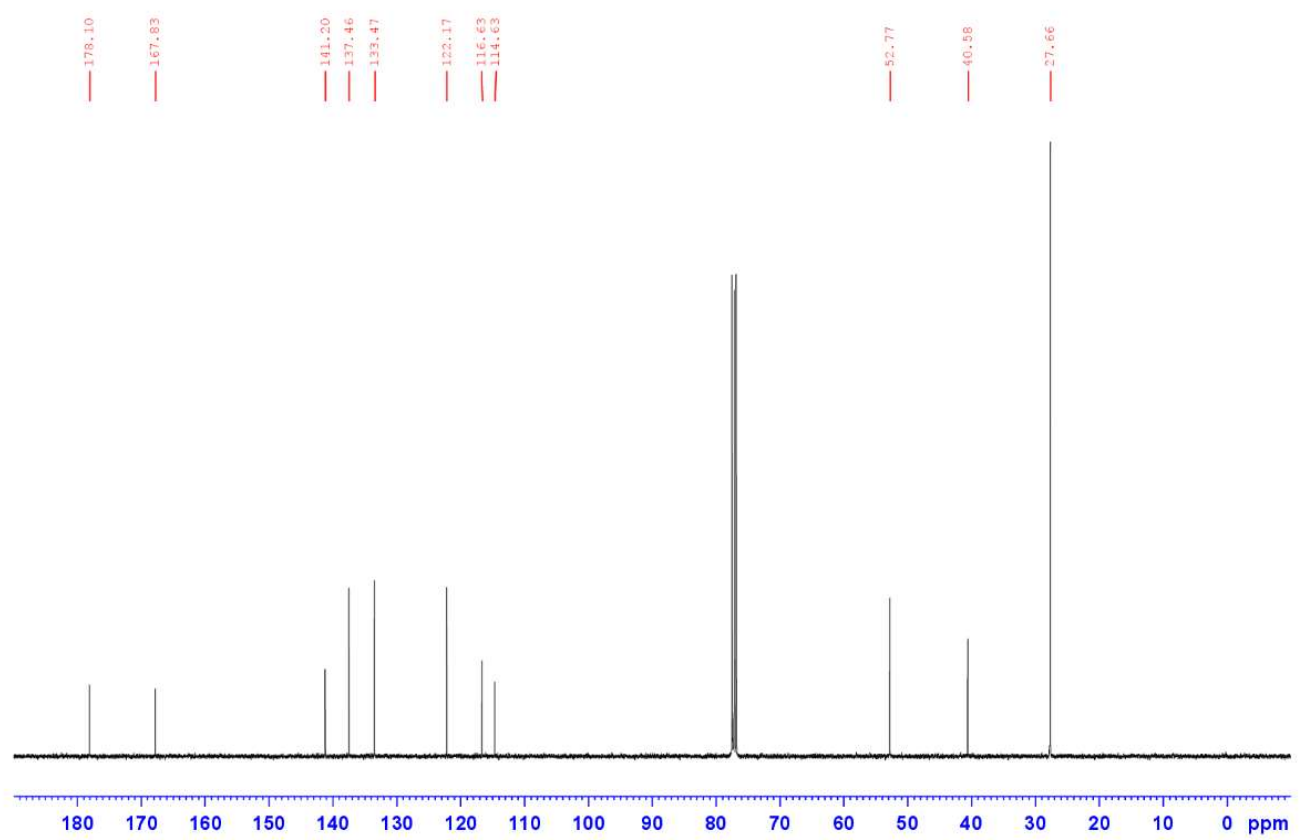




\section{${ }^{1} \mathrm{H}$ NMR (1x-Br)}

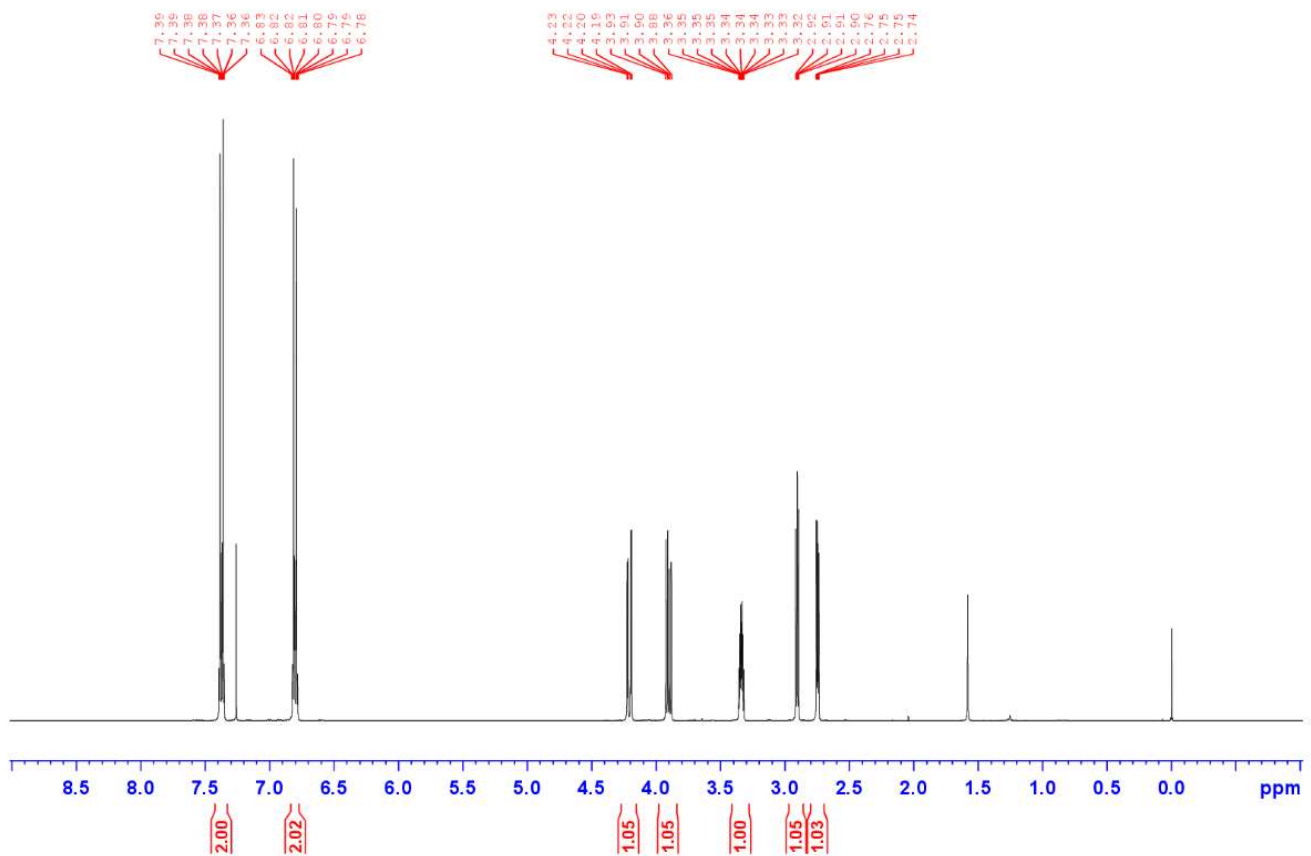

${ }^{13} \mathrm{C}$ NMR (1x-Br)

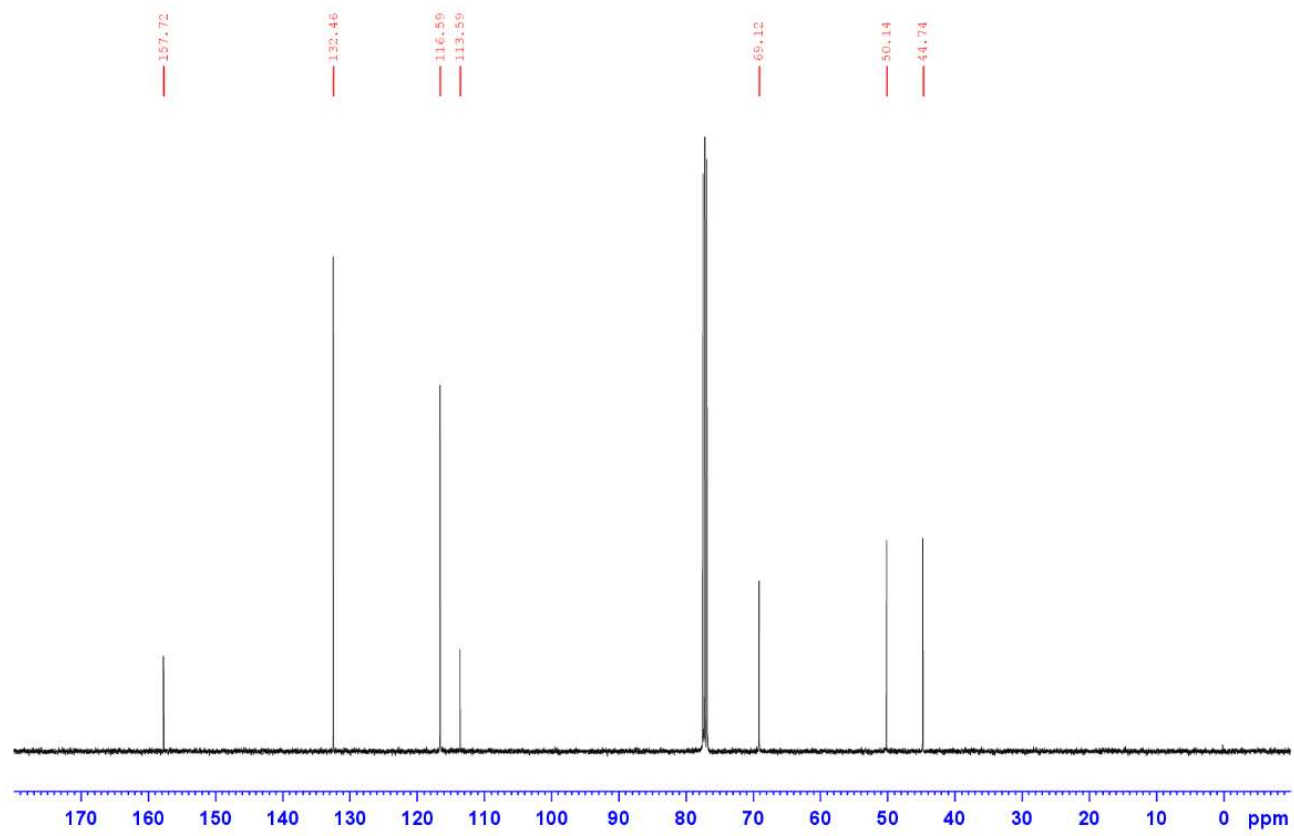


${ }^{1} \mathrm{H}$ NMR (1y-Br)

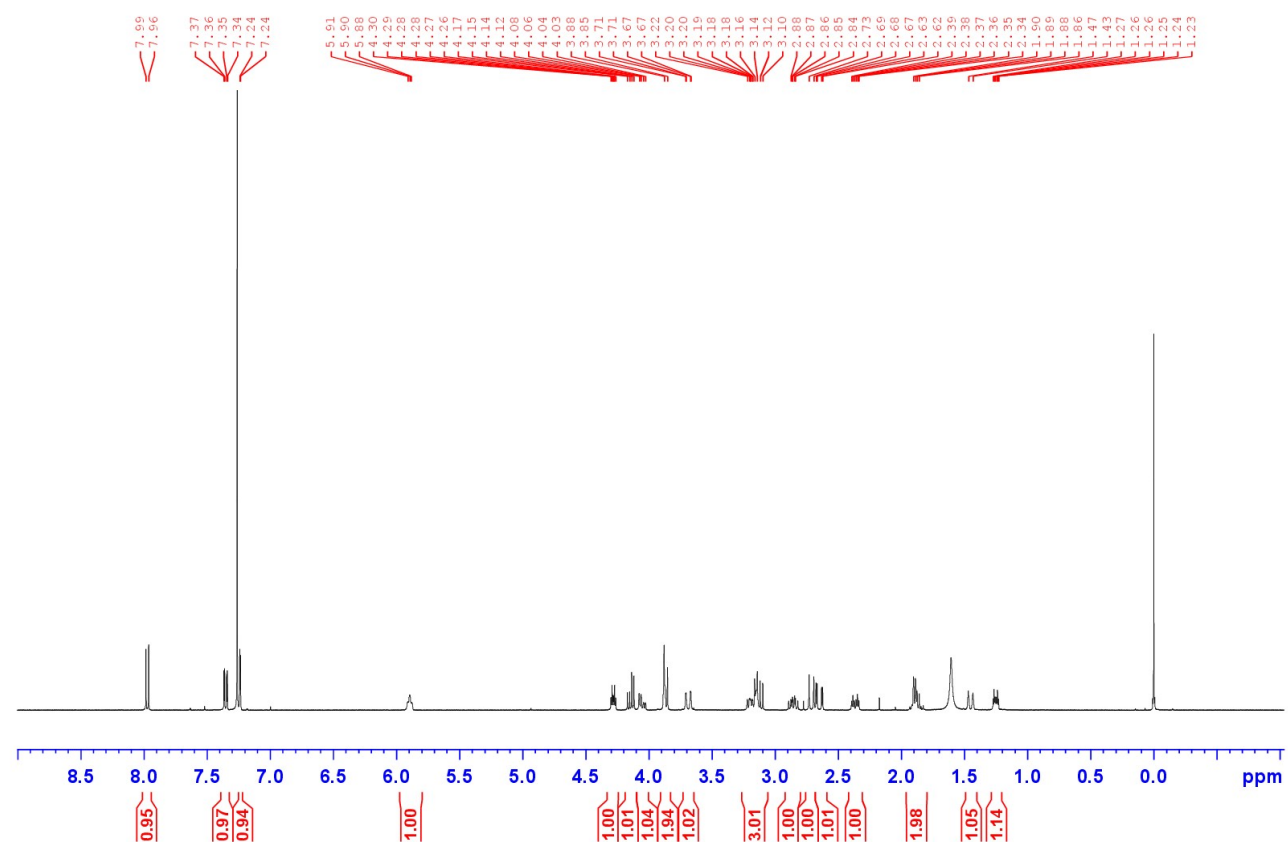

${ }^{13} \mathrm{C}$ NMR (1y-Br)

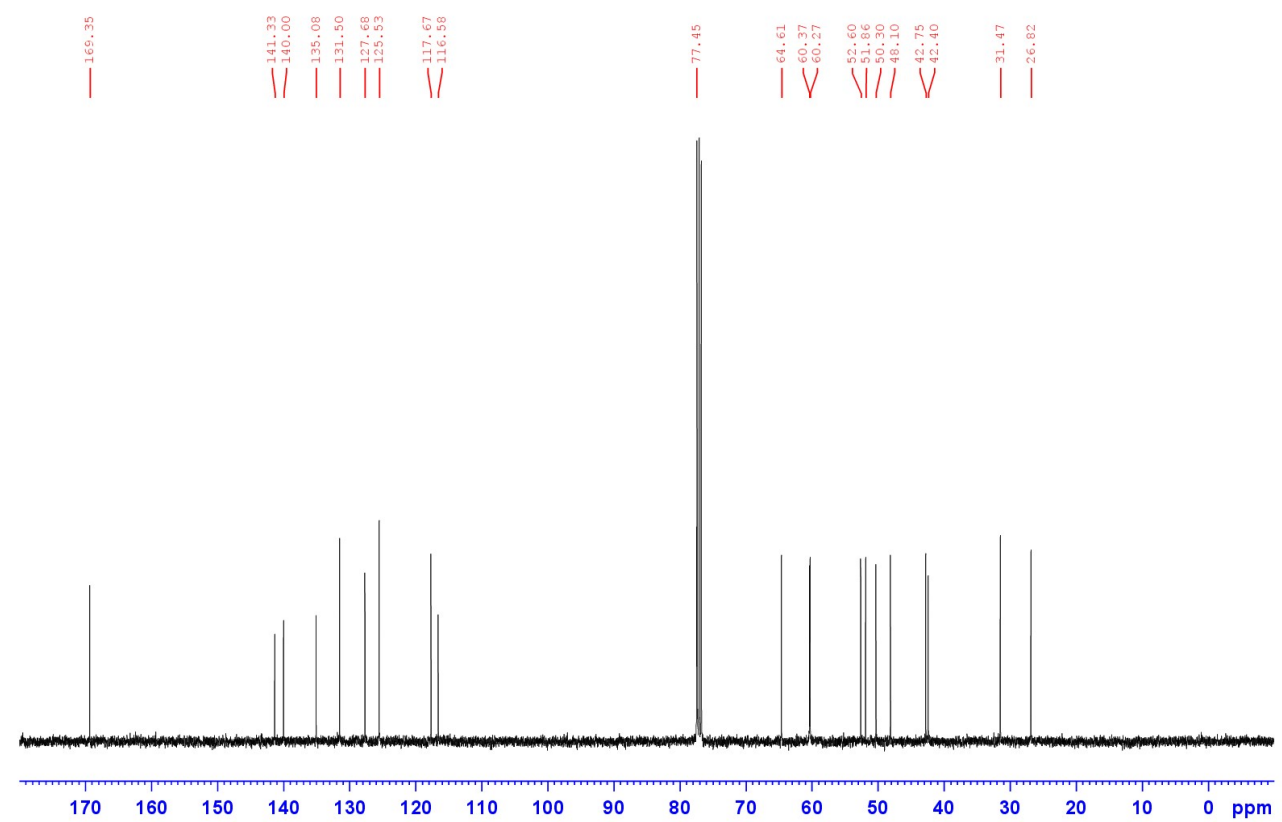


${ }^{1} \mathrm{H}$ NMR $\left(\mathbf{1} \mathbf{y}-\mathrm{Br}_{2}\right)$

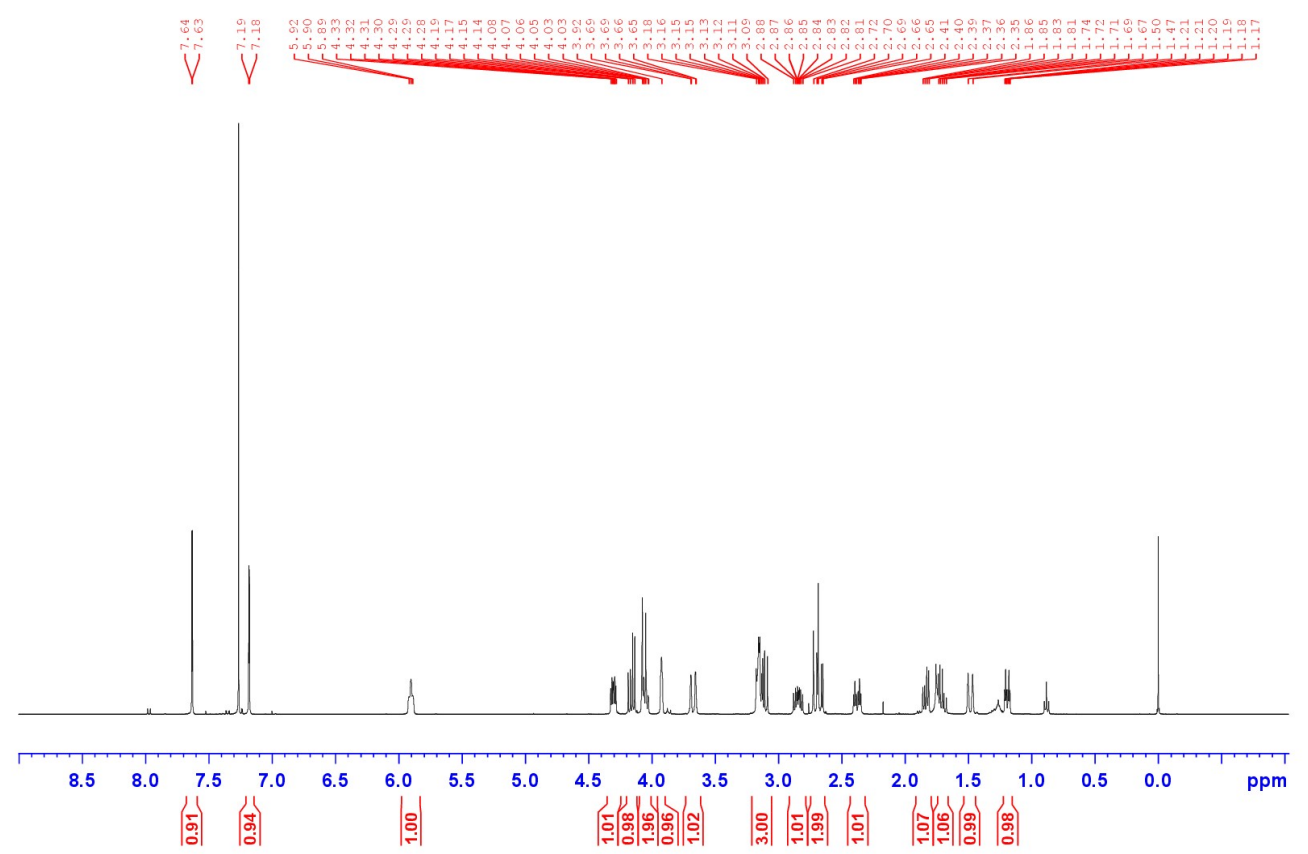

${ }^{13} \mathrm{C}$ NMR (1y-Br $)$

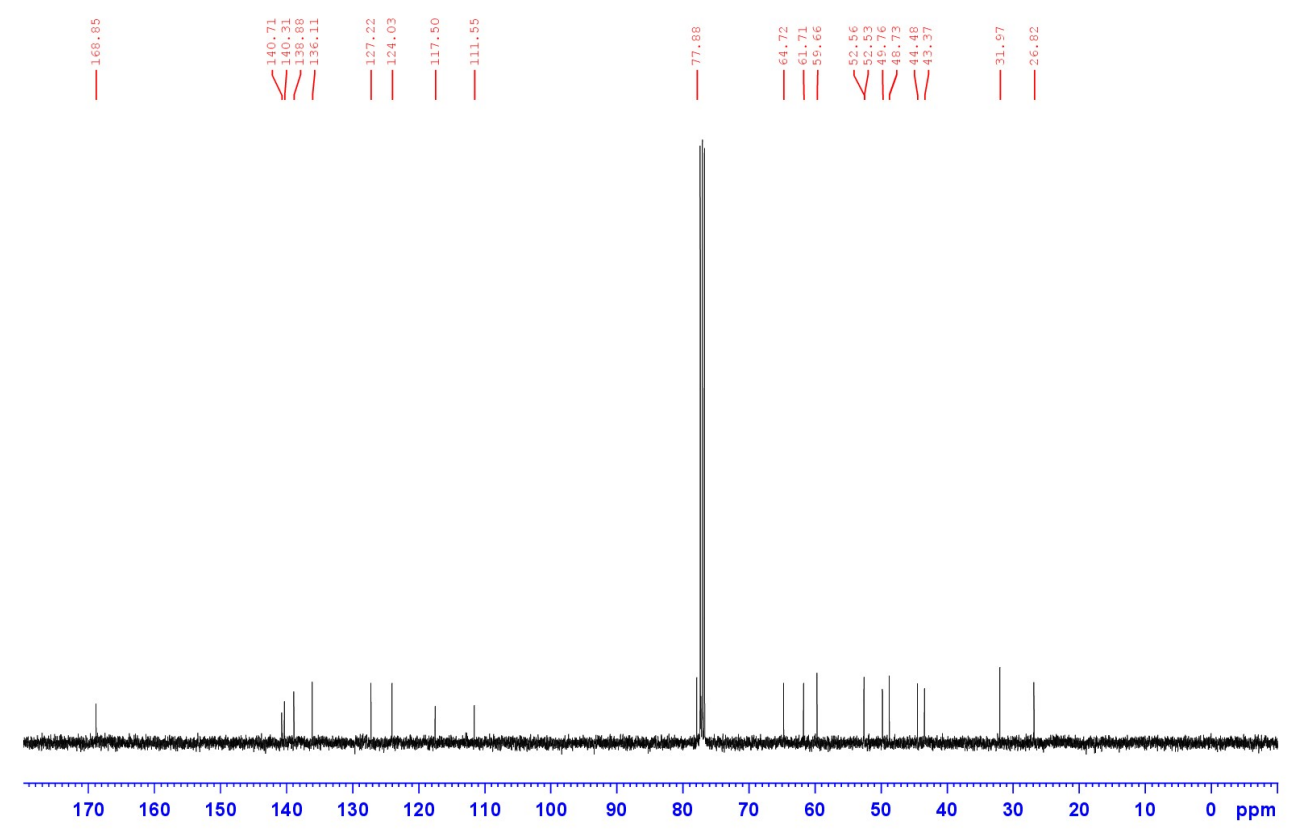


${ }^{1} \mathrm{H}$ NMR (Trip-SMe-Br3, major isomer)
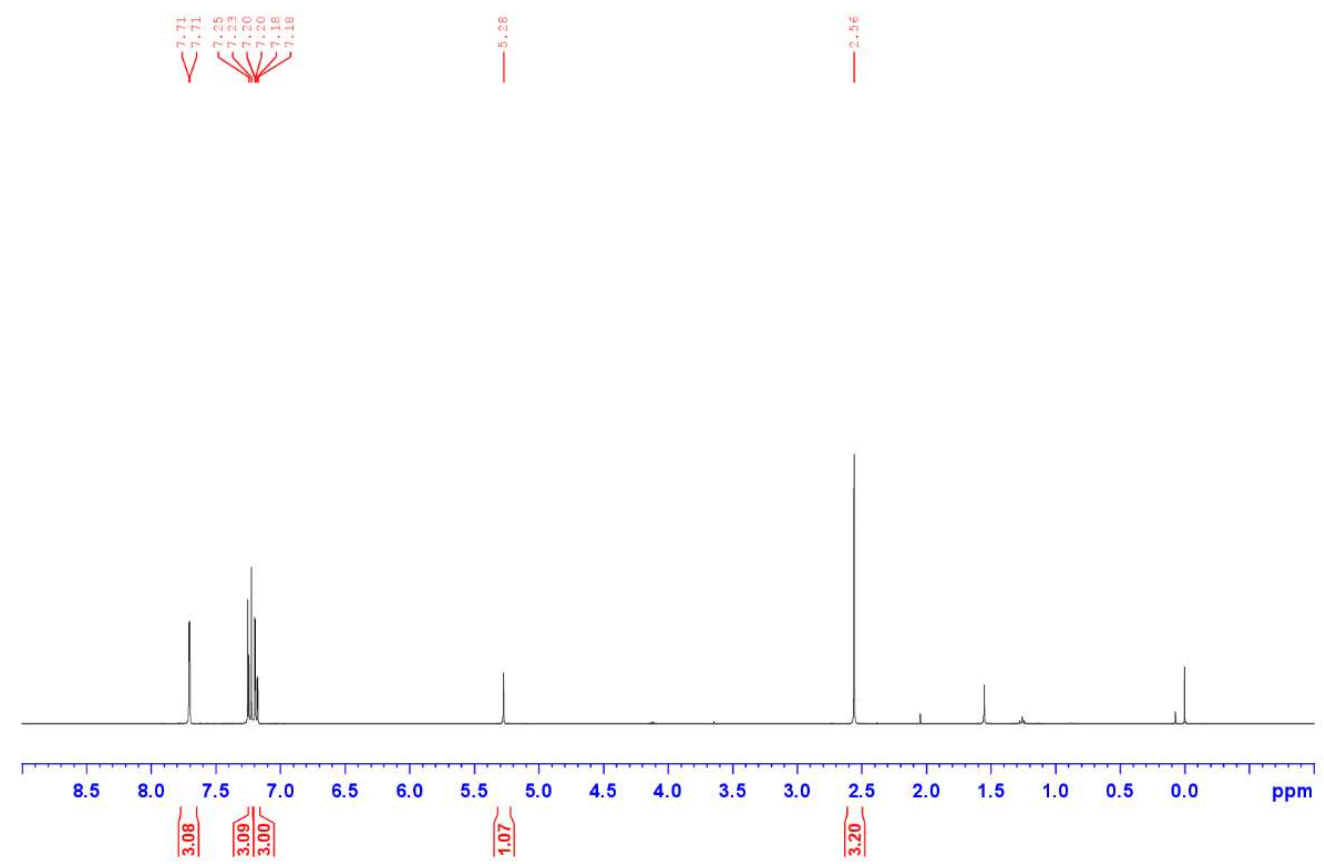

${ }^{13} \mathrm{C}$ NMR (Trip-SMe-Br3 3 , major isomer)

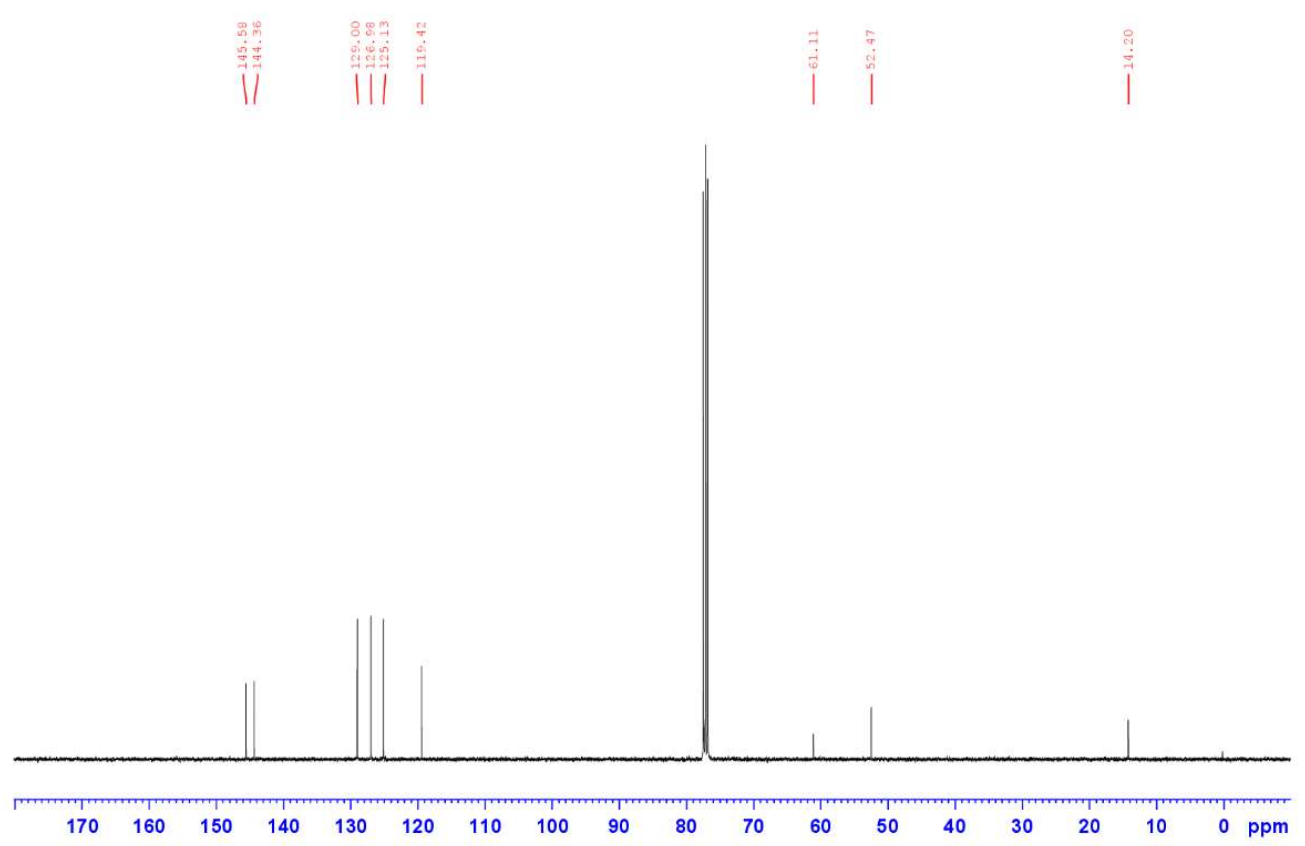


${ }^{1} \mathrm{H}$ NMR (Trip-SMe- $\mathrm{Br}_{3}$, minor isomer)

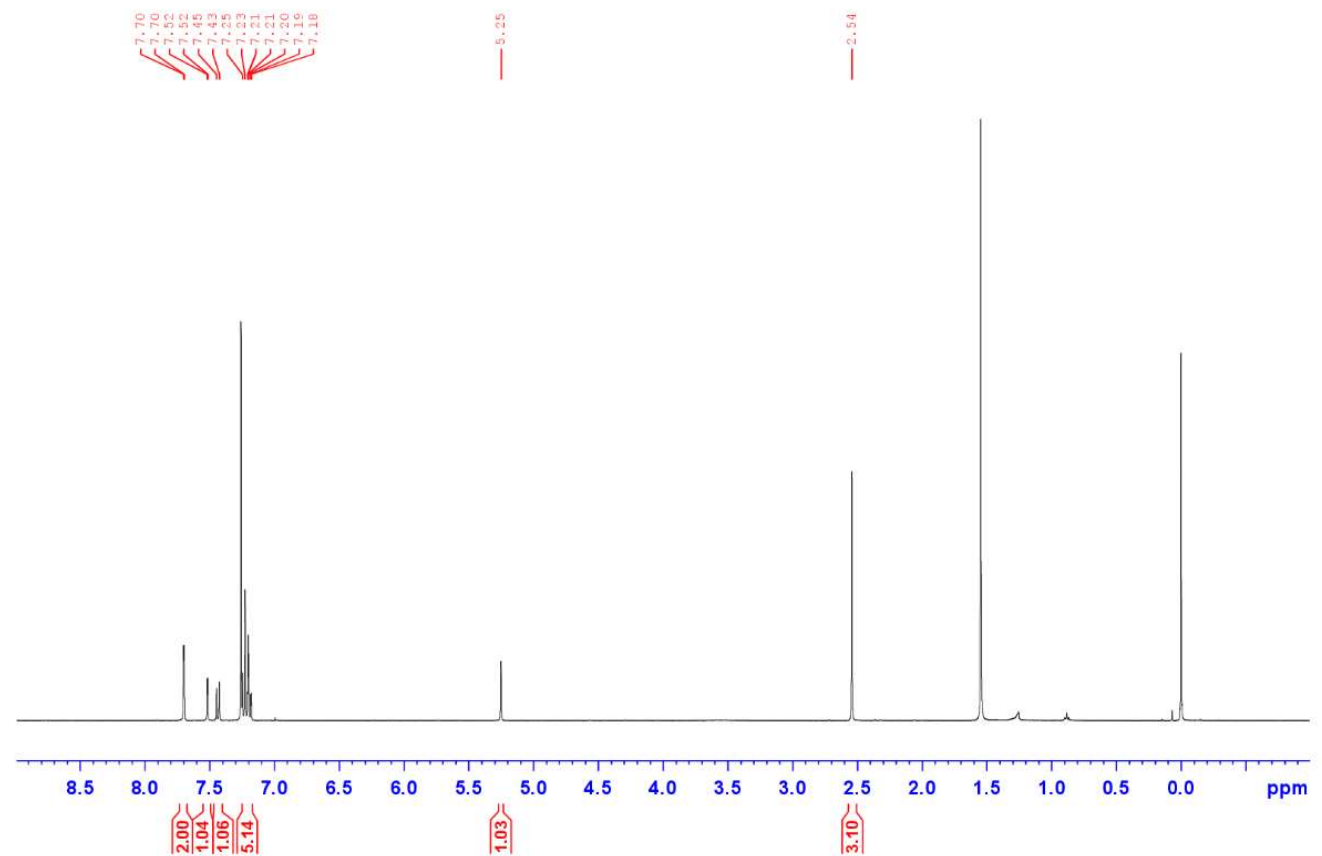

${ }^{1} \mathrm{H}$ NMR (Trip-SMe-Br 3 , minor isomer)

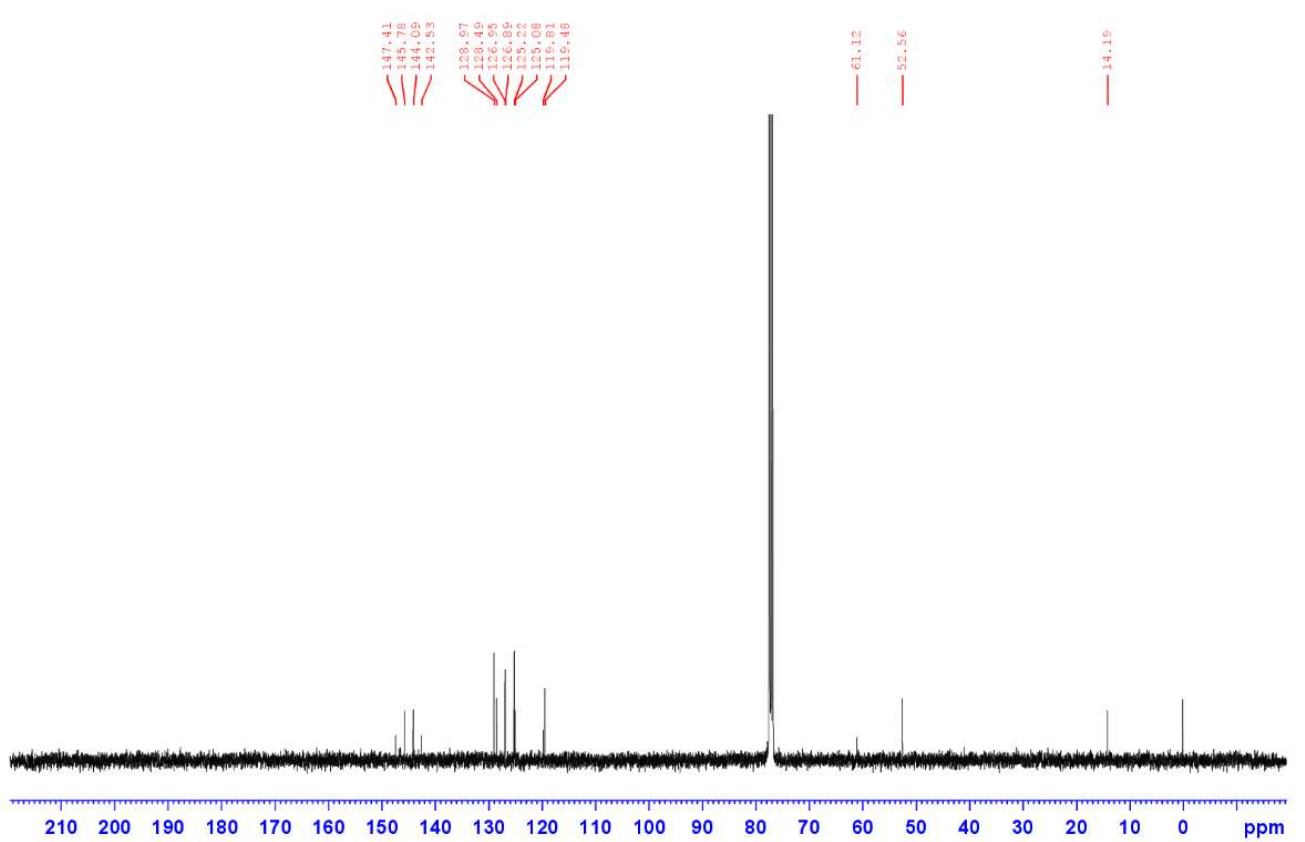




\section{References}

${ }^{1}$ Snyder, S. A.; Treitler, D. S.; Brucks, A. P. Simple Reagents for Direct Halonium-Induced Polyene Cyclizations. J. Am. Chem. Soc. 2010, 132, 14303.

${ }^{2}$ Frisch, M. J.; Trucks, G. W.; Schlegel, H. B.; Scuseria, G. E.; Robb, M. A.; Cheeseman, J. R.; Scalmani, G.; Barone, V.; Petersson, G. A.; Nakatsuji, H.; Li, X.; Caricato, M.; Marenich, A. V.; Bloino, J.; Janesko, B. G.; Gomperts, R.; Mennucci, B.; Hratchian, H. P.; Ortiz, J. V.; Izmaylov, A. F.; Sonnenberg, J. L.; Williams-Young, D.; Ding, F.; Lipparini, F.; Egidi, F.; Goings, J.; Peng, B.; Petrone, A.; Henderson, T.; Ranasinghe, D.; Zakrzewski, V. G.; Gao, J.; Rega, N.; Zheng, G.; Liang, W.; Hada, M.; Ehara, M.; Toyota, K.; Fukuda, R.; Hasegawa, J.; Ishida, M.; Nakajima, T.; Honda, Y.; Kitao, O.; Nakai, H.; Vreven, T.; Throssell, K.; Montgomery, J. A., Jr.; Peralta, J. E.; Ogliaro, F.; Bearpark, M. J.; Heyd, J. J.; Brothers, E. N.; Kudin, K. N.; Staroverov, V. N.; Keith, T. A.; Kobayashi, R.; Normand, J.; Raghavachari, K.; Rendell, A. P.; Burant, J. C.; Iyengar, S. S.; Tomasi, J.; Cossi, M.; Millam, J. M.; Klene, M.; Adamo, C.; Cammi, R.; Ochterski, J. W.; Martin, R. L.; Morokuma, K.; Farkas, O.; Foresman, J. B.; Fox, D. J. Gaussian 16, Revision A.03, Gaussian, Inc., Wallingford CT, 2016.

${ }^{3}$ Chai, J.-D.; Head-Gordon, M. Long-Range Corrected Hybrid Density Functionals with Damped Atom-Atom Dispersion Corrections. Phys. Chem. Chem. Phys. 2008, 10, 6615.

${ }^{4}$ (a) Krishnan, R.; Binkley, J. S.; Seeger, R.; Pople, J. A. Self-Consistent Molecular Orbital Methods. XX. A Basis Set for Correlated Wave Functions. J. Chem. Phys. 1980, 72, 650. (b) McLean, A. D.; Chandler, G. S. Contracted Gaussian Basis Sets for Molecular Calculations. I. Second Row Atoms, Z=11-18. J. Chem. Phys. 1980, 72, 5639 .

5 Tomasi, J.; Mennucci, B.; Cammi, R. Quantum Mechanical Continuum Solvation Models. Chem. Rev. 2005, 105, 2999.

${ }^{6}$ Glendening, E. D.; Badenhoop, J. K.; Reed, A. E.; Carpenter, J. E.; Bohmann, J. A.; Morales, C. M.; Landis, C. R.; Weinhold, F. NBO 6.0, Theor. Chem. Institute, Univ. Wisconsin, Madison 2013.

${ }^{7}$ Murray, D. J.; Patterson, D. D.; Payamyar, P.; Bhola, R.; Song, W.; Lackinger, M.; Schlüter, A. D.; King, B. T. Large Area Synthesis of a Nanoporous Two-Dimensional Polymer at the Air/Water Interface. J. Am. Chem. Soc. 2015, 137, 3450.

${ }^{8}$ Akar, K. B.; Çakmak, O.; Tunç, T. Polybrominated methoxy- and hydroxynaphthalenes. Turk. J. Chem. 2016, $40,332$.

9 Egami, H.; Katsuki, T. Iron-Catalyzed Asymmetric Aerobic Oxidation: Oxidative Coupling of 2-Naphthols. $J$. Am. Chem. Soc. 2009, 131, 6082.

${ }^{10}$ Arun, V.; Reddy, P. O.; Pilania, M; Kumar, D. Ligand- and Base-Free Access to Diverse Biaryls by the Reductive Coupling of Diaryliodonium Salts. Eur. J. Org. Chem. 2016, 2096.

${ }^{11}$ Dahl, B. J.; Mills, N. S. Antiaromaticity in Distal Bisfluorenyl Dications Separated by Multiple Discrete Spacer Units. Org. Lett. 2008, 10, 5605.

12 Zhang, S.; Chen, R.; Yin, J.; Liu, F.; Jiang, H.; Shi, N.; An, Z.; Ma, C.; Liu, B.; Huang, W. Tuning the Optoelectronic Properties of 4,4'- $N, N^{\prime}$-Dicarbazole-biphenyl through Heteroatom Linkage: New Host Materials for Phosphorescent Organic Light-Emitting Diodes. Org. Lett. 2010, 12, 3438.

${ }^{13}$ Korang, J.; Grither, W. R.; McCulla, R. D. Photodeoxygenation of Dibenzothiophene S-Oxide Derivatives in Aqueous Media J. Am. Chem. Soc. 2010, 132, 4466.

${ }^{14}$ Ramakrishna, J.; Venkatakrishnan, P. Bigger and Brighter Fluorenes: Facile $\pi$-Expansion, Brilliant Emission and Sensing of Nitroaromatics. Chem. Asian J. 2017, 12, 181.

15 Reich, H. J.; Cram, D. J. Macro rings. XXXVII. Multiple electrophilic substitution reactions of [2.2]paracyclophanes and interconversions of polysubstituted derivatives. J. Am. Chem. Soc. 1969, 91, 3527.

${ }^{16}$ Lo, C.-Y.; Kumar, M. P.; Chang, H.-K.; Lush, S.-F.; Liu, R.-S. Regioselective Haloaromatization of 1,2- 
Bis(ethynyl)benzene via Halogen Acids and $\mathrm{PtCl}_{2}$. Platinum-Catalyzed 6- $\pi$ Electrocyclization of 1,2-Bis(1'haloethenyl)benzene Intermediates. J. Org. Chem. 2005, 70, 10482.

${ }^{17}$ Kato, S.; Saga, Y.; Kojima, M.; Fuse, H.; Matsunaga, S.; Fukatsu, A.; Kondo, M.; Masaoka, S.; Kanai, M. Hybrid Catalysis Enabling Room-Temperature Hydrogen Gas Release from $N$-Heterocycles and Tetrahydronaphthalenes. J. Am. Chem. Soc. 2017, 139, 2204.

18 Wempe, M. F.; Grunwell, J. R. The Transannular Rearrangement of 5-Cyclodecynone. J. Org. Chem. 1995, 60, 2714.

${ }^{19}$ Fuse, H.; Kojima, M.; Mitsunuma, H.; Kanai, M. Acceptorless Dehydrogenation of Hydrocarbons by NobleMetal-Free Hybrid Catalyst System. Org. Lett. 2018, 20, 2042.

${ }^{20}$ Prebil, R.; Laali, K. K.; Stavber, S. Metal and $\mathrm{H}_{2} \mathrm{O}_{2}$ Free Aerobic Oxidative Aromatic Halogenation with $\left[\mathrm{RNH}_{3}{ }^{+}\right]\left[\mathrm{NO}_{3}{ }^{-}\right] / \mathrm{HX}$ and $\left[\mathrm{BMIM}\left(\mathrm{SO}_{3} \mathrm{H}\right)\right]\left[\left(\mathrm{NO}_{3}\right)_{\mathrm{x}}(\mathrm{X})_{\mathrm{y}}\right](\mathrm{X}=\mathrm{Br}, \mathrm{Cl})$ as Multifunctional Ionic Liquids. Org. Lett. 2013, 15, 2108.

${ }^{21}$ Schmidt, R.; Stolle, A.; Ondruschka, B. Aromatic substitution in ball mills: formation of aryl chlorides and bromides using potassium peroxomonosulfate and NaX. Green Chem. 2012, 14, 1673.

22 Stern, D.; Finkelmeier, N.; Stalke, D. Assessment of the LiX salt-effect in anthracenyl lithiums. Chem. Commun. 2011, 47, 2113.

${ }^{23}$ Rodriguez, R. A.; Pan, C.-M.; Yabe, Y.; Kawamata, Y.; Eastgate, M. D.; Baran, P. S. Palau'chlor: A Practical and Reactive Chlorinating Reagent. J. Am. Chem. Soc. 2014, 136, 6908.

${ }^{24}$ Inamoto, K.; Nozawa, K.; Yonemoto, M.; Kondo, Y. Micellar system in copper-catalysed hydroxylation of arylboronic acids: facile access to phenols. Chem. Commun. 2011, 47, 11775.

${ }^{25}$ Chatterjee, N.; Arfeen, M.; Bharatam, P. V.; Goswami, A. A Metal and Base-Free Chemoselective Primary Amination of Boronic Acids Using Cyanamidyl/Arylcyanamidyl Radical as Aminating Species: Synthesis and Mechanistic Studies by Density Functional Theory. J. Org. Chem. 2016, 81, 5120.

${ }^{26}$ Ueno, A.; Takimoto, M.; O, W. W. N.; Nishiura, M.; Ikariya, T.; Hou, Z. Copper-Catalyzed Formal C-H Carboxylation of Aromatic Compounds with Carbon Dioxide through Arylaluminum Intermediates. Chem. Asian J. 2015, 10, 1010 .

${ }^{27}$ Hou, J.; Li, Z.; Jia, X.-D.; Liu, Z.-Q. Bromination of Arenes Using $\mathrm{I}_{2} \mathrm{O}_{5}-\mathrm{KBr}$ in Water. Synth. Commun. 2014, 44,181

${ }^{28}$ Zhang, L.-S.; Chen, K.; Chen, G.; Li, B.-J. Luo, S.; Guo, Q.-Y.; Wei, J.-B.; Shi, Z.-J. Palladium-Catalyzed Trifluoromethylation of Aromatic C-H Bond Directed by an Acetamino Group. Org. Lett. 2013, 15, 10.

29 Shen, C.; Guo, X.; Yu, J.; Zeng, X.-G.; Peng, L. Zhao, C.-m.; Zhang, F.-L. Synthesis of 2(phenoxymethyl)oxirane derivatives through unexpected rearrangement of oxiran-2-ylmethyl benzenesulfonates. Synth. Commun. 2017, 47, 273.

${ }^{30}$ Sheldrick, G. M. A short history of SHELX. Acta Crystallogr. Sect. A, 2008, 64, 112.

${ }^{31}$ Farrugia, L. J. J. WinGX and ORTEP for Windows: an update. Appl. Cryst., 2012, 45, 849.

32 Burés, J. Variable Time Normalization Analysis: General Graphical Elucidation of Reaction Orders from Concentration Profiles. Angew. Chem., Int. Ed. 2016, 55, 16084. 